\title{
FROM AN ADHESIVE TO A BRITTLE DELAMINATION MODEL IN THERMO-VISCO-ELASTICITY*,**
}

\author{
Riccarda Rossi ${ }^{1}$ and Marita Thomas ${ }^{2}$
}

\begin{abstract}
We address the analysis of a model for brittle delamination of two visco-elastic bodies, bonded along a prescribed surface. The model also encompasses thermal effects in the bulk. The related PDE system for the displacements, the absolute temperature, and the delamination variable has a highly nonlinear character. On the contact surface, it features frictionless Signorini conditions and a nonconvex, brittle constraint acting as a transmission condition for the displacements. We prove the existence of (weak/energetic) solutions to the associated Cauchy problem, by approximating it in two steps with suitably regularized problems. We perform the two consecutive passages to the limit via refined variational convergence techniques.
\end{abstract}

Mathematics Subject Classification. 35K85, 47J20, 49J45, 49S05, 74F07, 74R10.

Received July 10, 2013. Revised November 3, 2013.

Published online October 17, 2014.

\section{INTRODUCTION}

This paper deals with the analysis of a model describing the evolution of brittle delamination between two visco-elastic bodies $\Omega_{+}$and $\Omega_{-}$, bonded along a prescribed contact surface $\Gamma$, see e.g. Figure 1 , over a fixed time interval $(0, T)$. The modeling of delamination follows the approach by Frémond [26,27], which treats this phenomenon within the class of generalized standard materials [36]. More precisely, the adhesiveness of the bonding is modeled with the aid of an internal variable, the so-called delamination variable $z:(0, T) \times \Gamma \rightarrow[0,1]$, which describes the fraction of fully effective molecular links in the bonding. Hence, $z(t, x)=1$ means that the bonding at time $t \in(0, T)$ is fully intact in the material point $x \in \Gamma$, whereas for $z(t, x)=0$ the bonding is completely broken. The weakening of the bonding is a dissipative and unidirectional process, which is assumed

\footnotetext{
Keywords and phrases. Rate-independent evolution of adhesive contact, brittle delamination, Kelvin-Voigt viscoelasticity, nonlinear heat equation, Mosco-convergence, special functions of bounded variation, regularity of sets, lower density estimate.

* R.R. has been partially supported by MIUR-PRIN grants for the projects "Optimal mass transportation, geometric and functional inequalities and applications" and "Calculus of Variations", and by GNAMPA ((Gruppo Nazionale per l'Analisi Matematica, la Probabilità e le loro Applicazioni), of INdAM (Istituto Nazionale di Alta Matematica).

** M.T. has been partially supported by DFG MATHEON project C18 and CRC 910 project A5.

1 DICATAM - Sezione di Matematica, Università di Brescia, via Valotti 9, 25133 Brescia, Italy. riccarda.rossi@unibs.it

2 Weierstrass Institute for Applied Analysis and Stochastics, Mohrenstr. 39, 10117 Berlin, Germany. Thomas@wias-berlin.de
} 


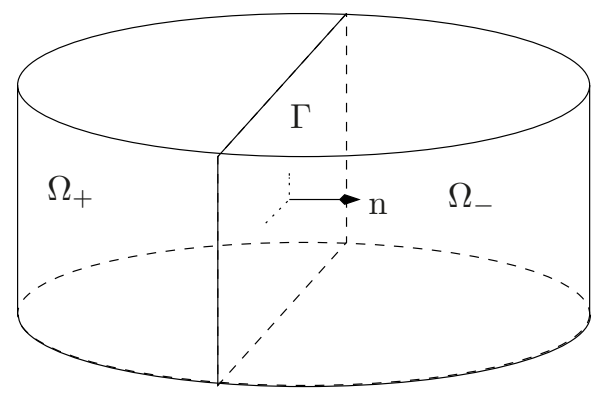

Figure 1. A possible domain $\Omega$ with convex interface $\Gamma$.

to be rate-independent. These facts are modeled by the positively 1-homogeneous dissipation potential

$$
\mathcal{R}_{1}(\dot{z}):=\int_{\Gamma} \mathrm{R}_{1}(\dot{z}) \mathrm{d} \mathcal{H}^{d-1} \quad \text { with } \quad \mathrm{R}_{1}(\dot{z}):=\left\{\begin{array}{l}
a_{1}|\dot{z}| \text { if } \dot{z} \leq 0 \\
+\infty \text { otherwise }
\end{array}\right.
$$

where $\dot{z}$ is the time derivative of $z$ and $\mathcal{H}^{d-1}$ denotes the $(d-1)$-dimensional Hausdorff measure. A further dissipative process is due to viscosity in the bulk, and the amount of dissipated energy is described by the positively 2-homogeneous dissipation potential

$$
\mathcal{R}_{2}(\dot{e}):=\int_{\Omega \backslash \Gamma} \mathrm{R}_{2}(\dot{e}) \mathrm{d} x \quad \text { with } \quad \mathrm{R}_{2}(\dot{e}):=\frac{1}{2} \dot{e}: \mathbb{D}: \dot{e},
$$

acting on the rate of the linearized strain tensor $e$. Here, $\Omega=\Omega_{-} \cup \Gamma \cup \Omega_{+} \subset \mathbb{R}^{d}$ and $\mathbb{D}$ is a positively definite, symmetric fourth-order tensor. In particular, the specific dissipation rate $\mathrm{R}(\dot{e}, \dot{z})=\mathrm{R}_{2}(\dot{e}) \mathrm{d} x+\mathrm{R}_{1}(\dot{z}) \mathrm{d} \mathcal{H}^{d-1}$ is in general a measure which reflects the mixed (i.e., rate-dependent and rate-independent) character of the model. Its absolutely continuous part is given by the (pseudo-)potential of viscous-type dissipative forces in the bulk. The possibly concentrating part, supported on $\Gamma$, features the rate-independent dissipation metric $\mathrm{R}_{1}$.

The visco-elastic response in the bulk material is modeled with the aid of Kelvin-Voigt rheology, neglecting inertia. This rheological model can be described by a parallel arrangement of a linear spring, which instantaneously produces a deformation in proportion to a load, and of a dashpot, which instantaneously produces a velocity in proportion to a load. In other words, in a Kelvin-Voigt visco-elastic solid, a sudden application of a load will not cause an immediate deflection, since it is damped ( $c f$. dashpot arranged in parallel with the spring). Instead, a deformation is built up rather gradually. Hence, the stress tensor of a Kelvin-Voigt visco-elastic solid is of the form $\sigma=\mathbb{C}: e+\mathrm{DR}_{2}(\dot{e})$, where $\mathbb{C}$ is a symmetric, positive definite fourth order tensor and $\mathrm{DR}_{2}$ is the derivative of the viscous dissipation density $\mathrm{R}_{2}$; hereafter, with $\mathrm{D}$ we will denote the Gâteaux derivative. For more details on the rheological modeling of visco-elastic solids the reader is referred to, e.g., [29].

As a further constitutive property of the bulk material it is assumed, that temperature changes cause additional stresses due to thermal expansion. Following [57], for the stress tensor including visco-elastic response and thermal expansion stresses we use the ansatz

$$
\sigma(e, \dot{e}, \theta):=\mathbb{C}: e+\mathrm{DR}_{2}(\dot{e})-\theta \mathbb{C}: \mathbb{E}
$$

with $\theta>0$ the absolute temperature and $\mathbb{E}$ the symmetric matrix of thermal expansion coefficients.

The unknown states in our model are given by the displacement field $u:(0, T) \times\left(\Omega_{-} \cup \Omega_{+}\right) \rightarrow \mathbb{R}^{d}$, the delamination variable $z:(0, T) \times \Gamma \rightarrow[0,1]$, and the absolute temperature $\theta:(0, T) \times\left(\Omega_{-} \cup \Omega_{+}\right) \rightarrow(0, \infty)$. The PDE system describing their evolution consists of the viscous (damped) force balance for $u$, the heat equation for $\theta$ and a flow rule for $z$, which couple the three unknowns in a highly nonlinear manner, see Section 2.1. In the 
analysis, however, we will treat a weak formulation of this PDE system, the so-called energetic formulation. This terminology stems from the fact that this formulation involves the energy and dissipation functionals related to the PDE system.

For the delamination system the overall Helmholtz free energy $\Psi=\Psi(u, z, \theta)$ consists of a bulk and of a surface contribution

$$
\Psi(u, z, \theta)=\Psi^{\mathrm{bulk}}(u, \theta)+\Psi^{\mathrm{surf}}(u, \theta),
$$

where $\Psi^{\text {bulk }}(u, \theta)=\int_{\Omega \backslash \Gamma} \mathrm{W}(e(u), \theta) \mathrm{d} x$ with $\mathrm{W}(e, \theta):=\frac{1}{2} e: \mathbb{C}: e-\theta e: \mathbb{C}: \mathbb{E}-\psi_{0}(\theta)$. Here, $\psi_{0}:(0, \infty) \rightarrow \mathbb{R}$ is a strictly convex function, which is the (purely) thermal part of the free energy. The surface contribution to $\Psi$ does not depend on $\theta$ and indeed coincides with the surface contribution $\Phi^{\text {surf }}$ to the (purely) mechanical part of the energy, the later given by the functional

$$
\Phi(u, z):=\Phi^{\mathrm{bulk}}(u)+\Phi^{\mathrm{surf}}(\llbracket u \rrbracket, z),
$$

where

$$
\Phi^{\text {bulk }}(u)=\int_{\Omega \backslash \Gamma} \frac{1}{2} e: \mathbb{C}: e \mathrm{~d} x \quad \text { and } \quad \Phi^{\text {surf }}(\llbracket u \rrbracket, z)=\int_{\Gamma} \phi^{\text {surf }}(\llbracket u \rrbracket, z) \mathrm{d} \mathcal{H}^{d-1} .
$$

Here, $\llbracket u \rrbracket$ is the jump of $u$ across $\Gamma$. At fixed temperature, for fully rate-independent systems the energetic formulation was developed in $[24,45,47]$, and in this setting it is solely defined by the global stability condition and the global energy balance, i.e. $(u, z):(0, T) \rightarrow \mathcal{Q}$ is an energetic solution of the rate-independent system $\left(\mathcal{Q}, \Phi, \mathcal{R}_{1}\right)$, given by a state space $\mathcal{Q}$, an energy functional $\Phi$ and a dissipation potential $\mathcal{R}_{1}$, if for all $t \in(0, T)$ :

$$
\begin{aligned}
& \forall(\tilde{u}, \tilde{z}) \in \mathcal{Q}: \quad \Phi(t, u(t), z(t)) \leq \Phi(t, \tilde{u}, \tilde{z})+\mathcal{R}_{1}(\tilde{z}-z(t)) \quad \text { (stability), } \\
& \Phi(t, u(t), z(t))+\operatorname{Var}_{\mathcal{R}_{1}}(z ;[0, t])=\Phi(0, u(0), z(0))+\int_{0}^{t} \partial_{t} \Phi(s, u(s), z(s)) \mathrm{d} s \quad \text { (energy balance) }
\end{aligned}
$$

with $\operatorname{Var}_{\mathcal{R}_{1}}(z ;[0, t]):=\sup \sum_{i=1}^{k} \mathcal{R}_{1}\left(z\left(t_{k}\right)-z\left(t_{k-1}\right)\right)$, where the supremum is taken over all partitions of the time interval $[0, t]$. However, conditions (1.6) do not supply a suitable energetic formulation in the temperaturedependent, viscous setting. For this context, an appropriate notion was introduced in [57], see Definition 3.3 ahead. Instead of the two conditions (1.6), the energetic formulation for rate-independent systems with temperature-dependent and viscous effects consists of four conditions: a weak formulation of the momentum balance for $u$, a weak formulation of the heat equation for $\theta$, a so-called semistability condition for $z$, and an energy (in-)equality. The latter two conditions correspond to those in (1.6). In particular, the notion of semistability highlights a significant difference, as, here, stability is only tested for $z$, while $\tilde{u}$ is kept fixed as a solution $u$, i.e.

$$
\forall t \in(0, T) \forall \text { test functions } \tilde{z}: \quad \Phi(t, u(t), z(t)) \leq \Phi(t, u(t), \tilde{z})+\mathcal{R}_{1}(\tilde{z}-z(t)) \quad \text { (semistability). }
$$

The adapted energetic formulation of Definition 3.3 will be analyzed for our delamination model in viscoelastic solids with thermal effects. In particular, we aim at a model for brittle delamination, i.e. it involves the

$$
\text { brittle constraint: } \quad z \llbracket u \rrbracket=0 \quad \text { a.e. on }(0, T) \times \Gamma \text {. }
$$

This condition allows for displacement jumps only in points $x \in \Gamma$, where the bonding is completely broken, i.e. $z(t, x)=0$; in points where $z(t, x)>0$ it ensures $\llbracket u \rrbracket=0$, i.e. the continuity of the displacements. In other words, the brittle constraint (1.8) distinguishes between the crack set, where the displacements may jump, and the complementary set with active bonding, where it imposes a transmission condition on the displacements. Moreover, our model contains a non-penetration constraint ensuring that the two parts of the body, $\Omega_{-}$and 
$\Omega_{+}$, cannot interpenetrate along $\Gamma$ :

$$
\text { non-penetration condition: } \quad \llbracket u \rrbracket \cdot \mathrm{n} \geq 0 \quad \text { a.e. on }(0, T) \times \Gamma \text {. }
$$

Here, $\mathrm{n}$ denotes the unit normal to $\Gamma$ oriented from $\Omega_{+}$to $\Omega_{-}$.

The extremely strict and nonconvex brittle constraint (1.8) causes severe difficulties in the existence analysis, even in the fully rate-independent setting (with fixed temperature and no viscosity), which was addressed in [58]. Therein, the existence of energetic solutions in the sense of (1.6) was not proved directly, but by passing to the limit in a suitable approximation procedure, where (1.8) was replaced by the so-called adhesive contact condition. The latter model involves an energy term which penalizes displacement jumps in points with positive $z$, but does not strictly exclude them, i.e. the

$$
\text { adhesive contact term: } \quad \frac{k}{2} \int_{\Gamma} z|\llbracket u \rrbracket|^{2} \mathrm{~d} \mathcal{H}^{d-1} .
$$

The existence of energetic solutions for the related rate-independent system was proved in [41]. As $k \rightarrow \infty$ it was shown in [58] that the (fully) rate-independent systems of adhesive contact approximate the system for brittle delamination in the sense of $\Gamma$-convergence of rate-independent processes developed in [48].

Our aim is to apply a similar strategy in the viscous, temperature-dependent setting. For this, we want to make use of the results in [54], see also [55], where the existence of energetic solutions in the sense of [57] was proved for adhesive contact in visco-elastic materials with thermal effects. However, as this notion of solution splits the stability test into two separate conditions, weak momentum balance for $u$ and semistability for $z$, we cannot perform the limit passage $k \rightarrow \infty$ in the model from [54] without adding suitable regularization terms. These will allow us to gain additional information on the solutions which, in turn, enables us to construct test functions for the semistability condition and the momentum balance suitably fitted to the properties of the solutions.

We postpone a thorough discussion of these regularization terms to Section 2, where we gain further insight into the PDE system, reveal its analytical difficulties, and explain our results. At this point, let us just mention that our regularizations will consist of a gradient term for $z$ and of a term of $p$-growth in the strain $e$, with $p$ larger than space dimension, ensuring the continuity of the displacements in each of the subdomains $\Omega_{-}$and $\Omega_{+}$. It was proved in [49] that the model for brittle delamination (without a gradient of $z$ ), also treated in [58], describes the evolution of a Griffith-crack along $\Gamma$. This means that $z \in\{0,1\}$, only, and hence $z$ marks the crack set and the unbroken part of $\Gamma$. The fully rate-independent brittle delamination model analyzed in $[49,58,61]$ is thus in accord with the crack models treated in e.g. $[11,15]$, but on a prescribed interface, see also $[39,53]$. In the visco-elastic, temperature-dependent setting we also want to ensure that $z \in\{0,1\}$, and therefore we choose the regularization such that $z$ is the indicator function of a set of finite perimeter in $\Gamma$. As the perimeter is a highly nonconvex term, we first approximate it by a Modica-Mortola term (2.13). Thus, our approximation procedure is the following:

1. from the model for adhesive contact with Modica-Mortola regularization (called Modica-Mortola adhesive contact model) we will pass to the model for adhesive contact with perimeter regularization (called SBVadhesive contact model) in Section 4;

2. from the SBV-adhesive contact model we will then pass in Sections 5 and 6 to the SBV-brittle delamination model (i.e. the model which incorporates the brittle constraint (1.8), but still contains the perimeter term for the delamination variable $z \in\{0,1\}$ ), thus proving the main result of this paper, Theorem 5.2.

Crucial for the passage from adhesive contact to brittle delamination in the visco-elastic, temperature-dependent setting is the construction of suitable test functions for the momentum balance. While referring to the discussion 
at the beginning of Section 5 for all details, let us mention here that such construction requires the continuity of the displacements in $\Omega_{ \pm}$ensured by the regularizing term in the momentum equation, joint with additional information on the semistable delamination variables which solve the adhesive problems. In fact, it involves a fine analysis of their properties, based on tools of geometric measure theory. To such analysis, we have devoted the whole Section 6 . Therein, it will be proved that the finite perimeter sets $Z_{k} \subset \Gamma \subset \mathbb{R}^{d-1}$ underlying the indicator functions $z_{k}$ which are semistable for the adhesive or the brittle problems, satisfy a lower density estimate with respect to the $(d-1)$-dimensional volume, i.e., with respect to the $(d-1)$-dimensional Hausdorff-measure, see (6.6), ensuring that $\mathcal{H}^{d-1}\left(Z_{k} \cap B_{\rho_{\star}}\left(y_{k}\right)\right) \geq \mathfrak{a}(\Gamma) \rho_{\star}^{d-1}$ for all $y_{k} \in \operatorname{supp} z_{k}$ and all $\rho_{\star} \in(0, R)$, with constants $\mathfrak{a}(\Gamma)$ and $R$ depending only on $\Gamma$, the space dimension, and the given data. It is well-known that this type of lower density estimate is satisfied by quasi-minimizers of the perimeter functional, cf. e.g. the monographs [33, 43]. However, these classical works deduce the lower density estimate under the additional assumption that $B_{\rho_{\star}}\left(y_{k}\right) \subset$ $\Gamma$, while we explicitly allow for $B_{\rho_{\star}}\left(y_{k}\right) \backslash \Gamma \neq \emptyset$. Due to this enhancement of the lower density estimate, $\rho_{\star}$ can be kept fixed for all $k \in \mathbb{N}$. We will see that this lower density bound excludes that subsets of $Z_{k}$ concentrate in the null-set of the limit function $z$. Exploiting this property, we will deduce support convergence for the sequence $\left(z_{k}\right)_{k}$, which means that the supports of the delamination variables solving the SBV-adhesive contact problems can be enclosed into balls around the support of the delamination variable for the SBV-brittle delamination model, and the radii of these balls tend to 0 .

This support convergence will be the key property for the aforementioned construction of test functions. In this connection, let us mention that, in contrast to the fully rate-independent case treated in [58], for the limit passage from adhesive to brittle pure $\Gamma$-convergence of the systems in the sense of [48] is no longer sufficient for the present visco-elastic, temperature-dependent systems. Here, Mosco-convergence will be needed, see also [65].

Let us conclude with a few remarks on our reasons for not encompassing inertia in the momentum balance. It is well-known that, already in the frame of adhesive contact systems, the coupling of inertia with Signorini contact conditions poses remarkable analytical problems. In particular, the existence of solutions complying with the energy balance (which plays a crucial role in our analysis) is, to our knowledge, open in the case of bounded domains, see also [54], Remark 5.3 for more comments and references. Indeed, in [54], inertia was included in the momentum equilibrium only upon dealing with special contact conditions for the displacement, which do not encompass Signorini contact. Even in such a context, the passage to the limit in the weak momentum balance from adhesive to brittle would be an open problem. In fact, it would rely on the construction of suitable test functions being in addition sufficiently smooth with respect to time, as required by the weak formulation of the momentum equation with inertia. However, such time regularity seems to be out of reach, as a close perusal of the construction in Section 5.1 shows.

Plan of the paper. After further discussing and motivating our approximation of the brittle delamination model via the SBV-adhesive and the Modica-Mortola adhesive systems in Section 2, in Section 3 we will first collect all the assumptions on the domain and the given data. Hence, we will introduce the energetic formulation of the visco-elastic, temperature-dependent systems for adhesive contact and brittle delamination, and finally discuss the general strategy for proving the existence of energetic solutions. In Section 4 we will carry out the limit passage from Modica-Mortola to SBV-adhesive contact, see Theorem 4.3, in order to obtain an existence result for the SBV-adhesive contact systems (Thm. 4.1). This analysis relies on the existence of energetic solutions to the Modica-Mortola adhesive contact system, stated in Theorem 4.2, which shall be obtained by passing to the limit in a suitable time-discretization scheme in Appendix A.1. These results will be used in order to prove our main result, Theorem 5.2, on the existence of energetic solutions for the SBV-brittle delamination systems. Indeed, in Section 5 we will pass with SBV-adhesive contact to SBV-brittle delamination. As mentioned above, this limit passage bears difficulties in the momentum balance, which can be solved by exploiting additional information on semistable delamination variables, i.e. the lower density estimate and the support convergence. They will be proved in Section 6, by means of tools from geometric measure theory collected for the reader's convenience in Appendix A.2. Finally, in Section 7 we address an alternative scaling for the limit passage from SBV-adhesive to SBV-brittle, which may capture crack initiation in a more concise way. The results therein are a direct consequence of Sections 3-6. 
For the reader's convenience we here collect the symbols used throughout this work. List of symbols

\begin{tabular}{|c|c|}
\hline$u$ & displacement \\
\hline$z$ & delamination parameter \\
\hline$\theta$ & absolute temperature \\
\hline$w$ & enthalpy \\
\hline$e$ & linearized strain tensor \\
\hline$\sigma$ & stress tensor \\
\hline$\llbracket u \rrbracket$ & jump of $u$ across contact surface $\Gamma$ \\
\hline $\mathbb{C}$ & elasticity tensor \\
\hline $\mathbb{D}$ & viscosity tensor \\
\hline $\mathbb{E}$ & thermal expansion coefficients \\
\hline \multicolumn{2}{|l|}{$\mathbb{B}=\mathbb{C}: \mathbb{E}$} \\
\hline$F$ & applied bulk force \\
\hline$f$ & applied traction \\
\hline$c_{\mathrm{V}}$ & heat capacity \\
\hline $\mathbb{K}$ & heat conduction coefficients \\
\hline$H$ & bulk heat source \\
\hline$h$ & heat source on $\partial \Omega$ \\
\hline$a_{0}\left(a_{1}\right)$ & spec. en. stored (dissip.) by delam. \\
\hline$\eta$ & heat-transfer coefficient \\
\hline$\Phi$ & mechanical energy, (1.4) \\
\hline$\Phi^{\mathrm{bulk}}$ & bulk mechanical energy, (3.15) \\
\hline $\begin{array}{l}\mathrm{W}_{p} \\
\Phi^{\text {surf }}\end{array}$ & $\begin{array}{l}\text { elastic energy density of } p \text {-growth, (3.15) } \\
\text { mechanical surface energy, (1.4) }\end{array}$ \\
\hline$\Phi_{k, m}^{\text {surf }}$ & surface energy for Modica-Mortola system, (3.17) \\
\hline$\Phi_{k}^{\text {surf }}$ & surface energy for SBV-adhesive syst., (3.19) \\
\hline$\Phi_{\mathrm{b}}^{\text {surf }}$ & surface energy for SBV-brittle syst., (3.22) \\
\hline $\mathcal{G}_{m}$ & Modica-Mortola regularization in $\Phi_{k, m}^{\text {surf }},(2.13)$ \\
\hline $\mathcal{G}_{\mathrm{b}}$ & gradient term for $\Phi_{k}^{\text {surf }}$ and $\Phi_{\mathrm{b}}^{\text {surf }},(2.9)$ \\
\hline$J_{k}$ & adhesive contact energy density, (3.17) \\
\hline$J_{\infty}$ & brittle constraint, $(2.4)$ \\
\hline $\mathcal{R}_{1}$ & rate-independent dissipation potential, (1.1a) \\
\hline $\operatorname{Var}_{\mathcal{R}_{1}}$ & $\mathcal{R}_{1}$-total variation, $(3.32)$ \\
\hline$\xi_{\dot{z}}^{\text {surf }}$ & measure-valued time-derivative of $z,(3.34)$ \\
\hline $\mathcal{R}_{2}$ & viscous dissipation potential, (1.1b) \\
\hline $\mathcal{U}$ & state space for $u$ (M.-M. and SBV-adh. syst.), (3.24) \\
\hline $\mathcal{U}_{z}$ & state space for $u$ (SBV-brittle syst.), (3.25) \\
\hline $\mathcal{Z}_{\mathrm{MM}}$ & state space for $z$ (M.-M. system), (3.18) \\
\hline $\mathcal{Z}_{\mathrm{SBV}}$ & admiss. set for $z$ (adh. and brittle SBV-syst.), (3.21) \\
\hline $\mathcal{W}$ & space of test functions for enthalpy equation (3.26) \\
\hline
\end{tabular}

\section{Presentation of the models and analytical Difficulties}

In this section, we first detail the classical formulation of the PDE system describing the brittle delamination model for visco-elastic materials with thermal effects. We then highlight the main difficulties related to its analysis and motivate its approximation by the SBV- and Modica-Mortola adhesive systems. 


\subsection{The classical formulation of the problem}

Throughout the paper we assume that $\Omega \subset \mathbb{R}^{d}, d \geq 2$, is a bounded domain with $\Omega=\Omega_{+} \cup \Gamma \cup \Omega_{-}$and $\Gamma$ representing the prescribed (flat, convex) interface with possible delamination, see Figure 1 . We denote by $\mathrm{n}$ both the outward unit normal to $\partial \Omega$, and the unit normal to $\Gamma$ oriented from $\Omega_{+}$to $\Omega_{-}$. Given $v \in W^{1,2}\left(\Omega \backslash \Gamma ; \mathbb{R}^{d}\right)$, with $v^{+}\left(v^{-}\right)$we signify the restriction of $v$ to $\Omega_{+}\left(\Omega_{-}\right)$. We denote by

$$
\llbracket v \rrbracket:=\left.v^{+}\right|_{\Gamma}-\left.v^{-}\right|_{\Gamma} \text { the jump of } v \text { across } \Gamma .
$$

The PDE system, coupling the momentum equation in the bulk (2.2a) for the displacement $u$, the heat equation $(2.2 \mathrm{~b})$ for the absolute temperature $\theta$, and the evolution $(2.2 \mathrm{k})-(2.2 \mathrm{n})$ for the delamination parameter $z$, formally reads:

$$
\begin{array}{ll}
-\operatorname{div} \sigma(u, \dot{u}, \theta)=F & \text { in }(0, T) \times(\Omega \backslash \Gamma), \\
c_{\mathrm{v}}(\theta) \dot{\theta}-\operatorname{div}(\mathbb{K}(e(u), \theta) \nabla \theta)=e(\dot{u}): \mathbb{D}: e(\dot{u})-\theta \mathbb{E}: \mathbb{C}: e(\dot{u})+H & \text { in }(0, T) \times(\Omega \backslash \Gamma), \\
u=0 & \text { on }(0, T) \times \Gamma_{\mathrm{D}}, \\
\left.\sigma(u, \dot{u}, \theta)\right|_{\Gamma_{\mathrm{N}}} \mathrm{n}=f & \text { on }(0, T) \times \Gamma_{\mathrm{N}}, \\
(\mathbb{K}(e(u), \theta) \nabla \theta) \mathrm{n}=h & \text { on }(0, T) \times \partial \Omega, \\
\llbracket \sigma \rrbracket \mathrm{n}=0 & \text { on }(0, T) \times \Gamma, \\
\llbracket u \rrbracket \cdot \mathrm{n} \geq 0 & \text { on }(0, T) \times \Gamma, \\
\left.\sigma(u, \dot{u}, \theta)\right|_{\Gamma} \mathrm{n} \cdot \mathrm{n} \geq 0 \quad \text { wherever } z(\cdot)=0 & \text { on }(0, T) \times \Gamma, \\
\left.\sigma(u, \dot{u}, \theta)\right|_{\Gamma} \mathrm{n} \cdot \llbracket u \rrbracket=0 & \text { on }(0, T) \times \Gamma, \\
z \llbracket u \rrbracket=0 & \text { on }(0, T) \times \Gamma, \\
\dot{z} \leq 0 & \text { on }(0, T) \times \Gamma, \\
\xi \leq a_{1}+a_{0} & \text { on }(0, T) \times \Gamma, \\
\dot{z}\left(\xi-a_{0}-a_{1}\right)=0 & \text { on }(0, T) \times \Gamma, \\
\xi \in \partial_{z} \Phi(u, z) & \text { on }(0, T) \times \Gamma, \\
\frac{1}{2}\left(\left.\mathbb{K}(e(u), \theta) \nabla \theta\right|_{\Gamma} ^{+}+\left.\mathbb{K}(e(u), \theta) \nabla \theta\right|_{\Gamma} ^{-}\right) \cdot \mathrm{n}+\eta(\llbracket u \rrbracket, z) \llbracket \theta \rrbracket=0 & \text { on }(0, T) \times \Gamma, \\
\llbracket \mathbb{K}(e(u), \theta) \nabla \theta \rrbracket \cdot \mathrm{n}=-a_{1} \dot{z} & \text { on }(0, T) \times \Gamma,
\end{array}
$$

where $\partial \Omega=\Gamma_{\mathrm{D}} \cup \Gamma_{\mathrm{N}}$ with $\Gamma_{\mathrm{D}}$ the Dirichlet and $\Gamma_{\mathrm{N}}$ the Neumann parts of the boundary $\partial \Omega$.

System (2.2) was derived in [54], Section 2 starting from the Helmholtz free energy (1.3) and the dissipation potentials (1.1); its thermodynamical consistency was shown, in the sense that the Clausius-Duhem inequality and the positivity of temperature are satisfied. In the following lines, we will confine ourselves to just explaining the meaning of the equations; for more details we refer to [54].

In $(2.2 \mathrm{a}),(2.2 \mathrm{~d}),(2.2 \mathrm{f}),(2.2 \mathrm{~h})$, and $(2.2 \mathrm{i})$, the term $\sigma=\sigma(u, v, \theta):=\mathbb{D}: e(v)+\mathbb{C}:(e(u)-\mathbb{E} \theta)$ is the stress tensor, which encompasses Kelvin-Voigt rheology and thermal expansion, as explained along with (1.2). Here, the tensors

$$
\mathbb{C}, \mathbb{D}: \mathbb{R}_{\mathrm{sym}}^{d \times d} \rightarrow \mathbb{R}_{\mathrm{sym}}^{d \times d} \text { are of } 4 \text { th-order, positive definite, symmetric, } \operatorname{div}(\mathbb{C}: e(u)) \text { has a potential, }
$$

in particular, $\mathbb{C}_{i j k l}=\mathbb{C}_{j i k l}=\mathbb{C}_{k l i j}$, and the same for $\mathbb{D} ; \mathbb{E} \in \mathbb{R}^{d \times d}$ is a matrix of thermal-expansion coefficients. Moreover, $F:(0, T) \times \Omega \rightarrow \mathbb{R}^{d}$ in $(2.2 \mathrm{a})$ is the applied bulk force, $f:(0, T) \times \Gamma_{\mathrm{N}} \rightarrow \mathbb{R}^{d}$ in $(2.2 \mathrm{~d})$ is the applied traction, while $H:(0, T) \times \Omega \rightarrow \mathbb{R}$ in $(2.2 \mathrm{~b})$ and $h:(0, T) \times \partial \Omega \rightarrow \mathbb{R}$ in $(2.2 \mathrm{e})$ are external heat sources.

In the heat equation $(2.2 \mathrm{~b})$, the function $c_{\mathrm{V}}:(0,+\infty) \rightarrow(0,+\infty)$ is the heat capacity of the system, defined from the thermal energy $\psi_{0}$ by $c_{\mathrm{v}}(\theta)=\theta \psi_{0}{ }^{\prime \prime}(\theta)$. Moreover, $-\mathbb{K}(e, \theta) \nabla \theta$ determines the heat flux according to Fourier's law, with $\mathbb{K}=\mathbb{K}(e, \theta)$ as the positive definite matrix of heat conduction coefficients. The terms 
$e(\dot{u}): \mathrm{DR}_{2}(e(\dot{u}))=e(\dot{u}): \mathbb{D}: e(\dot{u})$ and $-\theta \mathbb{E}: \mathbb{C}: e(\dot{u})$ on the right-hand side of $(2.2 \mathrm{~b})$ are heat sources due to viscous and thermal expansion stresses, and they generate a coupling between the heat and the momentum equation.

Further, (2.2c) and (2.2d) are the Dirichlet and Neumann conditions for $u$ and (2.2e) is the Neumann condition for the heat flux across the boundary of $\Omega$; on the contact surface $\Gamma$ we have the transmission condition $(2.2 \mathrm{f})$ and conditions (2.2g)-(2.2i). The latter yield the complementarity form of the Signorini contact conditions, preventing penetration of either of the bodies $\Omega_{+}$and $\Omega_{-}$along the interface. Furthermore, $(2.2 \mathrm{j})$ is the brittle constraint, which can be interpreted as a transmission condition on the contact surface $\Gamma$, as explained along with (1.8).

The complementarity conditions $(2.2 \mathrm{k})-(2.2 \mathrm{n})$ determine the evolution of the delamination variable. Observe that they rewrite as $\partial I_{(-\infty, 0]}(\dot{z})+\xi-a_{0}-a_{1} \ni 0$, with $\xi \in \partial_{z} \Phi(u, z)$. Now, (2.2k) ensures the unidirectionality of the delamination process, as crack healing is prevented. In $(2.21),(2.2 \mathrm{~m})$, the coefficient $a_{0}$ (resp. $\left.a_{1}\right)$ is the phenomenological specific energy per area which is stored (resp. dissipated) by disintegrating the adhesive. The overall activation energy to trigger the debonding process in the adhesive is then $a_{0}+a_{1}$. Moreover, in $(2.2 \mathrm{n})$, $\partial_{z} \Phi(u, z)$ denotes the (convex analysis) subdifferential of the mechanical energy $\Phi$ introduced in (1.4) and (1.5). Hereby, the surface part of the energy has the density $\phi^{\operatorname{surf}}(\llbracket u \rrbracket, z):=I_{\llbracket \llbracket u \cdot \cdot \mathrm{n} \geq 0]}(u)+I_{[0,1]}(z)+J_{\infty}(\llbracket u \rrbracket, z)-a_{0} z$, where $I_{[\llbracket u \rrbracket \cdot \mathrm{n} \geq 0]}(u)$ stands for the indicator function of the non-penetration condition, i.e. $I_{[\llbracket u \rrbracket \cdot \mathrm{n} \geq 0]}(u)=0$ if $\llbracket u \rrbracket \cdot \mathrm{n} \geq 0$ and $I_{[\llbracket u \rrbracket \cdot \mathrm{n} \geq 0]}(u)=\infty$ otherwise. Moreover, $I_{[0,1]}$ denotes the indicator function of the interval $[0,1]$, i.e $I_{[0,1]}(r)=0$ if $r \in[0,1]$ and $I_{[0,1]}(r)=+\infty$ otherwise. The third operator refers to the indicator function featuring the brittle constraint

$$
J_{\infty}(v, z)=I_{\{v z=0\}}(v, z), \text { i.e. } J_{\infty}(v, z)=\left\{\begin{array}{lr}
0 & \text { if } v z=0 \\
+\infty & \text { otherwise }
\end{array}\right.
$$

Finally, conditions (2.2o) and (2.2p) balance the heat transfer across $\Gamma$ with the ongoing crack growth. In particular, the function $\eta$ in the boundary condition (2.2o) on $\Gamma$ for $\theta$ is a heat-transfer coefficient, determining the heat convection through $\Gamma$, which depends on the state of the bonding and on the distance between the crack lips. We refer to [54], Remark 3.3 for further details.

\subsection{Regularization and approximation via adhesive contact models}

The analysis of system (2.2) encounters several difficulties: first of all, the mixed character of the problem, coupling rate-independent evolution for $z$, with rate-dependent equations for $u$ and $\theta$. Let us also mention the highly nonlinear character of the heat equation, with a quadratic term on the right-hand side. The evolution of $z$ is ruled by the complementarity conditions $(2.2 \mathrm{k})-(2.2 \mathrm{n})$, which can be reformulated as the subdifferential inclusion

$$
\partial I_{(-\infty, 0]}(\dot{z}(t, x))+\partial_{z} \Phi(u(t, x), z(t, x))-a_{0}-a_{1} \ni 0, \quad(t, x) \in(0, T) \times \Gamma .
$$

Let us observe that the subdifferential inclusion (2.5) for $z$ is effectively triply nonlinear, featuring three multivalued operators, since $\partial_{z} \Phi(u, z)$ involves the subdifferentials of both $I_{[0,1]}$ and $J_{\infty}$. Here, an additional difficulty

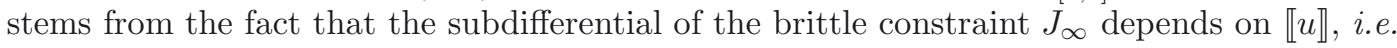

$$
\partial_{z} J_{\infty}(\llbracket u \rrbracket, z)= \begin{cases}\emptyset & \text { if } z \neq 0 \text { and } \llbracket u \rrbracket \neq 0 \\ 0 & \text { if } \llbracket u \rrbracket=0, \\ \mathbb{R} & \text { if } \llbracket u \rrbracket \neq 0 \text { and } z=0\end{cases}
$$

and this dependence is of course transferred to $\partial_{z} \Phi(u, z)$.

Nonetheless, it is the analysis of the boundary value problem for the momentum equation which brings along the most challenging problems. Indeed, in view of $(2.2 \mathrm{~g})-(2.2 \mathrm{j})$, on the contact surface $\Gamma$ we have for the displacement $u$ a double constraint, namely the non-penetration $\llbracket u \rrbracket \cdot \mathrm{n} \geq 0$, and the nonconvex brittle constraint $z \llbracket u \rrbracket=0$. Such constraints are reflected in the variational formulation of the boundary value problem for $(2.2 \mathrm{a})$ 
as a variational inequality, i.e.

$$
\begin{gathered}
\llbracket u \rrbracket \cdot \mathrm{n} \geq 0, \quad z \llbracket u \rrbracket=0 \text { on }(0, T) \times \Gamma, \text { and } \\
\int_{\Omega \backslash \Gamma}(\mathbb{D}: e(\dot{u})+\mathbb{C}:(e(u)-\mathbb{E} \theta)): e(v-u) \mathrm{d} x \geq \int_{\Omega} F \cdot(v-u) \mathrm{d} x+\int_{\Gamma_{\mathrm{N}}} f \cdot(v-u) \mathrm{d} x
\end{gathered}
$$

for all test functions $v$ with suitable regularity and such that $\llbracket v \rrbracket \cdot \mathrm{n} \geq 0$ and $z \llbracket v \rrbracket=0$ a.e. on $(0, T) \times \Gamma$. A major difficulty is that the brittle constraint involves $z$, and accordingly the set of test functions in $(2.7)$ depends on $z$.

The SBV-brittle delamination system. To handle the coupling of the brittle and of the non-penetration constraints, we will approximate system $(2.2)$ by penalizing the condition $z \llbracket u \rrbracket=0$ on $(0, T) \times \Gamma$. For the passage to the limit in the weak formulation of the momentum equation, a suitable construction of approximate test functions will be needed. This construction relies on a higher spatial regularity for the displacement variable $u$. Therefore, we have to regularize the momentum equation (2.2a) by means of a tensorial $p$-Laplacian term, with $p>d$. More precisely, in the momentum balance (2.2a) and in the boundary conditions $(2.2 \mathrm{~d}),(2.2 \mathrm{f}),(2.2 \mathrm{~h})$, and (2.2i), from now on the stress tensor $\sigma$ will be given by

$$
\sigma=\sigma(u, v, \theta):=\mathbb{D}: e(v)+\mathbb{C}:(e(u)-\mathbb{E} \theta)+|e(u)|^{p-2} \mathbb{H}: e(u) \quad \text { with } p>d
$$

and $\mathbb{H}$ a fourth-order symmetric positive-definite tensor. Note that the term $|e(u)|^{p-2} \mathbb{H}: e(u)$ ensures that $u \in$ $W^{1, p}\left(\Omega_{ \pm}\right) \subset \mathrm{C}^{0}\left(\overline{\Omega_{ \pm}}\right)$(since $p>d$ ), which is crucial for tackling the brittle constraint $z \llbracket u \rrbracket=0$. Materials with constitutive laws of $p$-Laplacian-type, also known as power-law materials, are used in literature in order to model strain hardening or softening $[37,40]$. In particular, the case of power $p$ larger than space dimension is used to describe strain hardening, also at small strains [6].

Furthermore, we shall also regularize the delamination variable $z$ through an additional gradient term $\mathcal{G}(z)$. Gradient regularizations of the type $\mathcal{G}(z)=\int_{\Omega} \frac{1}{r}|\nabla z|^{r} \mathrm{~d} x$ are widely used and accepted in models for volume damage (see e.g. [10,28,46,49,61,64]), but also in models for delamination and adhesive contact $[1,8,9,25,27]$. In particular, the latter works involve the gradients of $z \in H^{1}(\Gamma)$, while here, we reduce the regularization to BV-type. Because of this, the delamination variable may jump in space and therefore drop instantaneously from one value to another. Let us stress that this brings our model closer to describing the physics of cracking.

To be more precise, we take the state space $\mathcal{Z}$ for $z$ as a subset of the space $\operatorname{BV}(\Gamma)$ of functions of bounded variation on $\Gamma$, whose distributional gradient is a finite Radon measure on $\Gamma$. Hence, we consider $\mathcal{G}_{\mathrm{b}}(z)=$ $\mathrm{b}|\mathrm{D} z|(\Gamma)$ for some $\mathrm{b}>0$, where $|\mathrm{D} z|(\Gamma)$ denotes the variation of the measure $\mathrm{D} z$ in $\Gamma$. Moreover, we add a further constraint in our delamination system, namely that the variable $z$ only takes the values $\{0,1\}$. Therefore, our model accounts for just two states of the bonding between $\Omega_{+}$and $\Omega_{-}$, that is, fully effective and completely ineffective. On the one hand, the feature that $z \in\{0,1\}$ makes our model akin to a Griffith-type model for crack evolution (along a prescribed interface). Therein, the delamination variable $z$ individuates the crack set, and thus only takes either the value 0 , or 1 , see $[49,61]$. On the other hand, such a restriction brings along some analytical advantages, as the considerations in Section 6 will show later on. Since $z \in\{0,1\}, z$ can be viewed as the characteristic function of a set $Z$ with finite perimeter. Therefore, the gradient term $\mathcal{G}_{\mathrm{b}}$ reduces to

$$
\mathcal{G}_{\mathrm{b}}(z)=\mathrm{b}|\mathrm{D} z|(\Gamma)=\mathrm{b} P(Z, \Gamma),
$$

where $P(Z, \Gamma)$ is the perimeter of the set $Z$ in $\Gamma$, cf. Definition A.6. We will also use that $\mathcal{G}_{\mathrm{b}}(z)=\mathcal{H}^{d-2}\left(\mathrm{~J}_{z}\right)$, where $\mathcal{H}^{d-2}$ denotes the $(d-2)$-dimensional Hausdorff measure and $\mathrm{J}_{z}$ is the jump set of $z \in \operatorname{SBV}(\Gamma ;\{0,1\})$, see Definition A.15. Here, $\operatorname{SBV}(\Gamma ;\{0,1\})$ is the set of characteristic functions of subsets of $\Gamma$ with finite perimeter. In particular, the acronym SBV stands for special functions of bounded variation, which is the subspace of BV of functions whose total variation has no Cantor part, see [4] for more details. With the regularization $\mathcal{G}_{\mathrm{b}}$ given by (2.9), the subdifferential inclusion (2.5) is formally replaced by

$$
\partial I_{(-\infty, 0]}(\dot{z}(t, x))+\partial_{z} \Phi(u(t, x), z(t, x))+\partial \mathcal{G}_{\mathrm{b}}(z(t, x))-a_{0}-a_{1} \ni 0,
$$

for a.a. $(t, x) \in(0, T) \times \Gamma$. In fact, we will analyze a weak formulation of (2.10). 
Throughout the paper, we shall refer to the PDE system (2.2), with (2.5) replaced by (2.10), and the stress $\sigma$ given by (2.8), as the SBV-brittle delamination system. We shall propose a suitable notion of weak solution for this system, $c f$. Definition 3.9 of energetic solution. This solution concept consists of the weak formulations of the boundary-value problems for the momentum equation (2.2a), with $\sigma$ from (2.8), and for the heat equation (2.2b), as well as of a semistability condition in place of (2.10), and of an energy (in-)equality. Our main Theorem 5.2 states the existence of energetic solutions to the SBV-brittle delamination system. In what follows, we hint at the strategy for the proof of this existence result, and in doing so we motivate the two aforementioned gradient regularizations.

The SBV-adhesive contact system. In order to deal with the brittle constraint $z \llbracket u \rrbracket=0$ on $(0, T) \times \Gamma$, we approximate problem (2.2), with an adhesive contact problem, where $(2.2 \mathrm{~g})-(2.2 \mathrm{i})$ are replaced by

$$
\left.\begin{array}{l}
\llbracket u(t, x) \rrbracket \cdot \mathrm{n} \geq 0 \\
\left(\left.\sigma(u(t, x), \dot{u}(t, x), \theta(t, x))\right|_{\Gamma} \mathrm{n}+k z(t, x) \llbracket u(t, x) \rrbracket\right) \cdot \mathrm{n} \geq 0 \\
\left(\left.\sigma(u(t, x), \dot{u}(t, x), \theta(t, x))\right|_{\Gamma} \mathrm{n}+k z(t, x) \llbracket u(t, x) \rrbracket\right) \cdot \llbracket u(t, x) \rrbracket=0
\end{array}\right\}
$$

for a.a. $(t, x) \in(0, T) \times \Gamma$, whereas instead of $(2.10)$ we have

$$
\partial I_{(-\infty, 0]}(\dot{z}(t, x))+\partial I_{[0,1]}(z(t, x))+\frac{1}{2} k|\llbracket u(t, x) \rrbracket|^{2}+\partial \mathcal{G}_{\mathrm{b}}(z(t, x))-a_{0}-a_{1} \ni 0, \quad(t, x) \in(0, T) \times \Gamma,
$$

with $k>0$ a fixed constant. Formally, (2.5), along with the brittle constraint $z \llbracket u \rrbracket=0$ on $(0, T) \times \Gamma$, arises in the limit as $k \rightarrow \infty$ of (2.11) and (2.12). We shall refer to the approximate problem obtained replacing $(2.2 \mathrm{~g})-(2.2 \mathrm{j})$ and (2.10), with (2.11) and (2.12), respectively (combined with the quasi-static momentum equation (2.2a) with $\sigma$ from (2.8)), as the SBV-adhesive contact system. First, we shall prove existence of energetic solutions for the related Cauchy problem in Theorem 4.1. Hence we shall take the limit as $k \rightarrow \infty$ : Theorem 5.2 states that, up to a subsequence, solutions to the SBV-adhesive contact systems converge to solutions of the SBV-brittle delamination system.

The Modica-Mortola adhesive contact system. Since the SBV-gradient term in (2.12) is highly nonconvex, to prove existence for the (weak formulation of the) SBV-adhesive system we use a Modica-Mortola type approximation. This kind of regularization has been well-known in the mathematical literature for more than thirty years. Indeed, in the papers [50,51] (see also [2]), within phase transition modeling it was proved that the so-called static Modica-Mortola functional $\Gamma$-converges to the static perimeter functional. Modica-Mortola approximations in the context of models for volume damage have also been exploited in [31,62]. The Modica-Mortola functional is

$$
\mathcal{G}_{m}(z):=\int_{\Gamma}\left(\frac{m}{2} g(z)+\frac{1}{2 m}|\nabla z|^{2}+I_{[0,1]}(z)\right) \mathrm{d} \mathcal{H}^{d-1} \quad \text { with } g(z)=z^{2}(1-z)^{2} \text { and } m>0 .
$$

Accordingly, we will approximate the SBV-adhesive system by replacing the subdifferential inclusion (2.12) for $z$, with

$$
\partial I_{(-\infty, 0]}(\dot{z}(t, x))+\partial I_{[0,1]}(z(t, x))+\frac{1}{2} k|\llbracket u(t, x) \rrbracket|^{2}+\frac{m}{2} g^{\prime}(z(t, x))-\frac{1}{m} \Delta z(t, x)-a_{0}-a_{1} \ni 0,
$$

for a.a. $(t, x) \in(0, T) \times \Gamma$. The resulting approximate problem will be called Modica-Mortola adhesive contact system. Since the existence result from [54] does not apply to this system, we will prove the existence of solutions in Theorem 4.2. Observe that the $p$-regularizing term in (2.8) is not needed for the related analysis, as it only plays a role in the passage to the brittle limit. However we will keep it in the Modica-Mortola system as well, for consistency of exposition. 


\section{General Setup AND Weak Formulation}

In this section we present a suitable notion of weak formulation for the visco-elastic, temperature-dependent systems of adhesive contact and brittle delamination, i.e. the energetic formulation developed in [57]. Prior to establishing this formulation in Section 3.3, in Section 3.1 we perform the so-called enthalpy reformulation of system (2.2) (and its regularizations), following [57]. Then, in Section 3.5 the general strategy of the existence proof will be outlined. Although Definition 3.3 of energetic solution does not rely on a specific set of assumptions on the geometrical setting and the problem data, subsequent results such as Theorem 3.10 do. That is why, we have chosen to preliminarily collect all of the assumptions on the given data in Section 3.2, appropriate for all the systems and all the limit passages. Let us now fix some general notation.

Notation 3.1 (Function spaces).

Throughout the paper, for $p \in(1, \infty)$ we shall adopt the notation

$$
W_{\Gamma_{\mathrm{D}}}^{1, p}\left(\Omega \backslash \Gamma ; \mathbb{R}^{d}\right)=\left\{v \in W^{1, p}\left(\Omega \backslash \Gamma ; \mathbb{R}^{d}\right): \quad v=0 \text { on } \Gamma_{\mathrm{D}}\right\} .
$$

We recall that

$$
\left.u \mapsto u\right|_{\Gamma}: W^{1, p}(\Omega \backslash \Gamma) \rightarrow W^{1,1-\frac{1}{p}}(\Gamma) \text { continuously }
$$

with $\Gamma=\partial \Omega$, or $\Gamma=\Gamma$, or $\Gamma=\Gamma_{\mathrm{N}}$. Furthermore, we shall exploit that, for $p>d$, the following embedding holds for $W^{1, p}\left(\Omega_{ \pm}\right)$(and obviously for the Sobolev space $W^{1, p}\left(\Omega_{ \pm} ; \mathbb{R}^{d}\right)$ of vector-valued functions)

$$
W^{1, p}\left(\Omega_{ \pm}\right) \subset \mathrm{C}^{0}\left(\overline{\Omega_{ \pm}}\right) \text {compactly. }
$$

We shall denote by $\langle\cdot, \cdot\rangle$ the duality pairing between the spaces $W^{1, q}\left(\Omega \backslash \Gamma ; \mathbb{R}^{d}\right)^{*}$ and $W^{1, q}\left(\Omega \backslash \Gamma ; \mathbb{R}^{d}\right)$, and between $W^{1, q}(\Omega \backslash \Gamma)^{*}$ and $W^{1, q}(\Omega \backslash \Gamma)$, for any $1 \leq q<\infty$.

For a (separable) Banach space $X$, we shall use the notation $\mathrm{BV}([0, T] ; X)$ for the space of functions from $[0, T]$ with values in $X$ that have bounded variation on $[0, T]$. Notice that all these functions are defined everywhere on $[0, T]$.

Finally, throughout the paper we will use the symbols $c, c^{\prime}, C$, and $C^{\prime}$, for various positive constants depending only on known quantities.

\subsection{Enthalpy reformulation}

Following [54,57], we shall in fact analyze a reformulation of the PDE system (2.2), in which we replace the heat equation $(2.2 \mathrm{~b})$ with an enthalpy equation, $c f$. system (3.6) below. This is motivated by the fact that the nonlinear term $c_{\mathrm{v}}(\theta) \dot{\theta}$ makes it difficult to implement a time-discretization scheme for $(2.2 \mathrm{~b})$. In turn, timediscretization will provide the basic existence result for the Modica-Mortola adhesive contact system. Therefore, as in $[54,57]$ we are going to resort to a change of variables for $\theta$, by means of which $c_{\mathrm{v}}(\theta) \dot{\theta}$ is replaced by the linear contribution $\dot{w}$.

Hereafter, we switch from the absolute temperature $\theta$, to the enthalpy $w$, defined via the so-called enthalpy transformation, i.e.

$$
w=h(\theta):=\int_{0}^{\theta} c_{\mathrm{v}}(r) \mathrm{d} r
$$

Thus, $h$ is a primitive function of $c_{\mathrm{v}}$, normalized in such a way that $h(0)=0$. Since $c_{\mathrm{v}}$ is strictly positive $(c f$. assumption (3.8a) later on), $h$ is strictly increasing. Thus, we are entitled to define

$$
\Theta(w):=\left\{\begin{array}{ll}
h^{-1}(w) \text { if } w \geq 0, \\
0 & \text { if } w<0,
\end{array} \quad \mathcal{K}(e, w):=\frac{\mathbb{K}(e, \Theta(w))}{c_{\mathrm{v}}(\Theta(w))},\right.
$$


where $h^{-1}$ here denotes the inverse function to $h$. With transformations (3.4) and (3.5), the classical formulation (2.2) of the SBV-brittle delamination system (with $\sigma$ from (2.8) and the additional SBV-gradient regularization in $(2.10))$, turns into

$$
\begin{array}{ll}
-\operatorname{div}\left(\mathrm{DR}_{2}(e(\dot{u}))+\mathrm{DW}_{2}(e(u))-\mathbb{B} \Theta(w)+\mathrm{DW}_{p}(e(u))\right)=F & \text { in }(0, T) \times(\Omega \backslash \Gamma), \\
\dot{w}-\operatorname{div}(\mathcal{K}(e(u), w) \nabla w)=e(\dot{u}): \mathbb{D}: e(\dot{u})-\Theta(w) \mathbb{B}: e(\dot{u})+H & \text { in }(0, T) \times(\Omega \backslash \Gamma), \\
u=0 & \text { on }(0, T) \times \Gamma_{\mathrm{D}}, \\
\left.\sigma(u, \dot{u}, w)\right|_{\Gamma_{\mathrm{N}}} \mathrm{n}=f & \text { on }(0, T) \times \Gamma_{\mathrm{N}}, \\
(\mathcal{K}(e(u), w) \nabla w) \mathrm{n}=h & \text { on }(0, T) \times \partial \Omega, \\
\llbracket \sigma(u, \dot{u}, w) \rrbracket \mathrm{n}=0 & \text { on }(0, T) \times \Gamma, \\
\llbracket u \rrbracket \cdot \mathrm{n} \geq 0 & \text { on }(0, T) \times \Gamma, \\
\left.\sigma(u, \dot{u}, w)\right|_{\Gamma} \mathrm{n} \cdot \mathrm{n} \geq 0 \quad \text { wherever } z(\cdot)=0 & \text { on }(0, T) \times \Gamma, \\
\left.\sigma(u, \dot{u}, w)\right|_{\Gamma} \mathrm{n} \cdot \llbracket u \rrbracket=0 & \text { on }(0, T) \times \Gamma, \\
\partial I_{(-\infty, 0]}(\dot{z})+\partial_{z} \Phi(u, z)+\partial \mathcal{G}_{\mathrm{b}}(z)-a_{0}-a_{1} \ni 0 & \text { on }(0, T) \times \Gamma, \\
\frac{1}{2}\left(\left.\mathcal{K}(e(u), w) \nabla w\right|_{\Gamma} ^{+}+\left.\mathcal{K}(e(u), w) \nabla w\right|_{\Gamma} ^{-}\right) \cdot \mathrm{n}+\eta(\llbracket u \rrbracket, z) \llbracket \Theta(w) \rrbracket=0 & \text { on }(0, T) \times \Gamma, \\
\llbracket \mathcal{K}(e(u), w) \nabla w \rrbracket \cdot \mathrm{n}=-a_{1} \dot{z} & \text { on }(0, T) \times \Gamma,
\end{array}
$$

where $\mathrm{W}_{2}(e):=\frac{1}{2} e: \mathbb{C}: e$ and $\mathrm{W}_{p}(e):=\frac{1}{p}|e|^{p-2} e: \mathbb{H}: e$ with $p>d$ in (3.6a), and we have introduced the placeholder

$$
\mathbb{B}:=\mathbb{C}: \mathbb{E}
$$

Furthermore, in the momentum equation and in the enthalpy equation, we have incorporated the notation from (1.1) for the dissipation potentials. With slight abuse, we also write

$$
\sigma(u, v, w):=\sigma(u, v, \Theta(w))=\left[\mathrm{DR}_{2}(e(v))+\mathrm{DW}_{2}(e(u))-\mathbb{B} \Theta(w)+\mathrm{DW}_{p}(e(u))\right] .
$$

With obvious changes, one also obtains the classical enthalpy reformulation of the SBV-adhesive (cf. (2.11) and (2.12)), and of the Modica-Mortola adhesive ( $c f .(2.14)$ ) contact systems.

\subsection{Assumptions on the domain and the given data}

Assumptions on the reference domain $\Omega$. We suppose that

- $\Omega \subset \mathbb{R}^{d}, d \geq 2$, is bounded, $\Omega_{-}, \Omega_{+}, \Omega$ are Lipschitz domains, $\Omega_{+} \cap \Omega_{-}=\emptyset$,

- $\partial \Omega=\Gamma_{\mathrm{D}} \cup \Gamma_{\mathrm{N}}, \Gamma_{\mathrm{D}}, \Gamma_{\mathrm{N}}$ open subsets in $\partial \Omega, \Gamma_{\mathrm{D}} \cap \Gamma_{\mathrm{N}}=\emptyset, \mathcal{H}^{d-1}\left(\Gamma_{\mathrm{D}}\right)>0$,

- $\Gamma \subset \mathbb{R}^{d-1}$ is a convex domain, contained in a hyperplane of $\mathbb{R}^{d}$, such that in particular $\mathcal{H}^{d-1}(\Gamma)=\mathcal{L}^{d-1}(\Gamma)>0$,

where $\mathcal{H}^{d-1}$ and $\mathcal{L}^{d-1}$ respectively denote the $(d-1)$-dimensional Hausdorff and Lebesgue measures.

Assumptions on the given data. We impose the following conditions on $c_{\mathrm{v}}, \mathbb{K}$, and $\eta$ :

$c_{\mathrm{v}}:[0,+\infty) \rightarrow \mathbb{R}^{+}$continuous,

$\exists \omega_{1} \geq \omega>\frac{2 d}{d+2}, c_{1} \geq c_{0}>0$ such that $\forall \theta \in \mathbb{R}^{+}: \quad c_{0}(1+\theta)^{\omega-1} \leq c_{\mathrm{v}}(\theta) \leq c_{1}(1+\theta)^{\omega_{1}-1}$,

$\mathbb{K}: \mathbb{R}^{d \times d} \times \mathbb{R} \rightarrow \mathbb{R}^{d \times d}$ is bounded, continuous, and

$$
\inf _{(e, w, \xi) \in \mathbb{R}_{\mathrm{sym}}^{d \times d} \times \mathbb{R} \times \mathbb{R}^{d},|\xi|=1} \mathcal{K}(e, w) \xi: \xi>0,
$$


and we require that

$$
\begin{aligned}
& \eta: \Gamma \times\left(\mathbb{R}^{d} \times \mathbb{R}\right) \rightarrow \mathbb{R}^{+} \text {is a Carathéodory function such that } \\
& \exists C_{\eta}>0 \exists \sigma_{1}, \sigma_{2}>0 \text { such that } \forall(x, v, z) \in \Gamma \times \mathbb{R}^{d} \times \mathbb{R}: \quad|\eta(x, v, z)| \leq C_{\eta}\left(1+|v|^{\sigma_{1}}+|z|^{\sigma_{2}}\right) .
\end{aligned}
$$

In particular, notice that any polynomial growth of $\eta$ w.r.t. the variables $(v, z)$ is admissible.

Remark 3.2. It is immediate to deduce from (3.8b) that

$$
\exists C_{\theta}^{1}, C_{\theta}^{2}>0 \quad \forall w \in \mathbb{R}^{+}: \quad\left(C_{\theta}^{1} w+1\right)^{1 / \omega_{1}}-1 \leq \Theta(w) \leq\left(C_{\theta}^{2} w+1\right)^{1 / \omega}-1 .
$$

In particular, since $\omega>1$, the right-hand side estimate yields

$$
\Theta(w) \leq C_{\theta}^{2} w .
$$

Moreover, it follows from (3.8b) and (3.8c) and the definition (3.5) of $\mathcal{K}$ that

$$
\exists C_{\mathcal{K}}>0 \quad \forall \xi, \zeta \in \mathbb{R}^{d}: \quad|\mathcal{K}(e, w) \xi: \zeta| \leq C_{\mathcal{K}}|\xi||\zeta| .
$$

Data qualification. We shall suppose for the right-hand sides $F, H, f$, and $h$ that

$$
\begin{aligned}
& F \in L^{2}\left(0, T ; W^{1,2}\left(\Omega \backslash \Gamma ; \mathbb{R}^{d}\right)^{*}\right) \cap W^{1,1}\left(0, T ; W^{1, p}\left(\Omega \backslash \Gamma ; \mathbb{R}^{d}\right)^{*}\right), \\
& f \in L^{2}\left(0, T ; L^{2(d-1) / d}\left(\Gamma_{\mathrm{N}} ; \mathbb{R}^{d}\right)\right) \cap W^{1,1}\left(0, T ; L^{1}\left(\Gamma_{\mathrm{N}} ; \mathbb{R}^{d}\right)\right), \\
& H \in L^{1}\left(0, T ; L^{1}(\Omega)\right), \quad H \geq 0 \text { a.e. in } Q, \\
& h \in L^{1}\left(0, T ; L^{1}(\partial \Omega)\right), \quad h \geq 0 \text { a.e. in }(0, T) \times \partial \Omega .
\end{aligned}
$$

We also introduce the functions (with an abuse of notation, below we write integrals instead of duality pairings)

$\mathrm{F}:(0, T) \rightarrow W^{1, p}\left(\Omega \backslash \Gamma ; \mathbb{R}^{d}\right)^{*},\langle\mathrm{~F}(t), v\rangle:=\int_{\Omega} F(t) \cdot v \mathrm{~d} x+\int_{\Gamma_{\mathrm{N}}} f(t) \cdot v \mathrm{~d} \mathcal{H}^{d-1}$ for $v \in W^{1, p}\left(\Omega \backslash \Gamma ; \mathbb{R}^{d}\right)$,

$\mathrm{H}:(0, T) \rightarrow W^{1, r}\left(\Omega \backslash \Gamma ; \mathbb{R}^{d}\right)^{*},\langle\mathrm{H}(t), v\rangle:=\int_{\Omega} H(t) v \mathrm{~d} x+\int_{\partial \Omega} h(t) v \mathrm{~d} \mathcal{H}^{d-1} \quad$ for $v \in W^{1, r}\left(\Omega \backslash \Gamma ; \mathbb{R}^{d}\right)$,

with $1 \leq r<\frac{d+2}{d+1}, c f .(3.27 \mathrm{c})$ later on. Finally, we impose the following on the initial data

$$
\begin{gathered}
u_{0} \in W_{\Gamma_{\mathrm{D}}}^{1, p}\left(\Omega \backslash \Gamma ; \mathbb{R}^{d}\right), \llbracket u_{0} \rrbracket \cdot \mathrm{n} \geq 0 \text { on }(0, T) \times \Gamma, \\
z_{0} \in L^{\infty}(\Gamma), \quad 0 \leq z_{0} \leq 1 \text { a.e. on } \Gamma, \\
\theta_{0} \in L^{\omega_{1}}(\Omega), \quad \theta_{0} \geq 0 \text { a.e. in } \Omega,
\end{gathered}
$$

where $\omega_{1}$ is the same as in (3.8b). It follows from (3.14c) and (3.8b) that $w_{0}:=h\left(\theta_{0}\right) \in L^{1}(\Omega)$.

\subsection{General energetic formulation}

In the weak formulation for the SBV-brittle delamination system and for its approximations, a crucial role is played by the mechanical part of the overall Helmholtz free energy, i.e. by the functional $\Phi: W^{1, p}\left(\Omega \backslash \Gamma ; \mathbb{R}^{d}\right) \times$ $\mathcal{Z} \rightarrow(-\infty,+\infty]$ (with the space $\mathcal{Z}$ specified below), given by $\Phi(u, z):=\Phi^{\text {bulk }}(u)+\Phi^{\text {surf }}(\llbracket u \rrbracket, z), c f$. (1.4). In fact, the functional $\Phi^{\text {surf }}: L^{2}(\Gamma) \times \mathcal{Z} \rightarrow(-\infty,+\infty]$ is the only contribution in the mechanical energy $\Phi$ to change when passing from the Modica-Mortola, to the SBV-adhesive contact, and to the SBV-brittle delamination systems, whereas the bulk contribution $\Phi^{\text {bulk }}: W^{1, p}\left(\Omega \backslash \Gamma ; \mathbb{R}^{d}\right) \rightarrow[0,+\infty)$ for all of the three models is given by

$$
\Phi^{\text {bulk }}(u):=\int_{\Omega \backslash \Gamma}\left(\mathrm{W}_{2}(e(u))+\mathrm{W}_{p}(e(u))\right) \mathrm{d} x \quad \text { with } \quad \mathrm{W}_{2}(e):=\frac{1}{2} e: \mathbb{C}: e, \mathrm{~W}_{p}(e):=\frac{1}{p}|e|^{p-2} e: \mathbb{H}: e .
$$


In order to specify the surface mechanical energies, we observe that the impenetrability constraint $\llbracket u \rrbracket \cdot \mathrm{n} \geq 0$ on $(0, T) \times \Gamma$ can be reformulated as

$$
\llbracket u(t, x) \rrbracket \in \mathrm{C}(x) \quad \text { for a.a. }(t, x) \in(0, T) \times \Gamma,
$$

upon introducing the multivalued mapping

$$
\mathrm{C}: \Gamma \rightrightarrows \mathbb{R}^{d} \text { s.t. } \mathrm{C}(x)=\left\{v \in \mathbb{R}^{d} ; v \cdot \mathrm{n}(x) \geq 0\right\} \text { for a.a. } x \in \Gamma \text {. }
$$

We will denote by $I_{\mathrm{C}(x)}$ the indicator functional of the closed cone $\mathrm{C}(x)$, and by $\partial I_{\mathrm{C}(x)}$ its (convex analysis) subdifferential. For the definition and basic properties of subdifferentials, the reader may refer, e.g., to [38].

Then, the surface contributions to the mechanical energy are

- for the Modica-Mortola adhesive system:

$$
\begin{aligned}
\Phi^{\text {surf }}=\Phi_{k, m}^{\text {surf }}(\llbracket u \rrbracket, z):= & \int_{\Gamma}\left(I_{\mathrm{C}(x)}(\llbracket u \rrbracket)+J_{k}(\llbracket u \rrbracket, z)+I_{[0,1]}(z)-a_{0} z\right) \mathrm{d} \mathcal{H}^{d-1}+\mathcal{G}_{m}(z) \\
& \text { with } J_{k}(\llbracket u \rrbracket, z):=\frac{k}{2} z|\llbracket u \rrbracket|^{2} \text { and } \mathcal{G}_{m} \text { from }(2.13) .
\end{aligned}
$$

We denote by $\Phi_{k, m}$ the corresponding mechanical energy, defined on $W^{1, p}\left(\Omega \backslash \Gamma ; \mathbb{R}^{d}\right) \times \mathcal{Z}_{\mathrm{MM}}$, with

$$
\mathcal{Z}_{\mathrm{MM}}:=H^{1}(\Gamma)
$$

- for the SBV-adhesive system:

$$
\Phi^{\text {surf }}=\Phi_{k}^{\text {surf }}(\llbracket u \rrbracket, z)=\int_{\Gamma}\left(I_{\mathrm{C}(x)}(\llbracket u \rrbracket)+J_{k}(\llbracket u \rrbracket, z)+I_{[0,1]}(z)-a_{0} z\right) \mathrm{d} \mathcal{H}^{d-1}+\mathcal{G}_{\mathrm{b}}(z)
$$

with

$$
\mathcal{G}_{\mathrm{b}}(z)= \begin{cases}\mathrm{b} \mathcal{H}^{d-2}\left(\mathrm{~J}_{z}\right) & \text { if } z \in \operatorname{SBV}(\Gamma ;\{0,1\}), \\ +\infty & \text { otherwise }\end{cases}
$$

(cf. also (2.9)), where $\mathrm{J}_{z}$ denotes the set of approximate jump points of $z$ ( $c f$. Def. A.15) and, from the calculations for the $\Gamma$-limit passage as $m \rightarrow \infty$ in the Modica-Mortola functionals $\left(\mathcal{G}_{m}\right)_{m}$ (see $[2,50]$ ), it follows that $\mathrm{b}=2 \int_{0}^{1} \xi(1-\xi) \mathrm{d} \xi$. We denote by $\Phi_{k}$ the corresponding mechanical energy, defined on the space $W^{1, p}\left(\Omega \backslash \Gamma ; \mathbb{R}^{d}\right) \times \mathcal{Z}_{\mathrm{SBV}}$, with

$$
\mathcal{Z}_{\mathrm{SBV}}:=\operatorname{SBV}(\Gamma ;\{0,1\})
$$

- for the SBV-brittle system:

$$
\Phi^{\text {surf }}=\Phi_{\mathrm{b}}^{\text {surf }}(\llbracket u \rrbracket, z)=\int_{\Gamma}\left(I_{\mathrm{C}(x)}(\llbracket u \rrbracket)+J_{\infty}(\llbracket u \rrbracket, z)+I_{[0,1]}(z)-a_{0} z\right) \mathrm{d} \mathcal{H}^{d-1}+\mathcal{G}_{\mathrm{b}}(z),
$$

cf. (2.4) for the definition of $J_{\infty}$. We denote by $\Phi_{\mathrm{b}}$ the corresponding mechanical energy, defined on the space $W^{1, p}\left(\Omega \backslash \Gamma ; \mathbb{R}^{d}\right) \times \mathcal{Z}_{\mathrm{SBV}}$.

Exploiting the positive 1-homogeneity of the dissipation potential from (1.1), we now introduce its related dissipation distance, also denoted by $\mathcal{R}_{1}$ from now on, i.e. $\mathcal{R}_{1}: L^{1}(\Gamma) \times L^{1}(\Gamma) \rightarrow[0,+\infty]$ defined (with slight abuse of notation) by

$$
\mathcal{R}_{1}(\tilde{z}-z):=\int_{\Gamma} \mathrm{R}_{1}(\tilde{z}-z) \mathrm{d} \mathcal{H}^{d-1}= \begin{cases}\int_{\Gamma} a_{1}|\tilde{z}-z| \mathrm{d} \mathcal{H}^{d-1} & \text { if } \tilde{z} \leq z \text { a.e. in } \Gamma \\ +\infty & \text { otherwise. }\end{cases}
$$


In view of the bulk term with $p$-growth in (3.15) and the surface energies (3.17) and (3.22), we shall use the following notation for sets of test functions for the weak formulation of the momentum equation

$$
\begin{aligned}
\mathcal{U} & :=\left\{v \in W_{\Gamma_{\mathrm{D}}}^{1, p}\left(\Omega \backslash \Gamma ; \mathbb{R}^{d}\right): \llbracket v(x) \rrbracket \in \mathrm{C}(x) \text { for a.a. } x \in \Gamma\right\} ; \\
\mathcal{U}_{z} & :=\left\{v \in W_{\Gamma_{\mathrm{D}}}^{1, p}\left(\Omega \backslash \Gamma ; \mathbb{R}^{d}\right): \llbracket v(x) \rrbracket \in \mathrm{C}(x), z(x) \llbracket v(x) \rrbracket=0 \text { for a.a. } x \in \Gamma\right\}
\end{aligned}
$$

with a given $z \in L^{1}(\Gamma)$. The former set is used in the adhesive and the latter in the brittle setting.

The enthalpy equation will be formulated as a variational inequality, restricted to positive test functions in order to deal with the quadratic dissipation term on the right-hand side by lower semicontinuity (see also Rem. 3.12). In particular, we shall use test functions in the space

$$
\mathcal{W}:=\mathrm{C}^{0}\left([0, T] ; W^{1, r^{\prime}}(\Omega \backslash \Gamma)\right) \cap W^{1, r^{\prime}}\left(0, T ; L^{r^{\prime}}(\Omega)\right) \subset \mathrm{C}^{0}\left([0, T] ; L^{\infty}(\Gamma)\right)
$$

where $r^{\prime}=\frac{r}{r-1}$ is the conjugate exponent of $r$ in (3.27c) below. Since $1 \leq r<\frac{d+2}{d+1}$, by trace embedding (3.2) the inclusion in (3.26) holds. In turn, we may mention that the $L^{r}\left(0, T ; W^{1, r}(\Omega \backslash \Gamma)\right)$-regularity for $w$ derives from Boccardo-Gallouët-type estimates [7] on the enthalpy equation, combined with the Gagliardo-Nirenberg inequality. We refer to the proof of the forthcoming Proposition 3.14, and to [57] for all details.

We are now in the position to introduce a general weak solvability notion for a thermal delamination system, i.e. the Modica-Mortola/SBV-adhesive, and SBV-brittle systems, consisting of the weak formulation of the momentum equation, of a mechanical energy inequality, a semistability condition, and of the variational formulation of the enthalpy equation. While the last three items have the same form for each of the delamination systems we consider, we will not give a unified variational formulation of the momentum equation, for it substantially changes when switching from adhesive to brittle delamination (see Lem. 3.11 later on). In particular, let us highlight that in the brittle case the set of test functions $\mathcal{U}_{z}$ for the weak formulation of the momentum equation does depend on the $z$-component of the solution.

Definition 3.3 (Energetic solution).

Given a triple of initial data $\left(u_{0}, z_{0}, \theta_{0}\right)$ satisfying (3.14), we call a triple $(u, z, w)$ an energetic solution of a thermal delamination system, if

$$
\begin{aligned}
& u \in L^{\infty}\left(0, T ; W_{\Gamma_{\mathrm{D}}}^{1, p}\left(\Omega \backslash \Gamma ; \mathbb{R}^{d}\right)\right) \cap W^{1,2}\left(0, T ; W_{\Gamma_{\mathrm{D}}}^{1,2}\left(\Omega \backslash \Gamma ; \mathbb{R}^{d}\right)\right), \\
& z \in L^{\infty}((0, T) \times \Gamma) \cap \operatorname{BV}\left([0, T] ; L^{1}(\Gamma)\right), \quad z(t, x) \in[0,1] \text { for a.a. }(t, x) \in(0, T) \times \Gamma, \\
& w \in L^{r}\left(0, T ; W^{1, r}(\Omega \backslash \Gamma)\right) \cap L^{\infty}\left(0, T ; L^{1}(\Omega)\right) \cap \operatorname{BV}\left([0, T] ; W^{1, r^{\prime}}(\Omega \backslash \Gamma)^{*}\right)
\end{aligned}
$$

for every $1 \leq r<\frac{d+2}{d+1}$, the triple $(u, z, w)$ complies with the initial conditions

$$
u(0)=u_{0} \quad \text { a.e. in } \Omega, \quad z(0)=z_{0} \quad \text { a.e. in } \Gamma, \quad w(0)=w_{0} \quad \text { a.e. in } \Omega,
$$

and with

1. the weak formulation of the momentum equation

\section{-in the adhesive case:}

$$
\begin{aligned}
& u(t) \in \mathcal{U} \text { for a.a. } t \in(0, T), \quad \text { and for all } v \in \mathcal{U} \\
& \int_{\Omega \backslash \Gamma}\left(\mathrm{DR}_{2}\left(e(\dot{u}(t))+\mathrm{DW}_{2}(e(u(t)))-\mathbb{B} \Theta(w(t))+\mathrm{DW}_{p}(e(u(t)))\right): e(v-u(t)) \mathrm{d} x\right. \\
& \quad+\int_{\Gamma} k z(t) \llbracket u(t) \rrbracket \cdot \llbracket v-u(t) \rrbracket \mathrm{d} \mathcal{H}^{d-1} \geq\langle\mathrm{F}(t), v-u(t)\rangle \quad \text { for a.a. } t \in(0, T) ;
\end{aligned}
$$


-in the brittle case:

$$
\begin{gathered}
u(t) \in \mathcal{U}_{z(t)} \text { for a.a. } t \in(0, T), \quad \text { and for all } v \in \mathcal{U}_{z(t)} \\
\int_{\Omega \backslash \Gamma}\left(\mathrm{DR}_{2}\left(e(\dot{u}(t))+\mathrm{DW}_{2}(e(u(t)))-\mathbb{B} \Theta(w(t))+\mathrm{DW}_{p}(e(u(t)))\right): e(v-u(t)) \mathrm{d} x\right. \\
\geq\langle\mathrm{F}(t), v-u(t)\rangle \quad \text { for a.a. } t \in(0, T) ;
\end{gathered}
$$

2. semistability for all $t \in(0, T]$

$$
\forall \tilde{z} \in \mathcal{Z}: \quad \Phi(u(t), z(t)) \leq \Phi(u(t), \tilde{z})+\mathcal{R}_{1}(\tilde{z}-z(t)) ;
$$

3. mechanical energy inequality

$$
\begin{aligned}
& \Phi(u(t), z(t))+\int_{0}^{t} 2 \mathcal{R}_{2}(e(\dot{u})) \mathrm{d} s+\operatorname{Var}_{\mathcal{R}_{1}}(z ;[0, t]) \\
& \quad \leq \Phi\left(u_{0}, z_{0}\right)+\int_{0}^{t} \int_{\Omega \backslash \Gamma} \Theta(w) \mathbb{B}: e(\dot{u}) \mathrm{d} x \mathrm{~d} s+\int_{0}^{t}\langle\mathrm{~F}, \dot{u}\rangle \mathrm{d} s \quad \text { for all } t \in[0, T],
\end{aligned}
$$

where we use the notation

$$
\operatorname{Var}_{\mathcal{R}_{1}}\left(\tilde{z} ;\left[t_{1}, t_{2}\right]\right):=\sup \sum_{i=1}^{k} \mathcal{R}_{1}\left(\tilde{z}\left(s_{i}\right)-\tilde{z}\left(s_{i-1}\right)\right) \text { for } \tilde{z} \in L^{1}(\Gamma),\left[t_{1}, t_{2}\right] \subset[0, T],
$$

with the sup taken over all partitions $t_{1}=s_{0}<\ldots<s_{k}=t_{2}$ of the interval $\left[t_{1}, t_{2}\right]$;

4. weak enthalpy inequality

$$
\begin{aligned}
& \langle w(T), \zeta(T)\rangle+\int_{0}^{T} \int_{\Omega \backslash \Gamma} \mathcal{K}(e(u), w) \nabla w \cdot \nabla \zeta-w \dot{\zeta} \mathrm{d} x \mathrm{~d} t+\int_{0}^{T} \int_{\Gamma} \eta(x, \llbracket u \rrbracket, z) \llbracket \Theta(w) \rrbracket \llbracket \zeta \rrbracket \mathrm{d} \mathcal{H}^{d-1} \mathrm{~d} t \\
& \geq \int_{0}^{T} \int_{\Omega \backslash \Gamma}\left(2 \mathrm{R}_{2}(e(\dot{u}))-\Theta(w) \mathbb{B}: e(\dot{u})\right) \zeta \mathrm{d} x \mathrm{~d} t+\iint_{(0, T) \times \Gamma} \frac{\left.\zeta\right|_{\Gamma} ^{+}+\left.\zeta\right|_{\Gamma} ^{-}}{2} \mathrm{~d} \xi_{\dot{z}}^{\text {surf }}(S, t) \\
& \quad+\int_{0}^{T}\langle\mathrm{H}, \zeta\rangle \mathrm{d} t+\int_{\Omega \backslash \Gamma} w_{0} \zeta(0) \mathrm{d} x \quad \text { for all } \zeta \in \mathcal{W} \text { with } \zeta \geq 0 \text { a.e. },
\end{aligned}
$$

where $w_{0}=h\left(\theta_{0}\right)$ and $\xi_{\dot{z}}^{\text {surf }}$ is a measure (=heat produced by rate-independent dissipation) defined by prescribing its values for every closed set of the type $A:=\left[t_{1}, t_{2}\right] \times C \subset[0, T] \times \bar{\Gamma}$ by

$$
\xi_{\dot{z}}^{\text {surf }}(A):=\int_{C} \mathrm{R}_{1}\left(z\left(t_{1}, x\right)-z\left(t_{2}, x\right)\right) \mathrm{d} \mathcal{H}^{d-1}
$$

Notice that, since $w \in \mathrm{BV}\left([0, T] ; W^{1, r^{\prime}}(\Omega \backslash \Gamma)^{*}\right)$, for all $t \in[0, T]$ one has $w(t) \in W^{1, r^{\prime}}(\Omega \backslash \Gamma)^{*}$, so that the first duality pairing on the left-hand side of (3.33) makes sense pointwise.

Remark 3.4 (Consistency with the energetic solutions in the rate-independent case). Note that, without viscosity in the momentum equation and in the isothermal case (i.e., in the case of a purely rate-independent evolution of delamination, $c f$. [58]), the notion of weak solution of Definition 3.3 coincides with the concept of (global) energetic solution introduced in [47], see also [45]. 
Remark 3.5 (Total energy inequality).

Suppose that (3.33) holds as an equality ( $c f$. Thm. 3.10 below). Then, adding the mechanical energy inequality (3.31) (for $t=T$ ), and the weak formulation (3.33) of the enthalpy equation tested by 1 yields a further energy inequality

$$
\Phi(u(T), z(T))+\int_{0}^{T} 2 \mathcal{R}_{2}(e(\dot{u})) \mathrm{d} s+\langle w(T), 1\rangle \leq \Phi\left(u_{0}, z_{0}\right)+\int_{\Omega \backslash \Gamma} w_{0} \mathrm{~d} x+\int_{0}^{T}\langle\mathrm{~F}, \dot{u}\rangle \mathrm{d} t+\int_{0}^{T}\langle\mathrm{H}, 1\rangle \mathrm{d} t,
$$

which involves the enthalpy contribution $\langle w(T), 1\rangle$ as well.

Remark 3.6 (The weak enthalpy inequality).

Variational inequalities akin to (3.33) (and in particular, featuring positive test functions) arise quite naturally in the weak formulation of heat-type equations with quadratic nonlinearities on the right-hand side: we quote for example $[21,22]$ on systems for phase change and nematic liquid crystals, respectively, as well as [20] on a model for compressible, viscous, heat conducting fluids.

Indeed, it is not difficult to verify that, if $z \in \mathrm{BV}\left(0, T ; L^{1}(\Gamma)\right)$ is such that $\dot{z}(t, x)$ exists for almost all $(t, x) \in(0, T) \times \Gamma$, any sufficiently regular function $w$ which fulfills (3.33), is also a supersolution of the boundaryvalue problem $(3.6 \mathrm{~b}),(3.6 \mathrm{e}),(3.6 \mathrm{k}),(3.6 \mathrm{l})$.

Now, we specialize Definition 3.3 to the delamination systems considered in what follows.

Definition 3.7 (Energetic solution of the Modica-Mortola adhesive contact system).

Given a quadruple of initial data $\left(u_{0}, \dot{u}_{0}, z_{0}, \theta_{0}\right)$ satisfying (3.14), we call a triple $(u, z, w)$ an energetic solution to the Modica-Mortola adhesive contact system, if, in addition to (3.27b), we have

$$
z \in L^{\infty}\left(0, T ; \mathcal{Z}_{\mathrm{MM}}\right)
$$

with $\mathcal{Z}_{\mathrm{MM}}$ from (3.18), the triple $(u, z, w)$ fulfills Definition 3.3, with the weak formulation of the momentum inclusion (3.29a), and $\Phi$ replaced by $\Phi_{k, m}$ from (3.17).

Definition 3.8 (Energetic solution of the SBV-adhesive contact system).

Given a quadruple of initial data $\left(u_{0}, \dot{u}_{0}, z_{0}, \theta_{0}\right)$ satisfying $(3.14)$, we call a triple $(u, z, w)$ an energetic solution to the SBV-adhesive contact system, if, in addition to (3.27b), we have

$$
z \in L^{\infty}\left(0, T ; \mathcal{Z}_{\mathrm{SBV}}\right)
$$

with $\mathcal{Z}_{\mathrm{SBV}}$ from $(3.21)$, the triple $(u, z, w)$ fulfills Definition 3.3, with the weak formulation of the momentum inclusion (3.29a), and $\Phi$ replaced by $\Phi_{k}$ from (3.19).

Definition 3.9 (Energetic solution of the SBV-brittle delamination system).

Given a quadruple of initial data $\left(u_{0}, \dot{u}_{0}, z_{0}, \theta_{0}\right)$ satisfying $(3.14)$, we call a triple $(u, z, w)$ an energetic solution to the (Cauchy problem for the) SBV-brittle contact system, if (3.37) holds, the triple $(u, z, w)$ fulfills Definition 3.3, with the weak formulation of the momentum inclusion (3.29b), and $\Phi$ replaced by $\Phi_{\mathrm{b}}$ from (3.22).

\subsection{The energy and enthalpy equalities}

For the adhesive systems it is possible to prove even equalities in the energy inequalities (3.31), (3.35) and in the enthalpy inequality (3.33), also dropping the positivity restriction on the test functions.

Theorem 3.10 (Energy and enthalpy equalities for the adhesive systems).

Assume (3.7), (3.8), (3.12), and (3.14). Then, the Modica-Mortola adhesive and the SBV-adhesive contact systems admit energetic solutions (in the sense of Defs. 3.7 and 3.8) for which the mechanical energy inequality (3.31) and the total energy inequality (3.35) hold as equalities, and so does the enthalpy inequality (3.33) for any test function in $\mathcal{W}$. 
The proof will be given in Section 4.3 for SBV-adhesive contact, i.e. for the energy $\Phi_{k}$ from (3.19) for any $k>0$ fixed. For Modica-Mortola adhesive contact, i.e. with $\Phi_{k, m}$ from (3.17) for any $m, k>0$ fixed, one uses exactly the same arguments. While the mechanical energy estimate (3.31) is obtained by passing to the limit in an approximate mechanical energy inequality exploiting lower semicontinuity, these arguments amount to first showing the opposite relation in (3.31) by means of a Riemann-sum technique (developed in Sect. 4.3), applied to the momentum balance and the semistability inequality. This yields the mechanical energy equality. Using the latter, we are then able to deduce convergence of the quadratic viscous dissipation term on the right-hand side of (3.33), along a sequence of approximate solutions. This convergence is crucial to obtain the enthalpy equality. Finally, summing the mechanical and enthalpy equalities leads to the total energy equality.

In fact, in order to obtain the opposite relation in the mechanical energy inequality for the adhesive models, we will not employ the momentum balance as a variational inequality but consider its reformulation as a subdifferential inclusion, as stated in the following.

Lemma 3.11 (Subdifferential formulation of the momentum equation). Assume (3.7).

1. For $I_{\mathrm{C}}$ from (3.16) and $J_{k}$ from (3.17) consider the functionals

$$
\begin{aligned}
& \mathcal{I}_{\mathrm{C}}: W^{1, p}\left(\Omega \backslash \Gamma ; \mathbb{R}^{d}\right) \rightarrow[0,+\infty], \quad \mathcal{I}_{\mathrm{C}}(u)=\int_{\Gamma} I_{\mathrm{C}(x)}(\llbracket u(x) \rrbracket) \mathrm{d} \mathcal{H}^{d-1}, \\
& \mathcal{J}_{k}: W^{1, p}\left(\Omega \backslash \Gamma ; \mathbb{R}^{d}\right) \times L^{\infty}(\Gamma) \rightarrow[0,+\infty], \quad \mathcal{J}_{k}(u, z)=\int_{\Gamma} J_{k}(\llbracket u \rrbracket, z) \mathrm{d} \mathcal{H}^{d-1}=\frac{k}{2} \int_{\Gamma} z|\llbracket u \rrbracket|^{2} \mathrm{~d} \mathcal{H}^{d-1}, \\
& \mathcal{F}_{k}(u, z):=\mathcal{I}_{\mathrm{C}}(u)+\mathcal{J}_{k}(u, z),
\end{aligned}
$$

with subdifferentials $\partial \mathcal{I}_{\mathrm{C}}, \partial_{u} \mathcal{J}_{k}, \partial_{u} \mathcal{F}_{k}: W^{1, p}\left(\Omega \backslash \Gamma ; \mathbb{R}^{d}\right) \rightrightarrows W^{1, p}\left(\Omega \backslash \Gamma ; \mathbb{R}^{d}\right)^{*}\left(\partial_{u}\right.$ denoting the subdifferential w.r.t. u). Then, the sum rule

$$
\begin{gathered}
\partial_{u} \mathcal{F}_{k}(u, z)=\partial \mathcal{I}_{\mathrm{C}}(u)+\partial_{u} \mathcal{J}_{k}(u, z) \quad \text { holds for all }(u, z) \in W^{1, p}\left(\Omega \backslash \Gamma ; \mathbb{R}^{d}\right) \times L^{\infty}(\Gamma), \quad \text { i.e. } \\
\lambda \in \partial_{u} \mathcal{F}_{k}(u, z) \Leftrightarrow \exists \ell \in \partial \mathcal{I}_{\mathrm{C}}(u) \text { s.t. } \forall v \in W^{1, p}\left(\Omega \backslash \Gamma ; \mathbb{R}^{d}\right) \quad\langle\lambda, v\rangle=\langle\ell, v\rangle+\int_{\Gamma} k z \llbracket u \rrbracket \cdot \llbracket v \rrbracket \mathrm{d} \mathcal{H}^{d-1},
\end{gathered}
$$

and (3.29a) is equivalent to

$$
\begin{aligned}
& \text { for all } v \in W^{1, p}\left(\Omega \backslash \Gamma ; \mathbb{R}^{d}\right) \text {, for a.a. } t \in(0, T) \text { : } \\
& \int_{\Omega \backslash \Gamma}\left(\mathrm{DR}_{2}(e(\dot{u}(t)))+\mathrm{DW}_{2}(e(u(t)))-\mathbb{B} \Theta(w(t))+\mathrm{DW}_{p}(e(u(t)))\right): e(v) \mathrm{d} x
\end{aligned}
$$

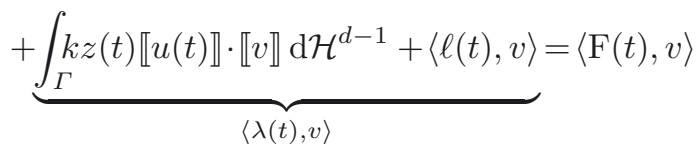

with $\ell \in L^{p^{\prime}}\left(0, T ; W^{1, p}\left(\Omega \backslash \Gamma ; \mathbb{R}^{d}\right)^{*}\right)$ such that $\ell(t) \in \partial \mathcal{I}_{\mathrm{C}}(u(t))$ for a.a.t $\in(0, T)$

and $\lambda \in L^{p^{\prime}}\left(0, T ; W^{1, p}\left(\Omega \backslash \Gamma ; \mathbb{R}^{d}\right)^{*}\right)$ such that $\lambda(t) \in \partial_{u} \mathcal{F}_{k}(u(t), z(t))$ for a.a.t $\in(0, T)$,

where $p^{\prime}=\frac{p}{p-1}$ is the conjugate exponent of $p$.

2. For $I_{\mathrm{C}}$ from (3.16) and $J_{\infty}$ from (2.4) consider the functionals

$$
\begin{aligned}
& \mathcal{J}_{\infty}: W^{1, p}\left(\Omega \backslash \Gamma ; \mathbb{R}^{d}\right) \times L^{\infty}(\Gamma) \rightarrow[0,+\infty], \quad \mathcal{J}_{\infty}(u, z):=\int_{\Gamma} J_{\infty}(\llbracket u(x) \rrbracket, z(x)) \mathrm{d} \mathcal{H}^{d-1}, \\
& \mathcal{F}_{\infty}(u, z):=\mathcal{I}_{\mathrm{C}}(u)+\mathcal{J}_{\infty}(u, z) .
\end{aligned}
$$


Then, $(3.29 \mathrm{~b})$ can be reformulated as

for all $v \in W^{1, p}\left(\Omega \backslash \Gamma ; \mathbb{R}^{d}\right)$ and a.a. $t \in(0, T)$ :

$$
\begin{aligned}
& \int_{\Omega \backslash \Gamma}\left(\mathrm{DR}_{2}\left(e(\dot{u}(t))+\mathrm{DW}_{2}(e(u(t)))-\mathbb{B} \Theta(w(t))+\mathrm{DW}_{p}(e(u(t)))\right): e(v) \mathrm{d} x+\langle\lambda(t), v\rangle=\langle\mathrm{F}(t), v\rangle\right. \\
& \text { with } \lambda \in L^{p^{\prime}}\left(0, T ; W^{1, p}\left(\Omega \backslash \Gamma ; \mathbb{R}^{d}\right)^{*}\right) \text { such that } \lambda(t) \in \partial_{u} \mathcal{F}_{\infty}(u(t), z(t)) \text { for a.a.t } \in(0, T) .
\end{aligned}
$$

Observe that the sum rule (3.41) holds thanks to the Rockafellar-Moreau theorem, see e.g. ([38], p. 200, Thm. 1), since $\mathcal{J}_{k}(\cdot, z)$ is smooth. For $\mathcal{F}_{\infty}$ we only have $\partial \mathcal{I}_{\mathrm{C}}+\partial_{u} \mathcal{J}_{\infty} \subset \partial_{u} \mathcal{F}_{\infty}$, whereas the converse inclusion in fact may not hold.

The analog of Theorem 3.10 cannot be obtained for the SBV-brittle delamination system, where already the strategy to gain the mechanical energy balance fails, and hence the enthalpy equality seems to be out of reach. The reasons for this are expounded in Remark 4.9 below, where we also discuss a possible integration of the weak formulation (3.33) of the enthalpy equation, by means of the concept of defect measures.

Remark 3.12 (Defect measure formulation of the enthalpy equation in the brittle case).

In our approach, the failure of equality in the weak formulation (3.33) of the enthalpy equation is due to a lack of strong compactness in $L^{2}\left(0, T ; L^{2}\left(\Omega ; \mathbb{R}^{d \times d}\right)\right)$ for $\left(e\left(\dot{u}_{k}\right)\right)_{k}$, where $\left(u_{k}, z_{k}, w_{k}\right)_{k}$ is a sequence of solutions to the SBV-adhesive contact problems with which we approximate as $k \rightarrow \infty$ the SBV-brittle delamination system. Therefore, the passage to the limit as $k \rightarrow \infty$ in the quadratic viscous dissipation term on the righthand side of the enthalpy equalities (by Thm. 3.10) for the SBV-adhesive contact systems, solely relies on lower semicontinuity arguments, $c f$. the proof of Theorem 5.2.

Nonetheless, one can consider the limit in the sense of measures of the sequence $\left(2 \mathrm{R}_{2}\left(e\left(\dot{u}_{k}\right)\right)\right)_{k}$ : it is a Radon measure $\mu_{0}$ on $[0, T] \times \bar{\Omega}$. Taking the limit of (3.33) as $k \rightarrow \infty$ then leads to

$$
\begin{aligned}
& \langle w(T), \zeta(T)\rangle+\int_{0}^{T} \int_{\Omega} \mathcal{K}(e(u), w) \nabla w \cdot \nabla \zeta-w \dot{\zeta} \mathrm{d} x \mathrm{~d} t+\int_{0}^{T} \int_{\Gamma} \eta(x, \llbracket u \rrbracket, z) \llbracket \Theta(w) \rrbracket \llbracket \zeta \rrbracket \mathrm{d} \mathcal{H}^{d-1} \mathrm{~d} t \\
& =\int_{0}^{T} \int_{\Omega}\left(2 \mathrm{R}_{2}(e(\dot{u}))-\Theta(w) \mathbb{B}: e(\dot{u})\right) \zeta \mathrm{d} x \mathrm{~d} t+\iint_{(0, T) \times \Gamma} \frac{\left.\zeta\right|_{\Gamma} ^{+}+\left.\zeta\right|_{\Gamma} ^{-}}{2} \mathrm{~d} \xi_{\dot{z}}^{\text {surf }}(S, t) \\
& \quad+\iint_{(0, T) \times \Omega} \zeta \mathrm{d} \mu+\int_{0}^{T}\langle\mathrm{H}, \zeta\rangle \mathrm{d} t+\int_{\Omega \backslash \Gamma} w_{0} \zeta(0) \mathrm{d} x \quad \text { for all } \zeta \in \mathcal{W},
\end{aligned}
$$

where the measure $\mu$ is given by

$$
\mu=\mu_{0}-2 \mathrm{R}_{2}(e(\dot{u})) \mathrm{d} \mathcal{L},
$$

with $\mathrm{d} \mathcal{L}$ is the Lebesgue measure on $(0, T) \times \Omega$. Following [19,30,52] we refer to $\mu$ as a defect measure, for it represents the defect between the limiting measure $\mu_{0}$ and the dissipation $2 \mathrm{R}_{2}(e(\dot{u}))$. The defect-measure formulation (3.46) complements (3.33), in that it reflects a possible additional energy dissipation of solutions lacking regularity and exhibiting concentration effects. Hence, in the brittle case we could complete the weak enthalpy inequality by coupling it with (3.46) and (3.47).

\subsection{Strategy of the existence proof and uniform a priori estimates}

Here, we provide the general scheme for proving the existence of solutions to (the Cauchy problems for) the Modica-Mortola, SBV-adhesive, and SBV-brittle delamination systems, upon taking the limit in a suitable approximate problem: i.e., passing to the limit either with a time-discretization scheme to the Modica-Mortola system, or with the Modica-Mortola system to the SBV-adhesive system, or with the SBV-adhesive system to the SBV-brittle system. We will refer to the latter limit passage as the brittle limit, and to the former two passages as the adhesive limit(s). 
Notation 3.13. Hereafter, we shall suppose that the parameters $m$ and $k$ vary in $\mathbb{N}$. This will allow us to directly consider sequences $\left(u_{m}, z_{m}, w_{m}\right)_{m}$ of solutions to the Modica-Mortola delamination system (where we omit the dependence on $k$ for notational simplicity), when taking the limit as $m \rightarrow \infty$, or sequences $\left(u_{k}, z_{k}, w_{k}\right)_{k}$ of solutions to the SBV-adhesive contact system, when taking the limit as $k \rightarrow \infty$.

In performing the aforementioned passages to the limit, we shall always follow these steps:

Step 0. a priori estimates and compactness for the approximate solutions;

Step 1. proof of the weak formulation of the momentum equation. To this aim, we shall rely on the subdifferential reformulations of Lemma 3.11, and in all of the adhesive limits, use techniques from maximal monotone operator theory to identify the weak limits of the nonlinear terms. For the brittle limit, we will need to prove Mosco-convergence as $k \rightarrow \infty$ of the functionals $\left(\mathcal{J}_{k}\right)_{k}$ to the functional $\mathcal{J}_{\infty}$. Combining this information with maximal monotone operator techniques, we will handle the passage to the limit in the term $\frac{k}{2} z|\llbracket u \rrbracket|^{2}$ as $k \rightarrow \infty$; Step 2. proof of the semistability condition (3.30), verifying the mutual recovery sequence condition from [48], in Propositions 4.6 and 5.9;

Step 3. proof of the mechanical energy inequality (3.31) by lower semicontinuity arguments;

Step 4. proof of the weak formulation of the enthalpy inequality.

A priori estimates. We conclude this section by collecting the a priori estimates on approximate solutions, which are valid for all of the successive approximations of the SBV-brittle system we shall tackle: the Modica-Mortola approximation of the SBV-adhesive system, and the SBV-adhesive approximation of the SBVbrittle system. In order to state such estimates in a unified way, we consider a generic sequence $\left(u_{n}, z_{n}, w_{n}\right)_{n}$ of energetic solutions to the thermal delamination system driven by a sequence $\left(\Phi_{n}\right)_{n}$ of energy functionals $\Phi_{n}: W^{1, p}\left(\Omega \backslash \Gamma ; \mathbb{R}^{d}\right) \times \mathcal{Z} \rightarrow(-\infty,+\infty]$. More specifically, when considering

(a1) the Modica-Mortola approximation of the SBV-adhesive system, we have the energies $\left(\Phi_{k, m}\right)_{m}$, and $\mathcal{Z}=\mathcal{Z}_{\mathrm{MM}}$ : we shall consider the energetic solutions $\left(u_{m}, z_{m}, w_{m}\right)_{m}$ (for notational simplicity, we omit their dependence on $k \in \mathbb{N}$ ), obtained by passing to the limit in the time-discretization scheme of Problem 1 in Appendix A.1;

(a2) the SBV-adhesive approximation of the SBV-brittle system, we have the energies $\left(\Phi_{k}\right)_{k}$, and $\mathcal{Z}=\mathcal{Z}_{\mathrm{SBV}}$ : we shall consider the energetic solutions $\left(u_{k}, z_{k}, w_{k}\right)_{k}$ obtained by passing to the limit in the Modica-Mortola approximation, $c f$. Section 4.

We shall call an energetic solution to the Modica-Mortola adhesive (to the adhesive SBV, resp.) delamination system approximable, if it is obtained by passing to the limit in the time-discretization scheme of problem 1 (in the Modica-Mortola approximation, resp.). We can now state the following general result yielding a priori estimates on the family $\left(u_{n}, z_{n}, w_{n}\right)_{n}$.

Proposition 3.14 (A priori estimates).

Assume (3.7), (3.8), (3.12), and let $\left(u_{0}, \theta_{0}, z_{0}\right)$ be a triple of initial data complying with (3.14). Suppose in addition that $\left(u_{0}, z_{0}\right)$ comply with the semistability (3.30) with the energy $\Phi_{n}$, i.e.

$$
\Phi_{n}\left(u_{0}, z_{0}\right) \leq \Phi_{n}\left(u_{0}, \tilde{z}\right)+\mathcal{R}_{1}\left(\tilde{z}-z_{0}\right) \quad \text { for all } \tilde{z} \in \mathcal{Z} \text {. }
$$

Let $\left(u_{n}, z_{n}, w_{n}\right)_{n}$ be a family of (approximable) energetic solutions to the thermal delamination system in the adhesive case (i.e. with (3.29a)), in either of the two cases (a1) and (a2). 
Then, there exist a constant $S>0$ and, for every $1 \leq r<\frac{d+2}{d+1}, S_{r}>0$, such that for all $n \in \mathbb{N}$ the following estimates hold:

$$
\begin{aligned}
& \left\|u_{n}\right\|_{L^{\infty}\left(0, T ; W_{\Gamma_{\mathrm{D}}}^{1, p}\left(\Omega \backslash \Gamma ; \mathbb{R}^{d}\right)\right) \cap W^{1,2}\left(0, T ; W_{\Gamma_{\mathrm{D}}}^{1,2}\left(\Omega \backslash \Gamma ; \mathbb{R}^{d}\right)\right)} \leq S ; \\
& \sup _{t \in[0, T]} \Phi_{n}\left(u_{n}(t), z_{n}(t)\right) \leq S ; \\
& \left\|z_{n}\right\|_{L^{\infty}((0, T) \times \Gamma)} \leq S ; \\
& \left\|z_{n}\right\|_{\mathrm{BV}\left([0, T] ; L^{1}(\Gamma)\right)} \leq S ; \\
& \left\|w_{n}\right\|_{L^{\infty}\left(0, T ; L^{1}(\Omega)\right)} \leq S ; \\
& \left\|w_{n}\right\|_{L^{r}\left(0, T ; W^{1, r}(\Omega \backslash \Gamma)\right)}+\left\|w_{n}\right\|_{\mathrm{BV}\left([0, T] ; W^{1, r^{\prime}}(\Omega \backslash \Gamma)^{*}\right)} \leq S_{r} \quad \text { for any } 1 \leq r<\frac{d+2}{d+1} .
\end{aligned}
$$

We postpone the proof to Appendix A.1.

Remark 3.15 (Extension: more general bulk energies).

The bulk energy densities $\mathrm{W}_{2}(e)=\frac{1}{2} e: \mathbb{C}: e$ and $\mathrm{W}_{p}(e)=\frac{1}{p}|e|^{p-2} e: \mathbb{H}: e$ can be replaced by general strictly convex, Gâteaux-differentiable functions $\mathrm{W}_{n}: \mathbb{R}^{d} \rightarrow \mathbb{R}$ fulfilling suitable growth assumptions from above and below.

\section{Adhesive Contact: from Modica-Mortola to SBV-Regularization}

The main goal of this section is to prove the existence of energetic solutions in the sense of Definition 3.8 for the SBV-adhesive contact model, and precisely the following

Theorem 4.1 (Existence result for SBV-adhesive contact, $k>0$ fixed). Keep $k>0$ fixed. Assume (3.7), (3.8), (3.12), (3.14). Suppose that the initial data $\left(u_{0}, z_{0}\right)$ fulfill

$$
\Phi_{k}\left(u_{0}, z_{0}\right) \leq \Phi_{k}\left(u_{0}, \tilde{z}\right)+\mathcal{R}_{1}\left(\tilde{z}-z_{0}\right) \quad \text { for all } \tilde{z} \in \mathcal{Z}_{\mathrm{SBV}} .
$$

Then, there exists an energetic solution $(u, w, z)$ to the SBV-adhesive contact system, such that $(u, z)$ comply with the semistability (3.30) for all $t \in[0, T]$. Moreover, for this solution the mechanical energy, the enthalpy and the total energy estimates (3.31), (3.33) and (3.35) with $\Phi_{k}$ hold as equalities. Furthermore,

$$
\exists \theta^{*}>0: \inf _{x \in \Omega} \theta_{0}(x) \geq \theta^{*} \Rightarrow \exists \bar{\theta}>0: \inf _{(t, x) \in(0, T) \times \Omega} \theta(t, x)=\inf _{(t, x) \in(0, T) \times \Omega} \Theta(w(t, x)) \geq \bar{\theta} .
$$

To prove this, we apply the following strategy:

1. we start from an existence result for Modica-Mortola adhesive contact, $m, k>0$ fixed (Thm. 4.2),

2. as $m \rightarrow \infty, k>0$ fixed, we show that the energetic solutions of the Modica-Mortola adhesive contact models suitably converge to an energetic solution of the SBV-adhesive contact model (Thm. 4.3),

3. from Proposition 4.7 and Corollary 4.8 ahead, we directly conclude the validity of the mechanical, the enthalpy and the total energy balance as equalities.

Indeed, we have

Theorem 4.2 (Existence for the Modica-Mortola adhesive contact model, $m, k>0$ fixed). Keep $m, k>0$ fixed. Assume (3.7), (3.8), (3.12), (3.14). Suppose that the initial data $\left(u_{0}, z_{0}\right)$ fulfill

$$
\Phi_{k, m}\left(u_{0}, z_{0}\right) \leq \Phi_{k, m}\left(u_{0}, \tilde{z}\right)+\mathcal{R}_{1}\left(\tilde{z}-z_{0}\right) \quad \text { for all } \tilde{z} \in \mathcal{Z}_{\mathrm{MM}} .
$$

Then, there exists an energetic solution $(u, w, z)$ to the Modica-Mortola adhesive contact system, such that $(u, z)$ complies with the semistability (3.30) for all $t \in[0, T]$. Moreover, for such solution the energy estimates (3.31), (3.33) and (3.35) with $\Phi_{k, m}$ hold as equalities. Furthermore, (4.2) holds. 
The proof of Theorem 4.2 follows from passing to the limit in a suitably devised semi-implicit timediscretization scheme, which we present in Appendix A.1. Therein, we will also sketch the main steps of the passage to the limit in the time-discretization, and specifically dwell on the differences between our argument and the arguments in $[54,55]$, where a semi-implicit discretization procedure was also developed for proving existence to adhesive contact models in thermo-visco-elasticity. In particular, we will detail the proof of the semistability condition (3.30), which needs to be carefully handled due to the gradient regularization in the subdifferential inclusion (2.14) for $z$.

Concerning the convergence of the Modica-Mortola approximation to SBV-adhesive contact, we have

Theorem 4.3 (Modica-Mortola approximation of SBV-adhesive contact, $k>0$ fixed).

Keep $k>0$ fixed. Assume (3.7), (3.8), (3.12). Let $\left(u_{m}, w_{m}, z_{m}\right)_{m}$ be a sequence of approximable solutions to the Modica-Mortola adhesive model, supplemented with initial data $\left(u_{m}^{0}, \theta_{m}^{0}, z_{m}^{0}\right)_{m}$ fulfilling (3.14) and (4.3). Suppose that, as $m \rightarrow \infty$

$$
\begin{aligned}
& u_{m}^{0} \rightarrow u_{0} \quad \text { in } W^{1, p}\left(\Omega \backslash \Gamma ; \mathbb{R}^{d}\right), \quad \theta_{m}^{0} \rightarrow \theta_{0} \quad \text { in } L^{\omega_{1}}(\Omega), \quad z_{m}^{0} \stackrel{*}{\rightarrow} z_{0} \quad \text { in } L^{\infty}(\Gamma), \text { and } \\
& \Phi_{k, m}\left(u_{m}^{0}, z_{m}^{0}\right) \rightarrow \Phi_{k}\left(u_{0}, z_{0}\right) .
\end{aligned}
$$

Then, there exist a (not relabeled) subsequence, and a triple $(u, w, z)$, such that the following convergences hold as $m \rightarrow \infty$

$$
\begin{aligned}
& u_{m} \rightarrow u \\
& u_{m} \rightarrow u \\
& z_{m} \stackrel{*}{\rightarrow} z \\
& z_{m}(t) \stackrel{*}{\rightarrow} z(t) \\
& z_{m}(t) \rightarrow z(t) \\
& z_{m} \rightarrow z \\
& w_{m} \rightarrow w \\
& w_{m} \rightarrow w \\
& w_{m}(t) \rightarrow w(t) \\
& \Theta\left(w_{m}\right) \rightarrow \Theta(w) \\
& \text { in } L^{\infty}\left(0, T ; W_{\Gamma_{\mathrm{D}}}^{1, p}\left(\Omega \backslash \Gamma ; \mathbb{R}^{d}\right)\right) \cap W^{1,2}\left(0, T ; W_{\Gamma_{\mathrm{D}}}^{1,2}\left(\Omega \backslash \Gamma ; \mathbb{R}^{d}\right)\right) \text {, } \\
& \text { in } \mathrm{C}^{0}\left([0, T] ; W_{\Gamma_{\mathrm{D}}}^{1-\epsilon, p}\left(\Omega \backslash \Gamma ; \mathbb{R}^{d}\right)\right) \text { for all } \epsilon \in(0,1] \text {, } \\
& \text { in } L^{\infty}(0, T ; \operatorname{SBV}(\Gamma ;\{0,1\})) \cap L^{\infty}((0, T) \times \Gamma) \text {, } \\
& \text { in } \operatorname{SBV}(\Gamma ;\{0,1\}) \cap L^{\infty}(\Gamma) \text {, } \\
& \text { in } L^{q}(\Gamma) \text { for all } 1 \leq q<\infty \text { for all } t \in[0, T] \text {, } \\
& \text { in } L^{q}\left(0, T ; L^{q}(\Gamma)\right) \text { for all } 1 \leq q<\infty \text {, } \\
& \text { in } L^{r}\left(0, T ; W^{1, r}(\Omega \backslash \Gamma)\right) \text {, } \\
& \text { in } L^{r}\left(0, T ; W^{1-\epsilon, r}(\Omega \backslash \Gamma)\right) \cap L^{q}\left(0, T ; L^{1}(\Omega)\right) \text { for all } \epsilon \in(0,1], 1 \leq q<\infty \text {, } \\
& \text { in } W^{1, r^{\prime}}(\Omega \backslash \Gamma)^{*} \text { for all } t \in[0, T] \text {, } \\
& \llbracket \Theta\left(w_{m}\right) \rrbracket \rightarrow \llbracket \Theta(w) \rrbracket \\
& \text { in } L^{2}\left(0, T ; L^{2}(\Omega)\right) \text {, } \\
& \text { in } L^{r \omega}\left(0, T ; L^{(s-\epsilon) \omega}(\Gamma)\right) \text { for all } 0<\epsilon \leq s-1 \text {, }
\end{aligned}
$$

and $(u, w, z)$ is an energetic solution to the SBV-adhesive contact system. Furthermore, (4.2) holds for $\theta=\Theta(w)$.

Before giving the proof, let us recall the well-known $\Gamma$-convergence theorem for the static functionals $\left(\mathcal{G}_{m}\right)_{m}$ proved in $[50,51]$. It will be exploited for the convergence results $(4.6 \mathrm{e}),(4.6 \mathrm{f})$.

Theorem $4.4([50,51])$. Let $\left(\zeta_{m}\right)_{m} \subset H^{1}(\Gamma)$ fulfill

$$
\sup _{m \in \mathbb{N}} \mathcal{G}_{m}\left(\zeta_{m}\right)<\infty
$$

Then, the sequence $\left(\zeta_{m}\right)_{m}$ is precompact in $L^{1}(\Gamma)$ and every limit point belongs to $\operatorname{SBV}(\Gamma ;\{0,1\})$. Moreover, the functionals $\left(\mathcal{G}_{m}\right)_{m} \Gamma$-converge in $L^{1}(\Gamma)$ as $m \rightarrow \infty$ to the functional $\mathcal{G}_{\mathrm{b}}(3.20)$, i.e.

$\Gamma$-liminf inequality: for all $\zeta \in \operatorname{SBV}(\Gamma ;\{0,1\})$ and $\left(\zeta_{m}\right)_{m} \subset H^{1}(\Gamma)$ with $\zeta_{m} \rightarrow \zeta$ in $L^{1}(\Gamma)$ there holds

$$
\liminf _{m \rightarrow \infty} \mathcal{G}_{m}\left(\zeta_{m}\right) \geq \mathcal{G}_{\mathrm{b}}(\zeta)
$$


$\Gamma$-limsup inequality: for every $\zeta \in \operatorname{SBV}(\Gamma ;\{0,1\})$ there exists $\left(\zeta_{m}\right)_{m} \subset H^{1}(\Gamma)$ with $\zeta_{m} \rightarrow \zeta$ in $L^{1}(\Gamma)$ and $\lim \sup _{m \rightarrow \infty} \mathcal{G}_{m}\left(\zeta_{m}\right) \leq \mathcal{G}_{\mathrm{b}}(\zeta)$.

Theorem 4.4 will also serve as a building block for the limit passage in the semistability condition. Anyhow, let us observe that it will not be sufficient to pass to the limit in the semistability condition. This is ultimately due to the fact that the rate-independent delamination process is non-static. Hence, taking the limit of (3.30) as $m \rightarrow \infty$ requires the construction of a sequence which mutually recovers

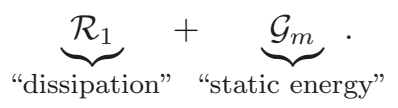

Such a construction of the mutual recovery sequence will be carried out in Section 4.2.

We now develop the proof of Theorem 4.3, following the steps outlined in Section 3.5.

Step 0. Selection of converging subsequences. Estimates (3.48)-(3.53) hold for the sequence $\left(u_{m}, w_{m}\right.$, $\left.z_{m}\right)_{m}$. Convergences (4.6a) and (4.6b) follow from standard weak and strong compactness results $(c f$. the Aubin-Lions type theorems in [59], Cors. 4 and 5). Taking into account that $p>d \geq 2$, Sobolev trace theorems (cf. (3.2)) and embedding results, from (4.6b) we deduce that

$$
\llbracket u_{m} \rrbracket \rightarrow \llbracket u \rrbracket \quad \text { in } \mathrm{C}^{0}\left([0, T] ; \mathrm{C}^{0}\left(\Gamma ; \mathbb{R}^{d}\right)\right) .
$$

As for $\left(z_{m}\right)_{m}$, the $L^{\infty}$-convergence in (4.6c) ensues from (3.50) via the Banach-Alaoglu theorem. To obtain the weak ${ }^{*}$-SBV convergences in (4.6c) and (4.6d), we exploit estimate (3.49), which implies that $\mathcal{G}_{m}\left(z_{m}(t)\right) \leq C$ for a constant independent of $m$ and $t$. Therefore, in view of the well-known compactness and $\Gamma$-convergence result for the static Modica-Mortola functional recalled in Theorem 4.4, the sequence $\left(z_{m}(t)\right)_{m}$ is precompact in $L^{1}(\Gamma)$. The strong $L^{q}$-convergence for any $q \in[1, \infty)$, see $(4.6 \mathrm{e})$ and $(4.6 \mathrm{f})$, is then implied by the uniform $L^{\infty}$-bound (3.50). From this we directly conclude

$$
\mathcal{R}_{1}(z(s)-z(t))=\operatorname{Var}_{\mathcal{R}_{1}}(z ;[s, t])=\lim _{m \rightarrow \infty} \operatorname{Var}_{\mathcal{R}_{1}}\left(z_{m} ;[s, t]\right)=\lim _{m \rightarrow \infty} \mathcal{R}_{1}\left(z_{m}(s)-z_{m}(t)\right)
$$

for all $0 \leq s \leq t \leq T$. For the first and the third equality in (4.10) we have used that both $z$ and $z_{m}$ are nonincreasing w.r.t. time. From (4.8) below, we also deduce that $\liminf _{m \rightarrow \infty} \mathcal{G}_{m}\left(z_{m}(t)\right) \geq \mathcal{G}_{\mathrm{b}}(z(t))$ for all $t \in[0, T]$. Then, taking into account (4.6a), (4.6d), (4.6e), and (4.9), we have

$$
\liminf _{m \rightarrow \infty} \Phi_{k, m}\left(u_{m}(t), z_{m}(t)\right) \geq \Phi_{k}(u(t), z(t)) \quad \text { for all } t \in[0, T] .
$$

As for $\left(w_{m}\right)_{m}$, convergences $(4.6 \mathrm{~g})$ and $(4.6 \mathrm{~h})$ are a consequence of estimates (3.52) and (3.53), and of a generalization of the Aubin-Lions theorem to the case of time derivatives as measures (see e.g. [56], Cor. 7.9). Taking into account the a priori bound of $\left(w_{m}(t)\right)_{m}$ in $L^{1}(\Omega)$, we then conclude (4.6i). Furthermore, arguing by interpolation (e.g. via the Gagliardo-Nirenberg inequality), it is possible to derive from (4.6h) that

$$
w_{m} \rightarrow w \quad \text { in } L^{(d+2) / d-\epsilon}\left(0, T ; L^{(d+2) / d-\epsilon}(\Omega)\right) \quad \text { for all } \quad 0<\epsilon \leq \frac{d+2}{d}-1,
$$

see [57], Section 4 for further details. Hence, relying on the growth condition (3.9) for $\Theta$ and on the fact that $\omega>\frac{2 d}{d+2}$, one can tune $\epsilon>0$ in (4.12) in such a way as to obtain (4.6j). Moreover, again taking into account the trace result (3.2), we deduce from (4.6h) that, $\left.\left.w_{m}^{i}\right|_{\Gamma} \rightarrow w^{i}\right|_{\Gamma}$ in $L^{r}\left(0, T ; L^{s-\epsilon}(\Gamma)\right)$ for all $0<\epsilon \leq s-1$ with $s=\frac{(d-1) r}{d-r}$, for $i=+,-$. Therefore, (3.9) ensures (4.6k).

Steps 1 and 2. i.e. the limit passages in the momentum balance and in the semistability condition will be carried out separately in Sections 4.1 and 4.2, respectively. Let us mention in advance that, upon passing to the limit in the momentum balance we shall also prove that $u_{m} \rightarrow u$ strongly in $L^{p}\left(0, T ; W^{1, p}\left(\Omega \backslash \Gamma ; \mathbb{R}^{d}\right)\right)$, cf. Proposition 4.5 later on. 
Step 3. Mechanical energy inequality. We use (4.6a), (4.10), and (4.11) to pass to the limit as $m \rightarrow \infty$ on the left-hand side of the mechanical energy inequality (3.31) for the Modica-Mortola solutions $\left(u_{m}, w_{m}, z_{m}\right)_{m}$. Combining (4.6a) and (4.6j), we have

$$
\Theta\left(w_{m}\right) \mathbb{B}: e\left(\dot{u}_{m}\right) \rightarrow \Theta(w) \mathbb{B}: e(\dot{u}) \quad \text { in } L^{1}\left(0, T ; L^{1}(\Omega)\right) .
$$

This, (4.5), and again (4.6a) enable us to pass to the limit on the right-hand side of (3.31), and thus to conclude that $(u, w, z)$ complies with the mechanical energy inequality for the SBV-adhesive system.

Step 4. Enthalpy inequality. Thanks to convergence (4.6i) we pass to the limit as $m \rightarrow \infty$ in the first term on the left-hand side of (3.33). We deal with the second integral by means of $(4.6 \mathrm{~g})$, which we combine with the convergence $\mathcal{K}\left(e\left(u_{m}\right), w_{m}\right) \rightarrow \mathcal{K}(e(u), w)$ in $L^{q}\left(0, T ; L^{q}(\Omega)\right)$ for all $1 \leq q<\infty$ due to (4.6b), the above mentioned strong convergence $u_{m} \rightarrow u$ in $L^{p}\left(0, T ; W^{1, p}\left(\Omega \backslash \Gamma ; \mathbb{R}^{d}\right)\right)$, (4.6h), and the boundedness of $\mathcal{K}$. To pass to the limit in the surface integral term on the left-hand side, we rely on (4.6k) and on (4.6f) and (4.9), which yield $\eta\left(\llbracket u_{m} \rrbracket, z_{m}\right) \rightarrow \eta(\llbracket u \rrbracket, z)$ in $L^{q}\left(0, T ; L^{q}(\Gamma)\right)$ for all $1 \leq q<\infty$, in view of the at most polynomial growth (3.8e) of $\eta$. The passage to the limit in the first term on the right-hand side of (3.33) is guaranteed by (4.6a) via lower semicontinuity, and by (4.13). For the second term, we observe that

$$
\xi_{\dot{z}_{m}}^{\text {surf }} \rightarrow \xi_{\dot{z}}^{\text {surf }} \quad \text { in the sense of measures on }(0, T) \times \Gamma .
$$

This follows from the fact that $\operatorname{Var}_{\mathcal{R}_{1}}\left(z_{m},[0, T]\right) \rightarrow \operatorname{Var}_{\mathcal{R}_{1}}(z,[0, T])(c f$. (4.10), as well as (4.40) ahead), arguing in the very same way as in [57] proof of Proposition 4.3. Finally, observe that the strong convergence $\theta_{m}^{0} \rightarrow \theta_{0}$ in $L^{\omega_{1}}(\Omega)$ and the growth condition $(3.8 \mathrm{~b})$ yield that $w_{m}^{0}:=h\left(\theta_{m}^{0}\right) \rightarrow w_{0}:=h\left(\theta_{0}\right)$ in $L^{1}(\Omega)$, which allows us to take the limit of the last term on the right-hand side. Thus, the triple $(u, w, z)$ fulfills the enthalpy inequality (3.33).

Positivity of the temperature. Suppose that $\inf _{x \in \Omega} \theta_{0}(x) \geq \theta^{*}>0$ : it follows from convergence (4.4) that there exist $\bar{m} \in \mathbb{N}$ and $\tilde{\theta}>0$ such that $\inf _{x \in \Omega} \theta_{m}^{0}(x) \geq \tilde{\theta}$ for all $m \geq \bar{m}$. Then, by Theorem 4.2 (cf. also (A.30) later on) there exists $\bar{\theta}>0$ with $\inf _{(t, x) \in(0, T) \times \Omega} \theta_{m}(t, x) \geq \bar{\theta}$ for all $m \geq \bar{m}$, and (4.2) ensues from convergence (4.6j). This concludes the proof of Theorem 4.3.

\subsection{Step 1: Limit passage in the momentum balance}

In the following we verify that the weak momentum equation (3.29a) holds for the SBV-adhesive limit system. For this, we aim to take the limit $m \rightarrow \infty$ in (3.29a) for the Modica-Mortola adhesive systems. But as (4.6a) only guarantees weak $W^{1, p}$-convergence of the Modica-Mortola adhesive displacements $\left(u_{m}\right)_{m}$, we cannot directly pass to the limit with the nonlinear term $\int_{\Omega \backslash \Gamma} \mathrm{DW}_{p}\left(e\left(u_{m}(t)\right): e\left(v-u_{m}(t)\right) \mathrm{d} x\right.$. In order to circumvent this difficulty we are going to make use of the equivalent subdifferential inclusions (3.42). For every $m \in \mathbb{N}$ and a.a. $t \in(0, T)$, these involve the elements $\ell_{m}(t) \in \partial_{u} \mathcal{I}_{\mathrm{C}}\left(u_{m}(t)\right)$, $\mathcal{I}_{\mathrm{C}}$ from (3.38), with $\left(u_{m}(t), w_{m}(t), z_{m}(t), \ell_{m}(t)\right)$ fulfilling (3.42) for a.a. $t \in(0, T)$. Here, a comparison of the terms in (3.42) together with estimates (3.48), (3.50), (3.53) yields a uniform bound for the sequence $\left(\ell_{m}\right)_{m} \subset L^{p^{\prime}}\left(0, T ; W^{1, p}\left(\Omega \backslash \Gamma ; \mathbb{R}^{d}\right)^{*}\right)$, i.e. $\sup _{m \in \mathbb{N}}\left\|\ell_{m}\right\|_{L^{p^{\prime}}\left(0, T ; W^{1, p}\left(\Omega \backslash \Gamma ; \mathbb{R}^{d}\right)^{*}\right)} \leq C$, and hence there exists $\ell \in L^{p^{\prime}}\left(0, T ; W^{1, p}\left(\Omega \backslash \Gamma ; \mathbb{R}^{d}\right)^{*}\right)$ such that up to a subsequence

$$
\ell_{m} \rightarrow \ell \quad \text { in } L^{p^{\prime}}\left(0, T ; W^{1, p}\left(\Omega \backslash \Gamma ; \mathbb{R}^{d}\right)^{*}\right) \text { as } m \rightarrow \infty .
$$

Moreover, due to the bound (3.48), there exists $\mu \in L^{p^{\prime}}\left(0, T ; L^{p^{\prime}}(\Omega)\right)$ such that, up to the extraction of a further (not relabeled) subsequence there holds

$$
\mathrm{DW}_{p}\left(e\left(u_{m}\right)\right) \rightarrow \mu \quad \text { in } L^{p^{\prime}}\left(0, T ; L^{p^{\prime}}(\Omega)\right) \text { as } m \rightarrow \infty .
$$

Exploiting convergences (4.6a), (4.6j), (4.15) and (4.16), we obtain that the limit $(u, w, z, \mu, \ell)$ fulfills

$$
\int_{\Omega \backslash \Gamma}\left(\mathrm{DR}_{2}(e(\dot{u}(t)))+\mathrm{DW}_{2}(e(u(t)))-\mathbb{B} \Theta(w(t))+\mu(t)\right): e(v) \mathrm{d} x+\int_{\Gamma} k z(t) \llbracket u(t) \rrbracket \cdot \llbracket v \rrbracket \mathrm{d} \mathcal{H}^{d-1}+\langle\ell(t), v\rangle=\langle\mathrm{F}(t), v\rangle
$$


for all $v \in W^{1, p}\left(\Omega \backslash \Gamma ; \mathbb{R}^{d}\right)$ and a.a. $t \in(0, T)$. Hence, in order to conclude that (4.17) is the momentum inclusion for the SBV-adhesive limit, we have to identify

$$
\mu(t)=\mathrm{DW}_{p}(e(u(t))) \text { and } \quad \ell(t) \in \partial_{u} \mathcal{I}_{\mathrm{C}}(u(t)) \quad \text { for a.a. } t \in(0, T) .
$$

This will follow from a well-known result from maximal monotone operator theory, for the subdifferential

$$
\left.A:=\partial \mathcal{F} \text { with } \mathcal{F}: L^{p}\left(0, T ; W^{1, p}\left(\Omega \backslash \Gamma ; \mathbb{R}^{d}\right)\right) \rightarrow[0,+\infty], \mathcal{F}(v):=\int_{0}^{t} \int_{\Omega \backslash \Gamma} \mathrm{W}_{p}(e(v))\right) \mathrm{d} x+\mathcal{I}_{\mathrm{C}}(v) \mathrm{d} s .
$$

Note, that the identification of the limits in (4.18) will ultimately imply the strong convergence of $\left(u_{m}\right)_{m}$ in $L^{p}\left(0, T ; W^{1, p}\left(\Omega \backslash \Gamma ; \mathbb{R}^{d}\right)\right)$. Hence, we may state the following result:

Proposition 4.5 (Momentum balance for the SBV-adhesive model).

Let (3.7), (3.8), (3.12), and (3.14) hold true. Keep $k \in \mathbb{N}$ fixed. Consider $\left(u_{m}, z_{m}, w_{m}\right)_{m}$ such that $\left(u_{m}, z_{m}, w_{m}\right) \rightarrow(u, z, w)$ as $m \rightarrow \infty$ in the sense of (4.6) and such that, for all $m \in \mathbb{N}$, the triple $\left(u_{m}, z_{m}, w_{m}\right)$ satisfies the Modica-Mortola adhesive momentum inclusion (3.42). Then the limit (u,z,w) fulfills the SBV-adhesive momentum inclusion for a.a. $t \in(0, T)$ and moreover we have $u_{m} \rightarrow u$ even strongly in $L^{p}\left(0, T ; W^{1, p}\left(\Omega \backslash \Gamma ; \mathbb{R}^{d}\right)\right)$.

Proof. To prove (4.18), we apply ([5], p. 356, Lem. 3.57; cf. also Lem. 5.5 ahead) to $A=\partial \mathcal{F}$ from (4.19); in the following we use the placeholder $X=L^{p}\left(0, T ; W^{1, p}\left(\Omega \backslash \Gamma ; \mathbb{R}^{d}\right)\right)$. Consider $u_{m}^{*} \in X^{*}$ defined by the dual pairing $\left\langle u_{m}^{*}, v\right\rangle_{X}:=\int_{0}^{t} \int_{\Omega \backslash \Gamma} \mathrm{DW}_{p}\left(e\left(u_{m}(s)\right)\right): e(v(s)) \mathrm{d} x+\left\langle\ell_{m}(s), v(s)\right\rangle \mathrm{d} s$ for all $v \in X$. It clearly fulfills $u_{m}^{*} \in A\left(u_{m}\right)$ and (4.15) and (4.16) yield that $u_{m}^{*} \rightarrow u^{*}$ in $X^{*}$, with $u^{*}$ defined by $\left\langle u^{*}, v\right\rangle_{X}:=\int_{0}^{t} \int_{\Omega \backslash \Gamma} \mu(s): e(v(s)) \mathrm{d} x+$ $\langle\ell(s), v(s)\rangle \mathrm{d} s$. We now check that $\lim _{\sup _{m \rightarrow \infty}}\left\langle u_{m}^{*}, u_{m}\right\rangle_{X} \leq\left\langle u^{*}, u\right\rangle_{X}$. To do so, we test the reformulation (3.42) of the momentum equation satisfied by $\left(u_{m}, w_{m}, z_{m}\right)$ with $u_{m}$, integrate in time, and take the $\lim \sup _{m \rightarrow \infty}$. Thus,

$$
\begin{aligned}
& \limsup _{m \rightarrow \infty} \int_{0}^{t}\left(\int_{\Omega \backslash \Gamma} \operatorname{DW}_{p}\left(e\left(u_{m}\right)\right): e\left(u_{m}\right) \mathrm{d} x+\left\langle\ell_{m}, u_{m}\right\rangle\right) \mathrm{d} s
\end{aligned}
$$

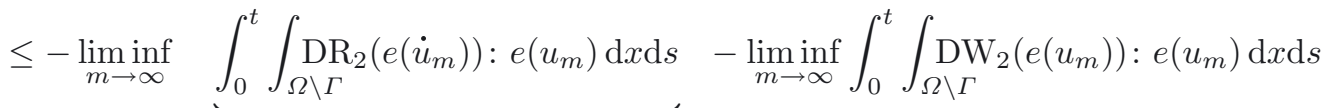

$$
\begin{aligned}
& =\int_{\Omega \backslash \Gamma} \mathrm{R}_{2}\left(e\left(u_{m}(t)\right)\right)-\mathrm{R}_{2}\left(e\left(u_{m}(0)\right)\right) \mathrm{d} x \\
& -\liminf _{m \rightarrow \infty} \int_{0}^{t} \int_{\Gamma} \frac{k}{2} z_{m}\left|\llbracket u_{m} \rrbracket\right|^{2} \mathrm{~d} \mathcal{H}^{d-1} \mathrm{~d} s+\limsup _{m \rightarrow \infty} \int_{0}^{t} \int_{\Omega \backslash \Gamma} \mathbb{B} \Theta\left(w_{m}\right): e\left(u_{m}\right) \mathrm{d} x \mathrm{~d} s+\limsup _{m \rightarrow \infty} \int_{0}^{t}\left\langle\mathrm{~F}, u_{m}\right\rangle \mathrm{d} s \\
& \leq-\int_{0}^{t} \int_{\Omega \backslash \Gamma} \mathrm{DR}_{2}(e(\dot{u})): e(u) \mathrm{d} x \mathrm{~d} s-\int_{0}^{t} \int_{\Omega \backslash \Gamma}\left(\mathrm{DW}_{2}(e(u))-\mathbb{B} \Theta(w)\right): e(u) \mathrm{d} x \mathrm{~d} s \\
& =\int_{\Omega \backslash \Gamma} \mathrm{R}_{2}(e(u(t)))-\mathrm{R}_{2}\left(e\left(u_{0}\right)\right) \mathrm{d} x \\
& -\int_{0}^{t} \int_{\Gamma} \frac{k}{2} z|\llbracket u \rrbracket|^{2} \mathrm{~d} \mathcal{H}^{d-1} \mathrm{~d} s+\int_{0}^{t}\langle\mathrm{~F}, u\rangle \mathrm{d} s \\
& =\int_{0}^{t}\left(\int_{\Omega \backslash \Gamma} \mu: e(u) \mathrm{d} x+\langle\ell, u\rangle\right) \mathrm{d} s .
\end{aligned}
$$

Here, we have used the chain rule formula $\int_{0}^{t} \int_{\Omega \backslash \Gamma} \mathrm{DR}_{2}\left(e\left(\dot{u}_{m}\right)\right): e\left(u_{m}\right) \mathrm{d} x \mathrm{~d} s=\int_{\Omega \backslash \Gamma} \mathrm{R}_{2}\left(e\left(u_{m}(t)\right)\right)-$ $\mathrm{R}_{2}\left(e\left(u_{m}(0)\right)\right) \mathrm{d} x$ for every $m \in \mathbb{N}$, and its analogue in the limit $m \rightarrow \infty$. Then, the second inequality follows 
from convergences (4.6a)-(4.6c) (which in particular yield that $\int_{\Omega \backslash \Gamma} \mathrm{R}_{2}\left(e\left(u_{m}(t)\right)\right) \mathrm{d} x \rightarrow \int_{\Omega \backslash \Gamma} \mathrm{R}_{2}(e(u(t))) \mathrm{d} x$ for all $t \in[0, T]$ ), and (4.6j). The last equality is due to (4.17). Thus, we have $u^{*} \in A(u)$ by ([5], p. 356, Lem. 3.57) and the sum rule (3.41) for $A=\partial \mathcal{F}$ yields that there exists $\tilde{\ell} \in X^{*}$ with $\tilde{\ell}(s) \in \partial \mathcal{I}_{\mathrm{C}}(u(s))$ for a.a. $s \in(0, t)$, such that

$$
\left\langle u^{*}, v\right\rangle_{X}=\int_{0}^{t} \int_{\Omega \backslash \Gamma} \mu(s): e(v(s)) \mathrm{d} x+\langle\ell(s), v(s)\rangle \mathrm{d} s=\int_{0}^{t} \int_{\Omega \backslash \Gamma} \operatorname{DW}_{p}(e(u(s))): e(v(s)) \mathrm{d} x+\langle\tilde{\ell}(s), v(s)\rangle \mathrm{d} s
$$

for all $v \in X$. We conclude that $\ell=\tilde{\ell}$ and $\mu=\operatorname{DW}_{p}(e(u))$. Then, (4.18) holds by the fundamental lemma of the calculus of variations, upon choosing $v(s, x):=\varphi(s) v(x)$ for any $\varphi \in \mathrm{C}_{0}^{\infty}(0, t)$ and any $v \in W^{1, p}\left(\Omega \backslash \Gamma ; \mathbb{R}^{d}\right)$. Thus, inserting this in (4.17), we find that the triple $(u, w, z)$ complies with (3.42), and hence (3.29a) holds true.

\subsection{Step 2: Passage to the limit in the semistability condition}

We now prove that the pair $(u, z)$ complies with the semistability condition (3.30) for any test function $\tilde{z} \in \mathcal{Z}_{\mathrm{SBV}}=\operatorname{SBV}(\Gamma ;\{0,1\})$. To do so, we follow a well-established procedure in the analysis of rate-independent systems: We prove that for all $t \in(0, T]$ there exists a mutual recovery sequence (or MRS, for short) $\left(\tilde{z}_{m}\right)_{m} \subset$ $\mathcal{Z}_{\mathrm{MM}}$ (whose dependence on $t$ is omitted) such that $\tilde{z}_{m} \rightarrow \tilde{z}$ in $L^{1}(\Gamma)$ as $m \rightarrow \infty$, and

$$
\begin{aligned}
& \limsup _{m \rightarrow \infty}\left(\Phi_{k, m}\left(u_{m}(t), \tilde{z}_{m}\right)+\mathcal{R}_{1}\left(\tilde{z}_{m}-z_{m}(t)\right)-\Phi_{k, m}\left(u_{m}(t), z_{m}(t)\right)\right) \\
& \leq \Phi_{k}(u(t), \tilde{z})+\mathcal{R}_{1}(\tilde{z}-z(t))-\Phi_{k}(u(t), z(t)) .
\end{aligned}
$$

Since $\Phi_{k, m}\left(u_{m}(t), \tilde{z}_{m}\right)+\mathcal{R}_{1}\left(\tilde{z}_{m}-z_{m}(t)\right)-\Phi_{k, m}\left(u_{m}(t), z_{m}(t)\right) \geq 0$ for all $m \in \mathbb{N}$ and all $t \in[0, T]$ in view of the semistability (3.30) for the Modica-Mortola solutions $\left(u_{m}, z_{m}\right)$, from (4.22) we will immediately deduce the desired semistability for the limit functions $(u, z)$.

Proposition 4.6 (Mutual recovery sequences for the SBV-adhesive systems).

Keep $k \in \mathbb{N}$ fixed. Let (3.7), (3.8), (3.12), and (3.14) hold true. Let $\Phi_{m, k}$ and $\Phi_{k}$ be given by (3.17) and (3.19). Let $\left(u_{m}\right)_{m}$ satisfy (4.6a) and let $\left(z_{m}\right)_{m} \subset \operatorname{SBV}(\Gamma ;\{0,1\})$ with $z_{m}$ semistable for $\Phi_{m, k}\left(u_{m}, \cdot\right)$ fulfill $z_{m} \stackrel{*}{\rightarrow} z$ in $\operatorname{SBV}(\Gamma ;\{0,1\})$. Then, for every $\tilde{z} \in \mathcal{Z}_{\mathrm{SBV}}$ there is a sequence $\left(\tilde{z}_{m}\right)_{m} \subset \mathcal{Z}_{\mathrm{MM}}$ with $\tilde{z}_{m} \rightarrow \tilde{z}$ in $L^{1}(\Gamma)$ such that (4.22) holds.

Proof. We draw the definition of the MRS $\left(\tilde{z}_{m}\right)_{m}$ from the proof of [62], Lemma 3.5 and, for the reader's convenience, we outline here the main steps in the construction, referring to [62] for all details. We suppose that $\Phi_{k}(u(t), \tilde{z})<\infty$ and $\mathcal{R}_{1}(\tilde{z}-z(t))<\infty$, i.e. that $\tilde{z} \leq z(t)$ a.e., otherwise, the recovery sequence is trivial. For (4.22) to hold, it is also necessary that $\Phi_{k, m}\left(u_{m}(t), \tilde{z}_{m}\right)<\infty$ and $\mathcal{R}_{1}\left(\tilde{z}_{m}-z_{m}(t)\right)<\infty$. Therefore, in [62] the construction from the proof of Theorem 4.4 in $[50,51]$ is suitably adapted to accommodate the latter constraint. In particular, one sets

$$
\tilde{z}_{m}:=\max \left\{0, \min \left\{\left(\hat{z}_{m}-\delta_{m}\right), z_{m}(t)\right\}\right\} \quad \text { with } \delta_{m}:=\left\|\hat{z}_{m}-\tilde{z}+z(t)-z_{m}(t)\right\|_{L^{1}(\Gamma)}^{1 / 2} .
$$

Here, $\left(\hat{z}_{m}\right)_{m}$ is the classical recovery sequence used in $[50,51]$ to prove the $\Gamma$-lim sup condition of Theorem 4.4. In particular, this sequence $\left(\hat{z}_{m}\right)_{m} \subset L^{1}(\Gamma)$ fulfills

$$
\hat{z}_{m} \rightarrow \tilde{z} \text { in } L^{1}(\Gamma), \quad \limsup _{m \rightarrow \infty} \mathcal{G}_{m}\left(\hat{z}_{m}\right) \leq \mathcal{G}_{\mathrm{b}}(\tilde{z}) .
$$

By definition, we have $0 \leq \tilde{z}_{m} \leq z_{m}(t) \leq 1$ a.e. on $\Gamma$. It follows from (4.24) and (4.6e) that $\delta_{m} \rightarrow 0$. Exploiting this, it can be shown that $\tilde{z}_{m} \rightarrow \tilde{z}$ in $L^{1}(\Gamma)$, hence $\mathcal{R}_{1}\left(\tilde{z}_{m}-z_{m}(t)\right) \rightarrow \mathcal{R}_{1}(\tilde{z}-z(t))$. Since $\left(\tilde{z}_{m}\right)_{m}$ is bounded in 
$L^{\infty}(\Gamma)$, we immediately have $\tilde{z}_{m} \rightarrow \tilde{z}$ in $L^{q}(\Gamma)$ for all $1 \leq q<\infty$. Combining this convergence with (4.6e) and (4.9), we then infer

$$
\left\{\begin{array}{l}
\lim _{m \rightarrow \infty} \int_{\Gamma} \frac{k}{2}\left(\tilde{z}_{m}-z_{m}(t)\right)\left|\llbracket u_{m}(t) \rrbracket\right|^{2} \mathrm{~d} \mathcal{H}^{d-1}=\int_{\Gamma} \frac{k}{2}(\tilde{z}-z(t))|\llbracket u(t) \rrbracket|^{2} \mathrm{~d} \mathcal{H}^{d-1} \\
\lim _{m \rightarrow \infty} \int_{\Gamma} a_{0}\left(z_{m}(t)-\tilde{z}_{m}\right) \mathrm{d} S=\int_{\Gamma} a_{0}(z(t)-\tilde{z}) \mathrm{d} S
\end{array}\right.
$$

Repeating the very same calculations as in the proof of [62], Lemma 3.5, one can also show that

$$
\limsup _{m \rightarrow \infty}\left(\mathcal{G}_{m}\left(\tilde{z}_{m}\right)-\mathcal{G}_{m}\left(z_{m}(t)\right)\right) \leq \mathcal{G}_{\mathrm{b}}(\tilde{z})-\mathcal{G}_{\mathrm{b}}(z(t)) .
$$

This concludes the proof of (4.22).

\subsection{Bonus: energy and enthalpy equalities in the adhesive case}

In the following we establish that the mechanical energy (3.31), the enthalpy (3.33) and the total energy (3.35) inequalities hold even as equalities in the adhesive setting, $c f$. Theorem 3.10. For the proof, we will confine ourselves to the SBV-adhesive system. Let us stress that the respective equalities indeed hold for the Modica-Mortola adhesive system and they can be proved along the same lines as in what follows, arguing on the approximating system via time-discretization constructed in Appendix A.1, to which we refer for more details.

We start with proving in Proposition 4.7 the opposite relation in the mechanical energy inequality (3.31), for any solution triple $(u, z, w)$ of the SBV-adhesive system. In $[54,57]$ this was obtained by applying a Riemann-sum argument on the semistability inequality and by testing the momentum balance by the solution $\dot{u}$ of the adhesive system. In our setting, however, the momentum balance cannot be tested by $\dot{u}$, as test functions are required to have $W^{1, p}$-regularity in $\Omega \backslash \Gamma$, cf. (3.24). To avoid testing with $\dot{u}$, we adopt the Riemann-sum technique also for the momentum balance: Let $t \in(0, T]$ be arbitrary but fixed. We choose an equidistant partition of the interval $[0, t]$,

$$
0=t_{0}<t_{1}^{N}<\ldots<t_{N}^{N}=t \quad \text { with } \quad t_{i}^{N}-t_{i-1}^{N}=\tau_{N},
$$

such that the adhesive momentum inclusion (3.42) holds at time $t_{i-1}^{N}$ for all $i=1, \ldots, N$. We test (3.42) at $t_{i-1}^{N}$ by the differences $u_{i}^{N}-u_{i-1}^{N}$

$$
\begin{gathered}
\int_{\Omega \backslash \Gamma}\left(\operatorname{DR}_{2}\left(e\left(\dot{u}_{i-1}^{N}\right)\right)+\mathrm{DW}_{2}\left(e\left(u_{i-1}^{N}\right)\right)-\mathbb{B}_{i-1}^{N}+\mathrm{DW}_{p}\left(e\left(u_{i-1}^{N}\right)\right): e\left(u_{i}^{N}-u_{i-1}^{N}\right) \mathrm{d} x\right. \\
+\left\langle\lambda_{i-1}^{N}, u_{i}^{N}-u_{i-1}^{N}\right\rangle=\left\langle F_{i}^{N}, u_{i}^{N}-u_{i-1}^{N}\right\rangle
\end{gathered}
$$

with $\lambda_{i-1}^{N} \in W^{1, p}\left(\Omega \backslash \Gamma ; \mathbb{R}^{d}\right)^{*}$ s.t. $\lambda_{i-1}^{N} \in \partial \mathcal{F}_{k}\left(u_{i-1}^{N}, z_{i-1}^{N}\right)$; here and in what follows, we abbreviate

$$
u_{i}^{N}:=u\left(t_{i}^{N}\right), \quad \dot{u}_{i}^{N}:=\dot{u}\left(t_{i}^{N}\right), \quad \lambda_{i}^{N}:=\lambda\left(t_{i}^{N}\right) \quad \text { for all } i \in\{1, \ldots N\}
$$

Then we will exploit convexity inequalities for $\mathrm{W}_{2}, \mathrm{~W}_{p}$ and $\mathcal{I}_{\mathrm{C}}$. Let us point out that the semistability condition is valid for all $t \in[0, T]$, whereas the momentum balance (3.29a) holds only for almost every $t \in(0, T)$. Hence, the sequence of partitions $\left(\tau_{N}\right)_{N}$ with $\tau_{N} \rightarrow 0$ as $N \rightarrow \infty$ has to be carefully chosen such that (4.27) holds for every $t_{i}^{N}$ involved.

Proposition 4.7 (Upper estimate for the mechanical energy).

Let $k \in \mathbb{N}$ be fixed. Let (3.7), (3.8), (3.12), and (3.14) hold true. Let $(u, z, w)$ be an energetic solution to the SBV-adhesive system. Then the mechanical energy inequality (3.31) also holds in the opposite direction, i.e. for all $t \in[0, T]$

$$
\Phi_{k}(u(t), z(t))+\int_{0}^{t} 2 \mathcal{R}_{2}(e(\dot{u})) \mathrm{d} s+\operatorname{Var}_{\mathcal{R}_{1}}(z ;[0, t]) \geq \Phi_{k}\left(u_{0}, z_{0}\right)+\int_{0}^{t} \int_{\Omega \backslash \Gamma} \Theta(w) \mathbb{B}: e(\dot{u}) \mathrm{d} x \mathrm{~d} s+\int_{0}^{t}\langle\mathrm{~F}, \dot{u}\rangle \mathrm{d} s .
$$

Hence, we have mechanical energy equality for the adhesive systems. 
Proof. For $t \in(0, T]$ fixed consider a sequence of partitions (4.26) with $\tau_{N} \rightarrow 0$ as $N \rightarrow \infty$, so that (4.27) is well-defined for all $N \in \mathbb{N}$. Observe that the momentum balance at time $t$ may not hold, but from (3.27a) it follows that both $\|e(u(t))\|_{L^{p}(\Omega \backslash \Gamma)}$ and $\|e(u(t))\|_{L^{2}(\Omega \backslash \Gamma)}$ are well-defined for all $t \in[0, T]$. We now treat the different terms arising from (4.27). Since $\mathrm{W}_{2}$ and $\mathrm{W}_{p}$ are convex, it is

$$
\begin{aligned}
& \sum_{i=1}^{N} \int_{\Omega \backslash \Gamma}\left(\mathrm{DW}_{2}\left(e\left(u_{i-1}^{N}\right)\right)+\mathrm{DW}_{p}\left(e\left(u_{i-1}^{N}\right)\right)\right): e\left(u_{i}^{N}-u_{i-1}^{N}\right) \mathrm{d} x \\
& \leq \sum_{i=1}^{N} \int_{\Omega \backslash \Gamma}\left(\mathrm{W}_{2}\left(e\left(u_{i}^{N}\right)\right)+\mathrm{W}_{p}\left(e\left(u_{i}^{N}\right)\right)-\mathrm{W}_{2}\left(e\left(u_{i-1}^{N}\right)\right)-\mathrm{W}_{p}\left(e\left(u_{i-1}^{N}\right)\right)\right) \mathrm{d} x \\
& =\int_{\Omega \backslash \Gamma}\left(\mathrm{W}_{2}(e(u(t)))+\mathrm{W}_{p}(e(u(t)))-\mathrm{W}_{2}\left(e\left(u_{0}\right)\right)-\mathrm{W}_{p}\left(e\left(u_{0}\right)\right)\right) \mathrm{d} x .
\end{aligned}
$$

For the right-hand side of (4.27) we obtain

$$
\sum_{i=1}^{N}\left\langle F_{i-1}^{N}, u_{i}^{N}-u_{i-1}^{N}\right\rangle=\sum_{i=1}^{N} \int_{t_{i-1}}^{t_{i}}\langle F(s), \dot{u}(s)\rangle+\underbrace{\left\langle F_{i-1}^{N}-F(s), \dot{u}(s)\right\rangle}_{\rightarrow 0},
$$

where the second term tends to 0 due to the regularity (3.12a) of $F$ and (4.6a) of $u$. For all $k \in \mathbb{N}$ we have that $\lambda_{i-1}^{N} \in \partial \mathcal{F}_{k}\left(u_{i-1}^{N}\right)$ is given by $\left\langle\lambda_{i-1}^{N}, v\right\rangle=\left\langle\ell_{i-1}^{N}, v\right\rangle+\int_{\Gamma} k z_{i-1}^{N} \llbracket u_{i-1}^{N} \rrbracket \cdot \llbracket v \rrbracket \mathrm{d} \mathcal{H}^{d-1}$ with $\ell_{i-1}^{N} \in \partial \mathcal{I}_{\mathrm{C}}\left(u_{i-1}^{N}\right)$. Exploiting the convexity of $\mathcal{I}_{\mathrm{C}}$ and that $\mathcal{I}_{\mathrm{C}}\left(u_{i-1}^{N}\right)=\mathcal{I}_{\mathrm{C}}\left(u_{i}^{N}\right)=0$ we find

$$
\begin{aligned}
& \sum_{i=1}^{N}\left\langle\ell_{i-1}^{N}, u_{i}^{N}-u_{i-1}^{N}\right\rangle+\sum_{i=1}^{N} \int_{\Gamma} k z_{i-1}^{N} \llbracket u_{i-1}^{N} \rrbracket \cdot \llbracket u_{i}^{N}-u_{i-1}^{N} \rrbracket \mathrm{d} \mathcal{H}^{d-1} \\
& \leq 0+\sum_{i=1}^{N} \int_{t_{i-1}^{N}}^{t_{i}^{N}} \int_{\Gamma} k z_{i-1}^{N} \llbracket u_{i-1}^{N} \rrbracket \cdot \llbracket \frac{u_{i}^{N}-u_{i-1}^{N}}{\tau_{N}} \rrbracket \mathrm{d} \mathcal{H}^{d-1} \mathrm{~d} s \\
& =\underbrace{\sum_{i=1}^{N} \int_{t_{i-1}^{N}}^{t_{i}^{N}} \int_{\Gamma} k z_{i-1}^{N} \llbracket u_{i-1}^{N} \rrbracket \cdot \llbracket \dot{u}_{i-1}^{N} \rrbracket \mathrm{d} \mathcal{H}^{d-1} \mathrm{~d} s}_{\downarrow}+\underbrace{\sum_{i=1}^{N} \int_{t_{i-1}^{N}}^{t_{i}^{N}} \int_{\Gamma} k z_{i-1}^{N} \llbracket u_{i-1}^{N} \rrbracket \cdot \llbracket \frac{u_{i}^{N}-u_{i-1}^{N}}{\tau_{N}}-\dot{u}_{i-1}^{N} \rrbracket \mathrm{d} \mathcal{H}^{d-1} \mathrm{~d} s}_{\downarrow} \\
& \int_{0}^{t} \int_{\Gamma} k z \llbracket u \rrbracket \cdot \llbracket \dot{u} \rrbracket \mathrm{d} \mathcal{H}^{d-1} \mathrm{~d} s
\end{aligned}
$$

where the convergence of the Riemann-sums is due to (4.6a) and (4.6c). To obtain that the second term on the right-hand side tends to 0 one uses that $\|z\|_{L^{\infty}} \leq 1$ and then applies Hölder's inequality in $L^{2}\left(0, t ; L^{2}\left(\Gamma ; \mathbb{R}^{d}\right)\right)$ together with

$$
\begin{aligned}
\sum_{i=1}^{N} \int_{t_{i-1}}^{t_{i}}\left\|\frac{u_{i}^{N}-u_{i-1}^{N}}{\tau_{N}}-\dot{u}_{i-1}^{N}\right\|_{W^{1,2}\left(\Omega \backslash \Gamma ; \mathbb{R}^{d}\right)}^{2} \mathrm{~d} s & =\sum_{i=1}^{N} \tau_{N}\left\|\frac{u_{i}^{N}-u_{i-1}^{N}}{\tau_{N}}\right\|_{W^{1,2}}^{2}+\tau_{N}\left\|\dot{u}_{i-1}^{N}\right\|_{W^{1,2}}^{2}-2 \tau_{N}\left\langle\frac{u_{i}^{N}-u_{i-1}^{N}}{\tau_{N}}, \dot{u}_{i-1}^{N}\right\rangle \\
& \rightarrow\|\dot{u}\|_{L^{2}\left(0, t ; W^{1,2}\right)}^{2}+\|\dot{u}\|_{L^{2}\left(0, t ; W^{1,2}\right)}^{2}-2\|\dot{u}\|_{L^{2}\left(0, t ; W^{1,2}\right)}^{2}=0,
\end{aligned}
$$

where the convergence of the Riemann-sums is due to (4.6a). 
For the term involving the viscous dissipation we have

$$
\begin{aligned}
& \sum_{i=1}^{N} \int_{\Omega \backslash \Gamma} \mathrm{DR}_{2}\left(e\left(\dot{u}_{i-1}^{N}\right)\right): e\left(u_{i}^{N}-u_{i-1}^{N}\right) \mathrm{d} x \\
& =\underbrace{\left.\sum_{i=1}^{N} \int_{\Omega \backslash \Gamma}\left(t_{i}^{N}-t_{i-1}^{N}\right) \mathrm{DR}_{2}\left(e\left(\dot{u}_{i-1}^{N}\right)\right): e\left(\dot{u}_{i-1}^{N}\right)\right) \mathrm{d} x}_{\downarrow}+\underbrace{\sum_{i=1}^{t} \int_{t_{i-1}^{N}}^{t_{i}^{N}} \int_{\Omega \backslash \Gamma} \operatorname{DR}_{2}\left(e\left(\dot{u}_{i-1}^{N}\right)\right): e\left(\frac{u_{i}^{N}-u_{i-1}^{N}}{\tau_{N}}-\dot{u}_{i-1}^{N}\right) \mathrm{d} x \mathrm{~d} s}_{\downarrow} \\
& \int_{0}^{\mathrm{DR}_{2}(e(\dot{u})): e(\dot{u}) \mathrm{d} x \mathrm{~d} s}
\end{aligned}
$$

where the convergence of the Riemann-sums is again due to (4.6a) and the convergence to 0 of the second term is obtained using (4.32). It remains to analyze the term involving the thermal stresses, i.e.

$$
\begin{aligned}
& \sum_{i=1}^{N} \int_{\Omega \backslash \Gamma}-\mathbb{B} \Theta_{i-1}^{N}: e\left(u_{i}^{N}-u_{i-1}^{N}\right) \mathrm{d} x \\
& =\underbrace{\sum_{t_{i-1}^{N}}^{t_{i}^{N}} \int_{\Omega \backslash \Gamma}-\mathbb{B}_{i-1}^{N}: e\left(\dot{u}_{i-1}^{N}\right) \mathrm{d} x \mathrm{~d} s}_{\int_{0}^{t} \int_{\Omega \backslash \Gamma}^{N}-\mathbb{B} \Theta(w): e(\dot{u}) \mathrm{d} x \mathrm{~d} s}+\underbrace{\sum_{i=1}^{N} \int_{t_{i-1}^{N}}^{t_{i}^{N}} \int_{\Omega \backslash \Gamma}-\mathbb{B} \Theta_{i-1}^{N}: e\left(\frac{u_{i}^{N}-u_{i-1}^{N}}{\tau_{N}}-\dot{u}_{i-1}^{N}\right) \mathrm{d} x \mathrm{~d} s}_{\downarrow} \\
& 0,
\end{aligned}
$$

where we exploited (4.6a), (4.6j), and again (4.32). Collecting (4.29)-(4.34) leads to

$$
\begin{aligned}
\int_{0}^{t}\langle F, \dot{u}\rangle \mathrm{d} s \leq \int_{\Omega \backslash \Gamma}\left(\mathrm{W}_{2}(e(u(t)))+\mathrm{W}_{p}(e(u(t)))-\mathrm{W}_{2}\left(e\left(u_{0}\right)\right)-\mathrm{W}_{p}\left(e\left(u_{0}\right)\right)\right) \mathrm{d} x \\
\quad+\int_{0}^{t} \int_{\Omega \backslash \Gamma} 2 \mathrm{R}_{2}(e(\dot{u}))-\mathbb{B} \Theta(w): e(\dot{u}) \mathrm{d} x \mathrm{~d} s+\int_{0}^{t} \int_{\Gamma} k z \llbracket u \rrbracket \cdot \llbracket \dot{u} \rrbracket \mathrm{d} \mathcal{H}^{d-1} \mathrm{~d} s
\end{aligned}
$$

Now, a similar estimate for the surface energy has to be established. As in [57] we therefore test the semistability inequality at time $t_{i-1}^{N}$ with $z_{i}^{N}$. Summing up over $i \in\{0, \ldots, N\}$ yields

$$
\begin{aligned}
& \sum_{i=1}^{N} \int_{\Gamma} \frac{k}{2} z_{i-1}^{N}\left|\llbracket u_{i-1}^{N} \rrbracket\right|^{2} \mathrm{~d} \mathcal{H}^{d-1}+\mathcal{G}_{\mathrm{b}}\left(z_{i-1}^{N}\right) \leq \sum_{i=1}^{N} \int_{\Gamma} \frac{k}{2} z_{i}^{N}\left|\llbracket u_{i-1}^{N} \rrbracket\right|^{2} \mathrm{~d} \mathcal{H}^{d-1}+\mathcal{G}_{\mathrm{b}}\left(z_{i}^{N}\right)+\mathcal{R}_{1}\left(z_{i}^{N}-z_{i-1}^{N}\right) \\
& =\sum_{i=1}^{N} \int_{\Gamma} \frac{k}{2} z_{i}^{N}\left|\llbracket u_{i}^{N} \rrbracket\right|^{2} \mathrm{~d} \mathcal{H}^{d-1}+\mathcal{G}_{\mathrm{b}}\left(z_{i}^{N}\right)+\mathcal{R}_{1}\left(z_{i}^{N}-z_{i-1}^{N}\right)+\sum_{i=1}^{N} \int_{\Gamma} \frac{k}{2} z_{i}^{N}\left(\left|\llbracket u_{i-1}^{N} \rrbracket\right|^{2}-\left|\llbracket u_{i}^{N} \rrbracket\right|^{2}\right) \mathrm{d} \mathcal{H}^{d-1} .
\end{aligned}
$$

Scooping the left-hand side to the right, exploiting the cancelation of redundant terms and using that the last term in (4.36) can be expressed via the chain rule, leads to

$$
\begin{gathered}
0 \leq \int_{\Gamma} \frac{k}{2} z(t)|\llbracket u(t) \rrbracket|^{2} \mathrm{~d} \mathcal{H}^{d-1}-\int_{\Gamma} \frac{k}{2} z_{0}\left|\llbracket u_{0} \rrbracket\right|^{2} \mathrm{~d} \mathcal{H}^{d-1}+\mathcal{G}_{\mathrm{b}}(z(t))-\mathcal{G}_{\mathrm{b}}\left(z_{0}\right)+\mathcal{R}_{1}\left(z(t)-z_{0}\right) \\
-\sum_{i=1}^{N} \int_{t_{i-1}^{N}}^{t_{i}^{N}} \int_{\Gamma} k z_{i}^{N} \llbracket u(s) \rrbracket \cdot \llbracket \dot{u}(s) \rrbracket \mathrm{d} \mathcal{H}^{d-1} \mathrm{~d} s .
\end{gathered}
$$


For the last term in (4.37) we calculate

$$
\begin{aligned}
& -\sum_{i=1}^{N} \int_{t_{i-1}^{N}}^{t_{i}^{N}} \int_{\Gamma} k z_{i}^{N} \llbracket u(s) \rrbracket \cdot \llbracket \dot{u}(s) \rrbracket \mathrm{d} \mathcal{H}^{d-1} \mathrm{~d} s \\
& \leq-\underbrace{\sum_{i=1}^{N} \int_{t_{i-1}^{N}}^{t_{i}^{N}} \int_{\Gamma} k z_{i}^{N} \llbracket u_{i}^{N} \rrbracket \cdot \llbracket \dot{u}_{i}^{N} \rrbracket \mathrm{d} \mathcal{H}^{d-1} \mathrm{~d} s}_{\downarrow}+\underbrace{\sum_{i=1}^{N} \int_{t_{i-1}^{N}}^{t_{i}^{N}} \int_{\Gamma} k z_{i}^{N}\left(\left|\llbracket u \rrbracket-\llbracket u_{i}^{N} \rrbracket\right|\left|\llbracket \dot{u}_{i}^{N} \rrbracket\right|+\left|\llbracket \dot{u} \rrbracket-\llbracket \dot{u}_{i}^{N} \rrbracket \| \llbracket u \rrbracket\right|\right) \mathrm{d} \mathcal{H}^{d-1} \mathrm{~d} s}_{\downarrow} \\
& -\int_{0}^{t} \int_{\Gamma} k z \llbracket u \rrbracket \cdot \llbracket \dot{u} \rrbracket \mathrm{d} \mathcal{H}^{d-1} \mathrm{~d} s \quad 0,
\end{aligned}
$$

where above the convergence of the Riemann-sums is due to $u \in W^{1,2}\left(0, t ; W_{\Gamma_{\mathrm{D}}}^{1,2}\left(\Omega \backslash \Gamma ; \mathbb{R}^{d}\right)\right)$ by (4.6a) and $z \in L^{\infty}((0, t) \times \Gamma)$ by (4.6c). Altogether we have obtained

$$
0 \leq \int_{\Gamma} \frac{k}{2} z(t) \llbracket u(t) \rrbracket^{2} \mathrm{~d} \mathcal{H}^{d-1}-\int_{\Gamma} \frac{k}{2} z_{0} \| \llbracket u_{0} \rrbracket^{2} \mathrm{~d} \mathcal{H}^{d-1}+\mathcal{G}_{\mathrm{b}}(z(t))-\mathcal{G}_{\mathrm{b}}\left(z_{0}\right)+\mathcal{R}_{1}\left(z(t)-z_{0}\right)-\int_{0}^{t} \int_{\Gamma} k z \llbracket u \rrbracket \cdot \llbracket \dot{u} \rrbracket \mathrm{d} \mathcal{H}^{d-1} \mathrm{~d} s .
$$

The bulk (4.35) and the surface (4.38) estimates yield the upper mechanical energy estimate (4.28) for the SBV-adhesive system.

The analog of Proposition 4.7 is obtained for the Modica-Mortola adhesive system, upon repeating the steps for the surface energy with the regularization $\mathcal{G}_{m}$ instead of $\mathcal{G}_{\mathrm{b}}$ in $(4.36)-(4.38)$.

We are now in the position to conclude the following

Corollary 4.8 (Enthalpy and total energy equality).

Let the assumptions of Proposition 4.7 hold. Let $(u, z, w)$ be an (approximable) energetic solution to the SBV-adhesive contact system (cf. Thm. 4.3). Then the enthalpy (3.33) and the total energy (3.35) estimates hold as equalities for $(u, z, w)$.

Proof. First of all, we deduce from the mechanical energy equality the convergence of the viscous dissipation, i.e.

$$
\int_{0}^{t} 2 \mathcal{R}_{2}\left(e\left(\dot{u}_{m}\right)\right) \mathrm{d} s \rightarrow \int_{0}^{t} 2 \mathcal{R}_{2}(e(\dot{u})) \mathrm{d} s
$$

where $\left(u_{m}, z_{m}, w_{m}\right)$ are the solutions of the Modica-Mortola adhesive systems and $(u, z, w)$ is the solution of the SBV-adhesive system. Indeed, arguing as in $[54,57]$ we develop the chain of inequalities (4.40) below. There, the first inequality is obtained by lower semicontinuity and convergences (4.6a), (4.6c), while the second one relies on the mechanical energy equality for the Modica-Mortola adhesive systems. The third equality is due to $u_{m} \rightarrow u$ strongly in $L^{p}\left(0, T ; W^{1, p}\left(\Omega \backslash \Gamma ; \mathbb{R}^{d}\right)\right)$ by Theorem 4.5 , assumption (4.5) and convergence (4.6j), while the mechanical energy equality for the SBV-adhesive systems is exploited for the last equality.

$$
\begin{aligned}
& \int_{0}^{t} 2 \mathcal{R}_{2}(e(\dot{u})) \mathrm{d} s+\operatorname{Var}_{\mathcal{R}_{1}}(z ;[0, t]) \leq \liminf _{m \rightarrow \infty} \int_{0}^{t} 2 \mathcal{R}_{2}\left(e\left(\dot{u}_{m}\right)\right) \mathrm{d} s+\operatorname{Var}_{\mathcal{R}_{1}}\left(z_{m} ;[0, t]\right) \\
& \leq \limsup _{m \rightarrow \infty} \Phi_{m, k}\left(u_{m}^{0}, z_{m}^{0}\right)-\Phi_{m, k}\left(u_{m}(t), z_{m}(t)\right)+\int_{0}^{t} \int_{\Omega \backslash \Gamma} \Theta\left(w_{m}\right) \mathbb{B}: e\left(\dot{u}_{m}\right) \mathrm{d} x \mathrm{~d} s+\int_{0}^{t}\left\langle F, \dot{u}_{m}\right\rangle \mathrm{d} s \\
& =\Phi_{k}(u(0), z(0))-\Phi_{k}(u(t), z(t))+\int_{0}^{t} \int_{\Omega \backslash \Gamma} \Theta(w) \mathbb{B}: e(\dot{u}) \mathrm{d} x \mathrm{~d} s+\int_{0}^{t}\langle F, \dot{u}\rangle \mathrm{d} s \\
& =\int_{0}^{t} 2 \mathcal{R}_{2}(e(\dot{u})) \mathrm{d} s+\operatorname{Var}_{\mathcal{R}_{1}}(z ;[0, t]) .
\end{aligned}
$$


Since $\operatorname{Var}_{\mathcal{R}_{1}}\left(z_{m} ;[0, t]\right) \rightarrow \operatorname{Var}_{\mathcal{R}_{1}}(z ;[0, t])$ by (4.6c), from (4.40) we deduce the convergence (4.39) of the viscous dissipation, as well as (4.14).

Combining (4.14) and (4.39) with convergences (4.6a), (4.6c) and (4.6j) allows us to pass with $m \rightarrow \infty$ in the weak enthalpy equality of the Modica-Mortola adhesive systems and to obtain that the limit, i.e. the respective relation for the SBV-adhesive system, again is an equality. Finally, the total energy equality for the SBV-adhesive system is deduced by summing up the mechanical energy and the enthalpy equality.

While the analog of Corollary 4.8 holds for the Modica-Mortola adhesive contact system, for the brittle delamination system, however, our methods to gain energy equalities fail in the very first step, as the following remark highlights.

Remark 4.9 (Failure of the methods in the brittle setting).

As described along with (4.27), we have to avoid the occurrence of $\dot{u}$ in nonlinear, $p$-dependent terms due to a lack of regularity. In the adhesive setting we therefore test the momentum inclusion at time $t_{i-1}^{N}$ by $u\left(t_{i}^{N}\right)-u\left(t_{i-1}^{N}\right)$ and exploit convexity inequalities for $\mathrm{W}_{p}$ and $\mathcal{I}_{\mathrm{C}}$, cf. estimates (4.29) and (4.31). For the analogue of (4.31) in the brittle setting, one would have to estimate the term $\left\langle l_{i-1}^{N}, u\left(t_{i}^{N}\right)-u\left(t_{i-1}^{N}\right)\right\rangle$ with $l_{i-1}^{N} \in \partial \mathcal{J}_{\infty}\left(u_{i-1}^{N}, z\left(t_{i-1}^{N}\right)\right)$. This cannot be done by convexity inequalities because $z\left(t_{i-1}^{N}\right) \llbracket u\left(t_{i}^{N}\right) \rrbracket \neq 0$ is not excluded a.e. on $\Gamma$, therefore $\mathcal{J}_{\infty}\left(u_{i}^{N}, z\left(t_{i-1}^{N}\right)\right)=\infty$ is possible. Clearly this problem does not occur in the adhesive setting.

\section{From SBV-Adhesive Contact to SBV-Brittle Delamination}

In this section we deduce the existence of energetic solutions for the SBV-brittle delamination systems. This will be done by passing to the brittle limit $k \rightarrow \infty$ with the SBV-adhesive contact systems.

During the limit passage as $k \rightarrow \infty$ the properties of the surface energy functionals $\mathcal{F}_{k}$ from (3.40) change dramatically: their smooth contributions $\mathcal{J}_{k}\left(\cdot, z_{k}\right)$ for adhesive contact from (3.39) are supposed to approximate the nonsmooth functionals $\mathcal{J}_{\infty}(\cdot, z)$ for the brittle constraint from (3.43). In addition, also a suitable convergence of their functional derivatives is required in order to pass to the limit in the weak formulation of the momentum balance, see (3.29a) and (3.29b), respectively.

Testing the adhesive momentum balance (3.29a) with functions suited for the brittle equation (3.29b), i.e. functions in the set $\mathcal{U}_{z(t)}$ from (3.25), would need

$$
\text { for all } v \in \mathcal{U}_{z(t)}: \quad \int_{\Gamma} k z_{k}(t) \llbracket u_{k}(t) \rrbracket \cdot \llbracket v \rrbracket \mathrm{d} \mathcal{H}^{d-1} \stackrel{!}{\longrightarrow} 0 \quad \text { as } k \rightarrow \infty
$$

for a.a. $t \in(0, T)$, where $\left(u_{k}, z_{k}, w_{k}\right)_{k}$ are the SBV-adhesive solutions suitably converging to a limit $(u, z, w)$. But as we only have that $\int_{\Gamma} k z_{k}(t)\left|\llbracket u_{k}(t) \rrbracket\right|^{2} \mathrm{~d} \mathcal{H}^{d-1} \leq C$, while $\int_{\Gamma} z_{k}(t)|\llbracket v \rrbracket|^{2} \mathrm{~d} \mathcal{H}^{d-1} \rightarrow 0$ only without the prefactor $k$, the integral in (5.1) might even blow up to $\infty$. Hence, we have to avoid dealing with (5.1), i.e. passing to the limit in (3.29a) with fixed test functions $v \in \mathcal{U}_{z(t)}$. Instead, we intend to construct a suitable recovery sequence $\left(v_{k}\right)_{k}$ for the test functions $v \in \mathcal{U}_{z(t)}$, which satisfies

$$
\mathcal{J}_{k}\left(v_{k}, z_{k}(t)\right)=\int_{\Gamma} \frac{k}{2} z_{k}(t)\left|\llbracket v_{k} \rrbracket\right|^{2} \mathrm{~d} \mathcal{H}^{d-1}=0 \quad \text { for all } k \in \mathbb{N} \text { and for all } t \in[0, T] .
$$

Additionally $\left(v_{k}\right)_{k}$ has to feature a convergence suited to recover the bulk terms. In other words, for every $k \in \mathbb{N}, v$ has to be modified in such a way that the support of $\llbracket v_{k} \rrbracket$ fits to the null set of $z_{k}$ and, as $k \rightarrow \infty$, also $v_{k} \rightarrow v$ suitably in the bulk. For obvious reasons, this convergence necessitates that the supports of $z_{k}$ converge to the support of $z$ in the sense that, for a.a. $t \in(0, T)$ it holds

$$
\operatorname{supp} z_{k}(t) \subset \operatorname{supp} z(t)+B_{\rho(k, t)}(0) \text { for all } k \in \mathbb{N} \text { and } \rho(k, t) \rightarrow 0 \text { as } k \rightarrow \infty,
$$

where $B_{\rho(k, t)}(0)$ is the open ball around 0 of radius $\rho(k, t)$. The above inclusion has to be understood as $\mathcal{H}^{d-1}\left(\operatorname{supp} z_{k}(t) \backslash\left(\operatorname{supp} z(t)+B_{\rho(k, t)}(0)\right)\right)=0$. This so-called support convergence cannot be deduced from the 
convergence of functions in a particular metric. It is rather a fine property of sequences being semistable for the perimeter functional, as we will establish in Section 6.

Nonetheless, apart from this, the convergence of the bulk terms requires $v_{k} \rightarrow v$ strongly in the respective Sobolev space over the domain $\Omega_{-} \cup \hat{M} \cup \Omega_{+}$with $\hat{M}=\operatorname{supp} z(t)$. The strong convergence of the recovery sequence can be gained from a result in [42], which, for general $\hat{M}$ (of bad regularity), is only valid in $W^{1, p}\left(\Omega_{-} \cup\right.$ $\hat{M} \cup \Omega_{+} ; \mathbb{R}^{d}$ ) with $p>d$. This is the ultimate reason for the regularization of $p$-growth in the bulk energy. Like in Section 4.1, we cannot directly pass to the limit with the term of $p$-growth in the momentum inequality (3.29a), i.e. here with $\int_{\Omega \backslash \Gamma} \mathrm{DW}_{p}\left(e\left(u_{k}(t)\right): e\left(v_{k}-u_{k}(t)\right) \mathrm{d} x\right.$, as we again have to identify the weak limit of the sequence $\left(\mathrm{DW}_{p}\left(e\left(u_{k}\right)\right)\right)_{k}$. In fact, we will rather use the construction of $\left(v_{k}\right)_{k}$ to show that the sequence of functionals $\left(\mathcal{F}_{k}\right)_{k}$ from (3.40) Mosco-converges to the functional $\mathcal{F}_{\infty}$ from (3.44), cf. Proposition 5.4 in Section 5.1. This will allow us to conclude convergence in the sense of graphs of the corresponding maximal monotone subdifferential operators and hence, to carry out the limit passage in the equivalent subdifferential reformulation (3.42). For the reader's convenience, we recall the following definition (see e.g. [5], Sect. 3.3, p. 295).

Definition 5.1 (Mosco-convergence).

Let $X$ be a Banach space and consider the (proper) functionals $F_{k}: X \rightarrow \mathbb{R}_{\infty}$, and $F: X \rightarrow \mathbb{R}_{\infty}$. We say that the sequence $\left(F_{k}\right)_{k}$ Mosco-converges as $k \rightarrow \infty$ to the functional $F$, if the following two conditions hold:

- lim inf inequality: for every $u \in X$ and $\left(u_{k}\right)_{k} \subset X$ there holds

$$
u_{k} \rightarrow u \text { weakly in } X \Rightarrow \liminf _{k \rightarrow \infty} F_{k}\left(u_{k}\right) \geq F(u) ;
$$

- $\lim$ sup inequality: for every $v \in X$ there exists a sequence $\left(v_{k}\right)_{k} \subset X$ such that

$$
v_{k} \rightarrow v \text { strongly in } X \text { and } \limsup _{k \rightarrow \infty} F_{k}\left(v_{k}\right) \leq F(v) .
$$

A closely related concept is the one of graph convergence of a sequence of maximal monotone operators: by ([5], p. 373, Thm. 3.66), Mosco-convergence of lower semicontinuous and convex functionals implies the one of the corresponding subdifferential operators. We recall (see [5], p. 360, Def. 3.58) that, given $A_{k}, A: X \rightrightarrows X^{*}$ (set-valued) maximal monotone operators defined on a Banach space $X$,

$$
\left(A_{k}\right)_{k} \text { converges in the sense of graphs in } X \text { to } A \Leftrightarrow\left\{\begin{array}{l}
\forall\left(u, u^{*}\right) \in X \times X^{*} \text { with } u^{*} \in A(u), \\
\exists\left(u_{k}, u_{k}^{*}\right)_{k} \subset X \times X^{*} \text { with } u_{k}^{*} \in A_{k}\left(u_{k}\right): \\
\left(u_{k}, u_{k}^{*}\right) \rightarrow\left(u, u^{*}\right) \text { strongly in } X \times X^{*} .
\end{array}\right.
$$

After outlining the features of our approach, let us now state the main result of this paper.

Theorem 5.2 (Adhesive contact approximation of SBV-brittle delamination).

Assume (3.7), (3.8) and (3.12). Let $\left(u_{k}, w_{k}, z_{k}\right)_{k}$ be a sequence of approximable solutions of the SBV-adhesive contact system, supplemented with initial data $\left(u_{k}^{0}, \theta_{k}^{0}, z_{k}^{0}\right)_{k}$ fulfilling (3.14) and (4.1). Suppose that, as $k \rightarrow \infty$

$$
\begin{aligned}
& u_{k}^{0} \rightarrow u_{0} \quad \text { in } W^{1, p}\left(\Omega \backslash \Gamma ; \mathbb{R}^{d}\right), \quad \theta_{k}^{0} \rightarrow \theta_{0} \quad \text { in } L^{\omega_{1}}(\Omega), \quad z_{k}^{0} \stackrel{*}{*} z_{0} \quad \text { in } L^{\infty}(\Gamma), \text { and } \\
& \Phi_{k}\left(u_{k}^{0}, z_{k}^{0}\right) \rightarrow \Phi_{\mathrm{b}}\left(u_{0}, z_{0}\right) .
\end{aligned}
$$

Then, there exist a (not relabeled) subsequence, and a triple $(u, w, z)$, such that convergences (4.6) hold for $\left(u_{k}, w_{k}, z_{k}\right)$ as $k \rightarrow \infty$ and $(u, w, z)$ is an energetic solution to the SBV-brittle delamination system, fulfilling the semistability condition (3.30) for all $t \in[0, T]$. In addition we have that

$$
u_{k} \rightarrow u \text { in } L^{p}\left(0, T ; W^{1, p}\left(\Omega \backslash \Gamma ; \mathbb{R}^{d}\right)\right) \quad \text { and } \quad \Phi_{k}\left(u_{k}, z_{k}\right) \rightarrow \Phi_{\mathrm{b}}(u, z) .
$$

Furthermore, the positivity property (4.2) holds. 
Proof. The proof follows the scheme outlined in Section 3.5.

Step 0. Selection of converging subsequences. For the sequence $\left(u_{k}, w_{k}, z_{k}\right)_{k}$, estimates (3.48)-(3.53) are valid and thus convergences (4.6) can be obtained in the very same way as in the proof of Theorem 4.3. Furthermore, notice that

$$
\sup _{k \in \mathbb{N}} \sup _{t \in[0, T]} \Phi_{k}\left(u_{k}(t), z_{k}(t)\right) \leq C \Rightarrow \frac{k}{2} \int_{\Gamma} z_{k}(t)\left|\llbracket u_{k}(t) \rrbracket\right|^{2} \mathrm{~d} \mathcal{H}^{d-1} \leq C \text { for all } t \in[0, T], k \in \mathbb{N} .
$$

Now, it follows from (4.6b) via Sobolev trace theorems that $\llbracket u_{k} \rrbracket \rightarrow \llbracket u \rrbracket$ in $\mathrm{C}^{0}\left([0, T] ; \mathrm{C}^{0}\left(\Gamma ; \mathbb{R}^{d}\right)\right)$. Hence, we obtain $\llbracket u \rrbracket \cdot \mathrm{n} \geq 0$, and also taking into account $(4.6 \mathrm{~d})$ we find that $\int_{\Gamma} z_{k}(t)\left|\llbracket u_{k}(t) \rrbracket\right|^{2} \mathrm{~d} \mathcal{H}^{d-1} \rightarrow \int_{\Gamma} z(t)|\llbracket u(t) \rrbracket|^{2} \mathrm{~d} \mathcal{H}^{d-1}$ for all $t \in[0, T]$. Therefore, thanks to (5.10) we easily conclude that the limit pair $(u, z)$ fulfills the brittle constraint $z \llbracket u \rrbracket=0$ a.e. on $(0, T) \times \Gamma$.

The proof of Steps 1 and 2, momentum balance and semistability, will be carried out in Sections 5.1 and 5.2, respectively. The mechanical energy inequality (3.31) and the enthalpy inequality (3.33) can be obtained by the very same lower semicontinuity arguments as in Steps 3 and 4 of the proof of Theorem 4.3, and the same for the positivity of the temperature, that is why we do not repeat it.

\subsection{Step 1: limit passage in the momentum equation via recovery sequences}

In this section we pass from adhesive to brittle in the subdifferential formulations of the momentum balance. As already mentioned, this will be done with the aid of a recovery sequence $\left(v_{k}\right)_{k}$ for the test functions $v \in \mathcal{U}_{z(t)}$ of the brittle momentum balance, which has to satisfy (5.2). The construction of this recovery sequence relies on the following Proposition 5.3. It was developed in ([49], Cor. 4.10) in order to pass from (Sobolev-) gradient delamination to Griffith-type delamination in the rate-independent setting. Its proof is based on a Hardy inequality derived in ([42], p. 190), which requires $p>d$.

In this section we will often indicate that $x=\left(x_{1}, y\right) \in \Omega$ is composed of the $x_{1}$-component and $y:=$ $\left(x_{2}, \ldots, x_{d}\right) \subset \mathbb{R}^{d-1}$. Moreover, in view of assumption (3.7c), we suppose without loss of generality that $\Omega$ is rotated in such a way that the normal n on $\Gamma$ points in the $x_{1}$-direction. Furthermore, within the statement of Proposition 5.3 we denote by $(\rho)$ a family of functions $\rho:[0, T] \rightarrow[0, \operatorname{diam} \Gamma / 2]$. To avoid overburdening notation, we will not specify their dependence on a specific parameter, and accordingly simply write $\rho(t) \rightarrow 0$ for the convergence to zero of the family $(\rho(t))$. Later on, we will apply Proposition (5.3) to a sequence $\left(\rho_{k}\right)_{k}, c f$. (5.12). Finally, for simplicity in the notation of $\xi_{\rho}^{\hat{M}}$ and $v^{\rho}$ (cf. (5.11) below), we will often omit the $t$-dependence of $\rho$.

Proposition 5.3 (Recovery sequence for the test functions, [49], Cor. 2).

Keep $t \in[0, T]$ fixed and let $\rho:[0, T] \rightarrow[0, \operatorname{diam} \Gamma / 2]$. Let $z(t) \in L^{\infty}(\Gamma)$ and let $\hat{M}(t):=\operatorname{supp} z(t)$. Let $d_{\hat{M}}(t, x):=\min _{\hat{x} \in \hat{M}(t)}|x-\hat{x}|$ for all $x \in \overline{\Omega_{ \pm}}$. Let $v(t) \in W^{1, p}\left(\Omega_{-} \cup \hat{M}(t) \cup \Omega_{+} ; \mathbb{R}^{d}\right)$, with $p>d$, such that $v(t)=0$ on $\Gamma_{\mathrm{D}}$ in the trace sense. With $\xi_{\rho}^{\hat{M}}(t, x):=\min \left\{\frac{1}{\rho(t)}\left(d_{\hat{M}}(t, x)-\rho(t)\right)^{+}, 1\right\}$ set

$$
v^{\rho}\left(t, x_{1}, y\right):=v_{\text {sym }}\left(t, x_{1}, y\right)+\xi_{\rho}^{\hat{M}}\left(t, x_{1}, y\right) v_{\text {anti }}\left(t, x_{1}, y\right)
$$

where $v_{\mathrm{sym}}\left(t, x_{1}, y\right):=\frac{1}{2}\left(v\left(t, x_{1}, y\right)+v\left(t,-x_{1}, y\right)\right)$ and $v_{\text {anti }}\left(t, x_{1}, y\right):=\frac{1}{2}\left(v\left(t, x_{1}, y\right)-v\left(t,-x_{1}, y\right)\right)$. Then, for a.a. $t \in(0, T)$ the following statements hold:

(i) $v^{\rho}(t) \rightarrow v(t)$ strongly in $W^{1, p}\left(\Omega_{-} \cup \Omega_{+} ; \mathbb{R}^{d}\right)$ for a family $(\rho(t))$ with $\rho(t) \rightarrow 0$,

(ii) $v(t) \in W^{1, p}\left(\Omega_{-} \cup \hat{M}(t) \cup \Omega_{+} ; \mathbb{R}^{d}\right) \Rightarrow v^{\rho}(t) \in W^{1, p}\left(\Omega_{-} \cup\left(\hat{M}(t)+B_{\rho(t)}(0)\right) \cup \Omega_{+} ; \mathbb{R}^{d}\right)$,

(iii) $\llbracket v(t) \rrbracket \cdot \mathrm{n} \geq 0$ on $\Gamma \Rightarrow \llbracket v^{\rho}(t) \rrbracket \cdot \mathrm{n} \geq 0$ on $\Gamma$.

We apply the construction of Proposition 5.3 to tailor a recovery sequence $\left(v_{k}\right)_{k}$ for any test function $v \in \mathcal{U}_{z(t)}$. For our purpose, the radii $\rho=\rho(k, t)$ in Proposition 5.3 are given by

$$
\rho(k, t):=\inf \left\{\rho>0: \operatorname{supp} z_{k}(t) \subset \operatorname{supp} z(t)+B_{\rho}(0)\right\} .
$$


As proved in the forthcoming Propositions 6.7 and 6.8 , we have $\rho(k, t) \rightarrow 0$ as $k \rightarrow \infty$ for all $t \in[0, T]$. Then, statement (ii) ensures that the sequence $\left(v_{k}\right)_{k}, v_{k}:=v^{\rho(k, t)}$, does not jump on $\operatorname{supp} z_{k}(t)$ for a.a. $t \in(0, T)$. Moreover, $\llbracket v^{\rho(k, t)} \rrbracket \cdot \mathrm{n} \geq 0$ on $\Gamma$ as given by statement $(i i i)$, while $(i)$ guarantees the desired convergence $v^{\rho(k, t)} \rightarrow v(t)$, only if $\rho(k, t) \rightarrow 0$ as $k \rightarrow \infty$. For $\operatorname{supp} z(t)=\emptyset$ this is shown in Proposition 6.7 and for $\operatorname{supp} z(t) \neq \emptyset$ in Proposition 6.8 .

The above recovery sequence will now be used to state the Mosco-convergence of several functionals involved in the adhesive momentum balance.

Proposition 5.4. Assume (3.7c).

(1) Let $\left(z_{k}\right)_{k} \subset \operatorname{SBV}(\Gamma ;\{0,1\})$, semistable for $\Phi_{k}\left(u_{k}(t), \cdot\right)$, with $z_{k} * z^{*}$ in $\operatorname{SBV}(\Gamma ;\{0,1\})$ as $k \rightarrow \infty$ and $X:=$ $W^{1, p}\left(\Omega \backslash \Gamma ; \mathbb{R}^{d}\right)$. Then, the functionals $\mathcal{J}_{k}\left(\cdot, z_{k}\right)(3.39)$ Mosco-converge in $X$ as $k \rightarrow \infty$ to $\mathcal{J}_{\infty}(\cdot, z)(3.43)$.

(2) Let the assumptions of (1) hold. Then, the sequence $\left(\mathcal{F}_{k}\left(\cdot, z_{k}\right)\right)_{k}(3.40)$ Mosco-converges in $X$ as $k \rightarrow \infty$ to $\mathcal{F}_{\infty}(\cdot, z)(3.44)$.

(3) Let $X:=L^{p}\left(0, T ; W^{1, p}\left(\Omega \backslash \Gamma ; \mathbb{R}^{d}\right)\right)$. For $z_{k}, z$ satisfying (4.6c) and any $t \in(0, T]$ consider the functionals

$$
\begin{array}{ll}
\widetilde{\mathcal{F}}_{k}\left(\cdot, z_{k}\right): X \rightarrow[0, \infty], & \widetilde{\mathcal{F}}_{k}\left(v, z_{k}\right):=\int_{0}^{t} \int_{\Omega \backslash \Gamma} \mathrm{W}_{p}(e(v(s))) \mathrm{d} x+\mathcal{F}_{k}\left(v(s), z_{k}(s)\right) \mathrm{d} s, \\
\widetilde{\mathcal{F}}_{\infty}(\cdot, z): X \rightarrow[0, \infty], & \widetilde{\mathcal{F}}_{\infty}(v, z):=\int_{0}^{t} \int_{\Omega \backslash \Gamma} \mathrm{W}_{p}(e(v(s))) \mathrm{d} x+\mathcal{F}_{\infty}(v(s), z(s)) \mathrm{d} s .
\end{array}
$$

Then the sequence $\left(\widetilde{\mathcal{F}}_{k}\left(\cdot, z_{k}\right)\right)_{k}$ Mosco-converges to the functional $\widetilde{\mathcal{F}}_{\infty}(\cdot, z)$ in $X$.

Proof. Ad (1). The liminf inequality (5.4) immediately follows from the fact that $\mathcal{J}_{k}\left(u_{k}, z_{k}\right) \geq 0$ for all $k \in \mathbb{N}$. This has to be combined with the observation that the limit pair $(u, z)$ fulfills $z \llbracket u \rrbracket=0$ on $\Gamma$, which can be checked arguing in the same way as throughout Step 0 of the proof of Theorem 5.2.

The limsup condition (5.5) is proved by associating with each $v \in W^{1, p}\left(\Omega \backslash \Gamma ; \mathbb{R}^{d}\right)$ s.t. $\mathcal{J}_{\infty}(v, z)<\infty$, i.e. $z \llbracket v \rrbracket=0$ on $\Gamma$, the recovery sequence

$$
v_{k}\left(x_{1}, y\right):= \begin{cases}v_{\mathrm{sym}}\left(x_{1}, y\right)+\xi_{\rho(k)}^{\operatorname{supp} z}\left(x_{1}, y\right) v_{\text {anti }}\left(x_{1}, y\right) & \text { if } \operatorname{supp} z \neq \emptyset \text { and } \operatorname{supp} z_{k} \not \subset \operatorname{supp} z \\ v\left(x_{1}, y\right) & \text { if } \operatorname{supp} z_{k} \subset \operatorname{supp} z \\ v\left(x_{1}, y\right) & \text { if } \operatorname{supp} z=\emptyset .\end{cases}
$$

For the non-trivial construction in the first line of (5.14) the radius $\rho(k)>0$ is defined by (5.12). If $\operatorname{supp} z_{k} \subset$ $\operatorname{supp} z$, it is $\rho(k)=0$ according to (5.12) and there is no need to modify $v$. The construction for the case $\operatorname{supp} z=\emptyset$ is due to Proposition 6.7 stating that, if $\operatorname{supp} z=\emptyset$, then also $\operatorname{supp} z_{k}=\emptyset$ from a particular index $k_{0}$ on. For $\operatorname{supp} z \neq \emptyset$ the construction is the one from Proposition 5.3. The sequence $\left(v_{k}\right)_{k}$ strongly converges to $v$ in $X$ by (i) of Proposition 5.3. From (ii) and (5.12) it follows that $z_{k} \llbracket v_{k} \rrbracket=0$ for every $k \in \mathbb{N}$, hence $\mathcal{J}_{k}\left(v_{k}, z_{k}\right)=\mathcal{J}_{\infty}(v, z)=0$ and $(5.5)$ is verified.

Clearly, (2) is an obvious consequence of (1), also taking into account that, the construction of the recovery sequence $\left(v_{k}\right)_{k}$ preserves the non-penetration constraint, $c f$. (iii) in Proposition 5.3.

Ad (3). Consider $v \in X=L^{p}\left(0, T ; W^{1, p}\left(\Omega \backslash \Gamma ; \mathbb{R}^{d}\right)\right)$. Again, the liminf inequality (5.4) is easy to check. As for the limsup inequality, for a.a. $s \in(0, t)$ fixed a recovery sequence for $v(s)=v\left(s, x_{1}, y\right)$ is given by $v_{k}(s)=v_{k}\left(s, x_{1}, y\right)$ from (5.14). We prove that $v_{k} \rightarrow v$ strongly in $X$. Statement $(i)$ of Proposition 5.3 yields that $v_{k}(s) \rightarrow v(s)$ strongly in $W^{1, p}\left(\Omega \backslash \Gamma ; \mathbb{R}^{d}\right)$, whence $\left\|v_{k}(s)\right\|_{W^{1, p}\left(\Omega \backslash \Gamma ; \mathbb{R}^{d}\right)} \rightarrow\|v(s)\|_{W^{1, p}\left(\Omega \backslash \Gamma ; \mathbb{R}^{d}\right)}$ pointwise a.e. in $(0, t)$. Moreover, due to $\xi_{\rho(k)}^{\hat{M}}(s, \cdot) \in[0,1]$ for a.a. $s \in(0, t)$, construction $(5.14)$ gives $\left\|v_{k}(s)\right\|_{W^{1, p}\left(\Omega \backslash \Gamma ; \mathbb{R}^{d}\right)} \leq$ $\|v(s)\|_{W^{1, p}\left(\Omega \backslash \Gamma ; \mathbb{R}^{d}\right)}$ with $\|v(\cdot)\|_{W^{1, p}\left(\Omega \backslash \Gamma ; \mathbb{R}^{d}\right)} \in L^{p}(0, t)$. Due to the dominated convergence theorem we thus have $v_{k} \rightarrow v$ in $L^{p}\left(0, t ; W^{1, p}\left(\Omega \backslash \Gamma ; \mathbb{R}^{d}\right)\right)$. 
Now, we want to carry out the limit passage in the momentum balance from adhesive to brittle exploiting convergences (4.6). As in Section 4.1, we observe that, there exists $\mu \in L^{p^{\prime}}\left(0, T ; L^{p^{\prime}}(\Omega)\right)$ such that, up to the extraction of a further (not relabeled) subsequence there holds

$$
\mathrm{DW}_{p}\left(e\left(u_{k}\right)\right) \rightarrow \mu \quad \text { in } L^{p^{\prime}}\left(0, T ; L^{p^{\prime}}(\Omega)\right) .
$$

Furthermore, a comparison in the reformulation (3.42) of the adhesive momentum equation for $\left(u_{k}, w_{k}, z_{k}\right)_{k}$ yields a bound for the sequence $\left(\lambda_{k}\right)_{k} \subset L^{p^{\prime}}\left(0, T ; W^{1, p}\left(\Omega \backslash \Gamma ; \mathbb{R}^{d}\right)^{*}\right)$ such that $\lambda_{k}(t) \in \partial_{u} \mathcal{F}_{k}\left(u_{k}(t), z_{k}(t)\right)$ for almost all $t \in(0, T)$ and $\left(u_{k}, w_{k}, z_{k}, \lambda_{k}\right)$ fulfill (3.42). Therefore, up to a subsequence,

$$
\lambda_{k} \rightarrow \lambda \quad \text { in } L^{p^{\prime}}\left(0, T ; W^{1, p}\left(\Omega \backslash \Gamma ; \mathbb{R}^{d}\right)^{*}\right) .
$$

Convergences (5.15) and (5.16), combined with (4.6a)-(4.6h) allow us to show, as for (4.17), that the quintuple $(u, w, z, \mu, \lambda)$ for almost all $t \in(0, T)$ and all $v \in W^{1, p}\left(\Omega \backslash \Gamma ; \mathbb{R}^{d}\right)$ fulfills

$$
\int_{\Omega \backslash \Gamma}\left(\mathrm{DR}_{2}(e(\dot{u}(t)))+\mathrm{DW}_{2}(e(u(t)))-\mathbb{B} \Theta(w(t))+\mu(t)\right): e(v) \mathrm{d} x+\langle\lambda(t), v\rangle=\langle\mathrm{F}(t), v\rangle .
$$

Thus, to be able to conclude that (5.17) is the momentum inclusion for the SBV-brittle limit, as in Section 4.1 we have to identify the limits

$$
\mu(t)=\mathrm{DW}_{p}(e(u(t))) \text { and } \quad \lambda(t) \in \partial_{u} \mathcal{F}_{\infty}(u(t), z(t)) \quad \text { for a.a. } t \in(0, T) .
$$

For this, we exploit the Mosco-convergence of the functionals $\widetilde{\mathcal{F}}_{k}\left(\cdot, z_{k}\right)$ defined in (5.13): indeed, we will apply the following Lemma 5.5 to the graph-convergent sequence $\left(\partial_{u} \widetilde{\mathcal{F}}_{k}\left(\cdot, z_{k}\right)\right)_{k}$.

Lemma 5.5. Let $X$ be a reflexive Banach space and $\left(A_{k}\right)_{k}$ a sequence of maximal monotone operators $A_{k}$ : $X \rightrightarrows X^{*}$ which converge in the sense of graphs to a maximal monotone operator A. Then the following holds

$$
\left.\begin{array}{l}
\left(u_{k}, u_{k}^{*}\right) \in X \times X^{*} \text { with } u_{k}^{*} \in A_{k}\left(u_{k}\right), \\
u_{k} \rightarrow u \text { in } X, u_{k}^{*} \rightarrow u^{*} \text { in } X^{*}, \\
\limsup _{k \rightarrow \infty}\left\langle u_{k}^{*}, u_{k}\right\rangle_{X} \leq\left\langle u^{*}, u\right\rangle_{X}
\end{array}\right\} \Rightarrow \quad\left(u, u^{*}\right) \in X \times X^{*} \text { with } u^{*} \in A(u) \text {. }
$$

The proof can be retrieved from the lines of the proof of ([5], p. 361, Prop. 3.59).

We then obtain the following result on the limit passage in the momentum balance, where, as in Proposition 4.5, the identification (5.18) again implies the strong convergence of $\left(u_{k}\right)_{k}$ in $L^{p}\left(0, T ; W^{1, p}\left(\Omega \backslash \Gamma ; \mathbb{R}^{d}\right)\right)$.

Proposition 5.6 (Passage to the limit in the momentum equation as $k \rightarrow \infty$ ).

Assume (3.7), (3.8), (3.12), (3.14), and let $\left(u_{k}, w_{k}, z_{k}\right)_{k}$ be a sequence of energetic solutions to the SBV-adhesive contact systems, for which convergences (4.6) to a limit triple $(u, w, z)$ hold as $k \rightarrow \infty$. Then, $(u, w, z)$ satisfy the weak formulation (3.29b) of the momentum equation in the brittle case. In addition, there holds

$$
u_{k} \rightarrow u \text { strongly in } L^{p}\left(0, T ; W^{1, p}\left(\Omega \backslash \Gamma ; \mathbb{R}^{d}\right)\right) \text { and }\left(\Phi^{\mathrm{bulk}}\left(u_{k}\right)+\widetilde{\mathcal{F}}_{k}\left(u_{k}, z_{k}\right)\right) \rightarrow\left(\Phi^{\mathrm{bulk}}(u)+\widetilde{\mathcal{F}}_{\infty}(u, z)\right) .
$$

Proof. To prove (5.18) we are going to show that $u^{*} \in X^{*}$ (with $X:=L^{p}\left(0, T ; W^{1, p}\left(\Omega \backslash \Gamma ; \mathbb{R}^{d}\right)\right)$ ) given by $\left\langle u^{*}, v\right\rangle_{X}:=\int_{0}^{t} \int_{\Omega} \mu(s): e(v(s)) \mathrm{d} x+\langle\lambda(s), v(s)\rangle \mathrm{d} s$ is such that $u^{*} \in \partial_{u} \widetilde{\mathcal{F}}_{\infty}(u, z)$. To this aim, we observe that the sequence $\left(u_{k}^{*}\right)_{k} \subset X^{*}$ defined by $\left\langle u_{k}^{*}, v\right\rangle_{X}:=\int_{0}^{t} \int_{\Omega} \mathrm{DW}_{p}\left(e\left(u_{k}(s)\right)\right): e(v(s)) \mathrm{d} x+\left\langle\lambda_{k}(s), v(s)\right\rangle \mathrm{d} s$ fulfills $u_{k}^{*} \in \partial_{u} \widetilde{\mathcal{F}}_{k}\left(u_{k}, z_{k}\right)$ and $u_{k}^{*} \rightarrow u^{*}$ in $X^{*}$. Then, we apply Lemma 5.5 to the sequence of maximal monotone subdifferential operators $\left(A_{k}\right)_{k}$ given by $A_{k}:=\partial_{u} \widetilde{\mathcal{F}}_{k}\left(\cdot, z_{k}\right): X \rightrightarrows X^{*}$ and verify the lim sup-estimate in (5.19). 
For this, we again test (3.42) by $u_{k}$, integrate in time, and take the $\lim \sup _{k \rightarrow \infty}$. Thus, the very same calculations as throughout (4.20) give

$$
\limsup _{k \rightarrow \infty} \int_{0}^{t}\left(\int_{\Omega \backslash \Gamma} \mathrm{DW}_{p}\left(e\left(u_{k}\right)\right): e\left(u_{k}\right) \mathrm{d} x+\left\langle\lambda_{k}, u_{k}\right\rangle\right) \mathrm{d} s \leq \int_{0}^{t}\left(\int_{\Omega \backslash \Gamma} \mu: e(u) \mathrm{d} x+\langle\lambda, u\rangle\right) \mathrm{d} s .
$$

Hence, $u^{*} \in \partial_{u} \widetilde{\mathcal{F}}_{\infty}(u, z)$ and we conclude (5.18) as in the proof of Proposition 4.5.

For the convergence of the energies in (5.20) it has to be shown that $\mathcal{J}_{k}\left(u_{k}, z_{k}\right) \rightarrow 0$. This is obtained by testing the adhesive momentum inequality (3.29a) by the recovery sequence $\left(v_{k}\right)_{k}$ constructed via (5.11) for the brittle limit solution $u$. Rearranging the terms in (3.29a), and exploiting that $z_{k} \llbracket v_{k} \rrbracket=0$ a.e. on $\Gamma$ by construction, yields for a.a. $t \in(0, T)$ that

$$
\begin{aligned}
0 \leq \int_{\Gamma} k z_{k}\left|\llbracket u_{k} \rrbracket\right|^{2} \mathrm{~d} x \leq & \int_{\Omega \backslash \Gamma}\left(\mathrm{DR}_{2}\left(e\left(\dot{u}_{k}\right)\right)+\mathrm{DW}_{2}\left(e\left(u_{k}\right)\right)-\mathbb{B} \Theta\left(w_{k}\right)+\mathrm{DW}_{p}\left(e\left(u_{k}\right)\right)\right): e\left(v_{k}-u_{k}\right) \mathrm{d} x-\left\langle F, v_{k}-u_{k}\right\rangle \\
& \longrightarrow 0 \quad \text { as } k \rightarrow \infty,
\end{aligned}
$$

since both $v_{k} \rightarrow u$ and $u_{k} \rightarrow u$ strongly in $W^{1, p}\left(\Omega \backslash \Gamma ; \mathbb{R}^{d}\right)$ for a.a. $t \in(0, T)$. Hence $\int_{\Gamma} k z_{k}\left|\llbracket u_{k} \rrbracket\right|^{2} \mathrm{~d} x \rightarrow 0$ as $k \rightarrow \infty$ for a.a. $t \in(0, T)$.

\subsection{Step 2: closedness of semistable sets}

We now prove that the limit pair $(u, z)$ complies with the semistability condition (3.30) by constructing a mutual recovery sequence, $c f$. Section 4.2 , for the semistable sequence $\left(z_{k}\right)_{k} \subset L^{\infty}(0, T ; \operatorname{SBV}(\Gamma ;\{0,1\}))$ fulfilling (4.6c). This construction is carried out in Proposition 5.9 below. It uses notation from the theory of BV-spaces, which can be found in Appendix A.2, cf. in particular Definitions A.10 and A.11. In order to guarantee that $\mathcal{R}_{1}\left(\tilde{z}_{k}-z_{k}\right)<\infty$ for the mutual recovery sequence $\left(\tilde{z}_{k}\right)_{k}$, we would like to apply a construction similar to the one developed in [64] for Sobolev-gradients, which mainly consists of considering the minimum of the stable sequence and the test function $\tilde{z}$. To deal with the gradient terms one exploits a chain rule formula for Sobolev-functions and the Lipschitz continuous minimum function, $c f$. [44]. A corresponding chain rule formula for distributional derivatives, see [3], is more complicated to apply, as it also involves a kind of tangential differential. For our purposes however, the following Theorem 5.7 on the decomposability of BV-functions will provide an alternative construction that allows us to circumvent this general chain rule formula.

Theorem 5.7 ([4], Thm. 3.84, Decomposability of $B V$-functions).

Let $D \subset \mathbb{R}^{m}$. Let $v_{1}, v_{2} \in \mathrm{BV}(D)$ and let $E$ be a set of finite perimeter in $D$, with its reduced boundary $\mathfrak{F} E$ oriented by the generalized inner normal $\nu_{E}$. Let $v_{i \mathfrak{F} E}^{ \pm}$denote the traces on $\mathfrak{F} E \cap D$, which exist for $\mathcal{H}^{m-1}$-a.a. $x \in \mathfrak{F} E \cap D$ and $\mathcal{X}_{E}$ the characteristic function of the set $E$. Then

$$
w:=v_{1} \mathcal{X}_{E}+v_{2} \mathcal{X}_{D \backslash E} \in \mathrm{BV}(D) \text { if and only if } \int_{\mathfrak{F} E \cap D}\left|v_{1_{\mathfrak{F} E}}^{+}-v_{2_{\mathfrak{F} E}}^{-}\right| \mathrm{d} \mathcal{H}^{m-1}<\infty .
$$

If $w \in \mathrm{BV}(D)$ then the measure $\mathrm{D} w$ is represented by

$$
\mathrm{D} w:=\mathrm{D} v_{1}\left\lfloor E^{1}+\mathrm{D} v_{2}\left\lfloor E^{0}+\left(v_{1_{\mathfrak{F} E}}^{+}-v_{2_{\mathfrak{F} E}}^{-}\right) \nu_{E} \otimes \mathcal{H}^{m-1}\lfloor(\mathfrak{F} E \cap D),\right.\right.
$$

where $E^{1}$ and $E^{0}$ denote the measure-theoretic interior and exterior of $E$.

Since the three Radon-measures $\mathrm{D} v_{1}\left\lfloor E^{1}, \mathrm{D} v_{2}\left\lfloor E^{0}\right.\right.$, and $\left(v_{1_{\mathfrak{F} E}}^{+}-v_{2_{\mathfrak{F} E}}^{-}\right) \nu_{E} \otimes \mathcal{H}^{m-1}\lfloor(\mathfrak{F} E \cap D)$ in (5.23) have disjoint supports in $D$, we conclude that

$$
|\mathrm{D} w|(D)=\left|\mathrm{D} v_{1}\right|\left(E^{1}\right)+\left|\mathrm{D} v_{2}\right|\left(E^{0}\right)+\int_{\mathfrak{F} E \cap D}\left|\left(v_{1_{\mathfrak{F} E}}^{+}-v_{2_{\mathfrak{F} E}}^{-}\right)\right| \mathrm{d} \mathcal{H}^{m-1} .
$$

We then have the following result (see [62] for the proof). 
Lemma 5.8. Let $D \subset \mathbb{R}^{m}$ and $v \in \mathrm{BV}(D)$ with $a \leq v \leq b \mathcal{H}^{m}$-a.e. in $D$ for constants $a, b \in \mathbb{R}$. Assume that $\Gamma$ is a $\mathcal{H}^{m-1}$-rectifiable set oriented by $\nu$ and denote by $v_{\Gamma}^{ \pm}$the traces of $v$ on $\Gamma$. Then $a \leq v_{\Gamma}^{ \pm}(x) \leq b$ for $\mathcal{H}^{m-1}$-a.a. $x \in D$.

In the proof of the following result we will apply Theorem 5.7 and Lemma 5.8 with $D=\Gamma$ and $m=d-1$.

Proposition 5.9 (Passage to the limit in the semistability condition as $k \rightarrow \infty$ ).

Assume (3.8), (3.12), (3.14), and let $\left(u_{k}, z_{k}\right)_{k}$ be a sequence of energetic solutions to the SBV-adhesive contact system, for which convergences (4.6a)-(4.6f) hold as $k \rightarrow \infty$. Then, the limit pair $(u, z)$ fulfills the semistability condition (3.30) with the energy $\Phi_{\mathrm{b}}$.

Proof. To prove (3.30) with $\Phi_{\mathrm{b}}$, it is sufficient to show for a.a. $t \in(0, T)$

$$
\forall \tilde{z} \in \mathcal{Z}_{\mathrm{SBV}}: \quad \Phi_{\mathrm{b}}^{\mathrm{surf}}(\llbracket u(t) \rrbracket, z(t)) \leq \Phi_{\mathrm{b}}^{\mathrm{surf}}(\llbracket u(t) \rrbracket, \tilde{z})+\mathcal{R}_{1}(\tilde{z}-z(t)) .
$$

We will check (5.25) for $t \in(0, T)$ fixed, thus we will omit the variable $t$ from now on. We verify the following MRS-condition: Let $\left(z_{k}\right)_{k} \subset \operatorname{SBV}(\Gamma ;\{0,1\})$ be a semistable sequence for the energies $\left(\Phi_{k}\right)_{k}$, with $z_{k} \stackrel{*}{\rightarrow} z$ in $\operatorname{SBV}(\Gamma ;\{0,1\})$. Then, for all $\tilde{z} \in \mathcal{Z}$ there is a sequence $\left(\tilde{z}_{k}\right)_{k} \subset \operatorname{SBV}(\Gamma ;\{0,1\})$ so that

$$
\limsup _{k \rightarrow \infty}\left(\Phi_{k}\left(\llbracket u_{k} \rrbracket, \tilde{z}_{k}\right)-\Phi_{k}\left(\llbracket u_{k} \rrbracket, z_{k}\right)+\mathcal{R}_{1}\left(\tilde{z}_{k}-z_{k}\right)\right) \leq \Phi_{\mathrm{b}}(\llbracket u \rrbracket, \tilde{z})-\Phi_{\mathrm{b}}(\llbracket u \rrbracket, z)+\mathcal{R}_{1}(\tilde{z}-z) .
$$

In the proof of (5.26), we may suppose that $\tilde{z} \leq z$ a.e. in $\Gamma$, hence $\mathcal{R}_{1}(\tilde{z}-z)<\infty$. Indeed, if there exists a $\mathcal{H}^{d-1}$-measurable set $B \subset \Gamma$ with $\mathcal{H}^{d-1}(B)>0$ and $\tilde{z}>z$ on $B$, then $\mathcal{R}_{1}(\tilde{z}-z)=\infty$ and (5.26) trivially holds. To avoid trivial cases, we also suppose that $\Phi_{\mathrm{b}}(\llbracket u \rrbracket, \tilde{z})<\infty$, hence $0 \leq \tilde{z} \leq 1$ and $\tilde{z} \llbracket u \rrbracket=0$ a.e. on $\Gamma$. To construct a mutual recovery sequence we set

$$
\tilde{z}_{k}:=\tilde{z} \mathcal{X}_{A_{k}}+z_{k}\left(1-\mathcal{X}_{A_{k}}\right) \text {, where } A_{k}:=\left\{x \in \Gamma: 0 \leq \tilde{z}(x) \leq z_{k}(x)\right\}=:\left[0 \leq \tilde{z} \leq z_{k}\right] .
$$

With this choice we ensure that $0 \leq \tilde{z}_{k} \leq z_{k}$ a.e. in $\Gamma$. Note that $\Gamma \backslash A_{k}=\left[z_{k}<\tilde{z}\right]=\left[z_{k}=0\right] \cap[\tilde{z}=1]$. Since $z_{k}, \tilde{z} \in \operatorname{SBV}(\Gamma ;\{0,1\})$ are the characteristic functions of sets $Z_{k}, Z$ of uniformly bounded, finite perimeter, and relying on Proposition A.8, we find that

$$
\exists C>0 \forall k \in \mathbb{N}: \quad P\left(A_{k}, \Gamma\right)=P\left(\Gamma \backslash A_{k}, \Gamma\right) \leq P\left(Z_{k}, \Gamma\right)+P(Z, \Gamma) \leq C .
$$

Additionally, Lemma 5.8 implies that $\left|\tilde{z}-z_{k}\right| \leq 1,|\tilde{z}| \leq 1$ as well as $\left|z_{k}\right| \leq 1 \mathcal{H}^{d-2}$-a.e. on the respective reduced boundaries. Hence, Theorem 5.7 can be applied, yielding that $\tilde{z}_{k} \in \mathrm{BV}(\Gamma)$ for all $k \in \mathbb{N}$.

We now observe that, as $z_{k} \rightarrow z$ in $L^{1}(\Gamma)$ and $\tilde{z} \leq z$ a.e. in $\Gamma$, the definition (5.27) of $\tilde{z}_{k}$ yields that $\tilde{z}_{k} \rightarrow \tilde{z}$ a.e. in $\Gamma$. Now, since $\left(\tilde{z}_{k}\right)_{k}$ is bounded in $L^{\infty}(\Gamma)$ by construction, this pointwise convergence improves to $\tilde{z}_{k} \rightarrow \tilde{z}$ in $L^{q}(\Gamma)$ for all $1 \leq q<\infty$. Using that $0 \leq \tilde{z}_{k} \leq z_{k}$ a.e. on $\Gamma$, we have that

$$
\limsup _{k \rightarrow \infty} \frac{k}{2} \int_{\Gamma}\left(\tilde{z}_{k}-z_{k}\right)\left|\llbracket u_{k} \rrbracket\right|^{2} \mathrm{~d} \mathcal{H}^{d-1} \leq 0=\int_{\Gamma}\left(J_{\infty}(\llbracket u \rrbracket, \tilde{z})-J_{\infty}(\llbracket u \rrbracket, z)\right) \mathrm{d} \mathcal{H}^{d-1} .
$$

Hence, in order to conclude the limsup estimate (5.26), it remains to prove that

$$
\limsup _{k \rightarrow \infty}\left(\mathcal{G}_{\mathrm{b}}\left(\tilde{z}_{k}\right)-\mathcal{G}_{\mathrm{b}}\left(z_{k}\right)+\mathcal{R}_{1}\left(\tilde{z}_{k}-z_{k}\right)\right) \leq \limsup _{k \rightarrow \infty}\left(\mathcal{G}_{\mathrm{b}}\left(\tilde{z}_{k}\right)-\mathcal{G}_{\mathrm{b}}\left(z_{k}\right)\right)+\limsup _{k \rightarrow \infty} \mathcal{R}_{1}\left(\tilde{z}_{k}-z_{k}\right)
$$

and we estimate the different terms in (5.29) separately.

Due to $\tilde{z}_{k} \rightarrow \tilde{z}$ in $L^{1}(\Gamma)$ and the fact that $\tilde{z}_{k} \leq z_{k}$ for all $k \in \mathbb{N}$ by construction we conclude that $\mathcal{R}_{1}\left(\tilde{z}_{k}-z_{k}\right) \rightarrow$ $\mathcal{R}_{1}(\tilde{z}-z)$ as $k \rightarrow \infty$.

Thus, to deduce the estimate for $\mathcal{G}_{\mathrm{b}}$, it remains to show that

$$
\limsup _{k \rightarrow \infty}\left(\left|\mathrm{D} \tilde{z}_{k}\right|(\Gamma)-\left|\mathrm{D} z_{k}\right|(\Gamma)\right) \leq|\mathrm{D} \tilde{z}|(\Gamma)-|\mathrm{D} z|(\Gamma) .
$$


For this we recall that $\tilde{z}_{k}=\tilde{z} \mathcal{X}_{A_{k}}+z_{k}\left(1-\mathcal{X}_{A_{k}}\right)$ as well as $z_{k}=z_{k}\left(\mathcal{X}_{A_{k}}+\left(1-\mathcal{X}_{A_{k}}\right)\right)$ and we express their derivatives, i.e. the Radon measures $\mathrm{D} \tilde{z}_{k}$ and $\mathrm{D} z_{k}$, with the aid of formulae (5.23) and (5.27). Thus, by (5.24) we obtain

$$
\left|\mathrm{D} \tilde{z}_{k}\right|(\Gamma)=|\mathrm{D} \tilde{z}|\left(A_{k}^{1}\right)+\left|\mathrm{D} z_{k}\right|\left(A_{k}^{0}\right)+\int_{\mathfrak{F} A_{k} \cap \Gamma}\left|\tilde{z}^{+}-z_{k}^{-}\right| \mathrm{d} \mathcal{H}^{d-2},
$$

where we applied Theorem A.18, guaranteeing the existence of the traces $\tilde{z}_{k}^{ \pm}$on the different parts of the reduced boundaries, and (5.24) to justify the equality in (5.31). Similarly we find

$$
-\left|\mathrm{D} z_{k}\right|(\Gamma)=-\left|\mathrm{D} z_{k}\right|\left(A_{k}^{1}\right)-\left|\mathrm{D} z_{k}\right|\left(A_{k}^{0}\right)-\int_{\mathfrak{F} A_{k} \cap \Gamma}\left|z_{k}^{+}-z_{k}^{-}\right| \mathrm{d} \mathcal{H}^{d-2}
$$

We note that both $\left|\mathrm{D} \tilde{z}_{k}\right|\left(A_{k}^{0}\right)=0$ and $-\left|\mathrm{D} z_{k}\right|\left(A_{k}^{0}\right)=0$ in (5.31) and (5.32). We now prove that the boundary terms in $(5.31)+(5.32)$ can be estimated as follows for all $k \in \mathbb{N}$ :

$$
\int_{\mathfrak{F} A_{k} \cap \Gamma}\left|\tilde{z}^{+}-z_{k}^{-}\right| \mathrm{d} \mathcal{H}^{d-2}-\int_{\mathfrak{F} A_{k} \cap \Gamma}\left|z_{k}^{+}-z_{k}^{-}\right| \mathrm{d} \mathcal{H}^{d-2} \leq \int_{\mathfrak{F} A_{k} \cap \Gamma}\left|\tilde{z}^{+}-\tilde{z}^{-}\right| \mathrm{d} \mathcal{H}^{d-2} .
$$

To verify estimate (5.33) we use the information on the traces stated in Lemma 5.8 and distinguish between all possible relations. On $\mathfrak{F} A_{k} \cap \Gamma$ it holds $0 \leq \tilde{z}^{+} \leq z_{k}^{+}$and $0 \leq z_{k}^{-}<\tilde{z}^{-} \mathcal{H}^{d-2}$-a.e.. Hence, for $\mathcal{H}^{d-2}$-a.a. $x \in \mathfrak{F} A_{k} \cap \mathfrak{F}\left(\Gamma \backslash A_{k}\right) \cap \Gamma$ with

$$
\begin{array}{rll}
z_{k}^{+} \leq z_{k}^{-} \quad \text { it is } \quad \tilde{z}^{+} \leq z_{k}^{+} \leq z_{k}^{-}<\tilde{z}^{-}, \text {i.e. } & \left|\tilde{z}^{+}-z_{k}^{-}\right|<\left|\tilde{z}^{+}-\tilde{z}^{-}\right|, \\
z_{k}^{+}>z_{k}^{-} \quad \text { it is either } \quad \tilde{z}^{+} \leq z_{k}^{-}<z_{k}^{+} \leq \tilde{z}^{-}, \text {i.e. } & \left|\tilde{z}^{+}-z_{k}^{-}\right| \leq\left|\tilde{z}^{+}-\tilde{z}^{-}\right|, \\
\text {or } \quad \tilde{z}^{+} \leq z_{k}^{-}<\tilde{z}^{-} \leq z_{k}^{+}, \text {i.e. } & \left|\tilde{z}^{+}-z_{k}^{-}\right| \leq\left|\tilde{z}^{+}-\tilde{z}^{-}\right|, \\
\text {or } \quad z_{k}^{-}<\tilde{z}^{-} \leq \tilde{z}^{+} \leq z_{k}^{+} \text {, i.e. } & \left|\tilde{z}^{+}-z_{k}^{-}\right| \leq\left|z_{k}^{+}-z_{k}^{-}\right|, \\
\text {or } z_{k}^{-}<\tilde{z}^{+} \leq z_{k}^{+} \leq \tilde{z}^{-} \text {, i.e. } & \left|\tilde{z}^{+}-z_{k}^{-}\right| \leq\left|z_{k}^{+}-z_{k}^{-}\right|, \\
\text {or } z_{k}^{-}<\tilde{z}^{+}<\tilde{z}^{-} \leq z_{k}^{+}, \text {i.e. } & \left|\tilde{z}^{+}-z_{k}^{-}\right| \leq\left|z_{k}^{+}-z_{k}^{-}\right| .
\end{array}
$$

Using these estimates and denoting by $E$ the set of points, where one of the latter three relations holds, we find that

$$
\int_{\mathfrak{F} A_{k} \cap \Gamma}\left|\tilde{z}^{+}-z_{k}^{-}\right| \mathrm{d} \mathcal{H}^{d-2}-\int_{\mathfrak{F} A_{k} \cap \Gamma}\left|z_{k}^{+}-z_{k}^{-}\right| \mathrm{d} \mathcal{H}^{d-2} \leq \int_{\mathfrak{F} A_{k} \cap \Gamma \backslash E}\left|\tilde{z}^{+}-\tilde{z}^{-}\right| \mathrm{d} \mathcal{H}^{d-2}-0 \leq \int_{\mathfrak{F} A_{k} \cap \Gamma}\left|\tilde{z}^{+}-\tilde{z}^{-}\right| \mathrm{d} \mathcal{H}^{d-2} .
$$

Thus, (5.33) holds. In total we have obtained that the left-hand side of (5.30) can be estimated by

$$
\begin{aligned}
\limsup _{k \rightarrow \infty}\left(\left|\mathrm{D} \tilde{z}_{k}\right|(\Gamma)-\left|\mathrm{D} z_{k}\right|(\Gamma)\right) & \leq \limsup _{k \rightarrow \infty}\left(|\mathrm{D} \tilde{z}|\left(A_{k}^{1}\right)+\int_{\mathfrak{F} A_{k} \cap \Gamma}\left|\tilde{z}^{+}-\tilde{z}^{-}\right| \mathrm{d} \mathcal{H}^{d-2}-\left|\mathrm{D} z_{k}\right|\left(A_{k}^{1}\right)\right) \\
& \leq|\mathrm{D} \tilde{z}|(\Gamma)-\liminf _{k \rightarrow \infty}\left|\mathrm{D} z_{k}\right|\left(A_{k}^{1}\right) .
\end{aligned}
$$

Therefore, to establish (5.30) it remains to show that

$$
-\liminf _{k \rightarrow \infty}\left|\mathrm{D} z_{k}\right|\left(A_{k}^{1}\right) \leq-|\mathrm{D} z|(\Gamma) .
$$

To this aim, we first choose a (not relabeled) subsequence $\left(z_{k}\right)_{k}$ such that the lim inf is attained. Then, we introduce the sets $U_{n}:=\bigcup_{k=n}^{\infty}\left(\Gamma \backslash A_{k}\right)$. Since $\mathcal{H}^{d-1}\left(\Gamma \backslash A_{k}\right) \rightarrow 0$ as $k \rightarrow \infty$ we may choose a further subsequence s.t. $\sum_{k=1}^{\infty} \mathcal{H}^{d-1}\left(\Gamma \backslash A_{k}\right)<\infty$. Hence for this subsequence, $\mathcal{H}^{d-1}\left(U_{n}\right)<\infty$ and $\mathcal{H}^{d-1}\left(U_{n}\right) \rightarrow 0$ as $n \rightarrow \infty$. We set $\lim _{n \rightarrow \infty} U_{n}=N$ and put $\Gamma_{n}:=\Gamma \backslash U_{n}$, which satisfies $\Gamma_{n} \subset A_{k}$ for all $k \geq n$. Then, also $\Gamma_{n}^{1} \subset A_{k}^{1}$ as well as $\Gamma_{n}^{1} \subseteq \Gamma_{n+1}^{1} \subset \Gamma^{1}$ for all $n \in \mathbb{N}$ by Corollary A.12, 2. Since $\mathcal{H}^{d-1}(N)=0$ we conclude that $(\Gamma \backslash N)^{1}=\Gamma^{1}$ by Corollary A.12, 1 . Note that $\Gamma \subset \mathbb{R}^{d-1}$ is an open set, i.e. for all $x \in \Gamma$ there exists a 
constant $r_{x}>0$ such that $B_{r}(x) \subset \Gamma$ for all $r \leq r_{x}$. Hence $\Gamma^{1}=\Gamma$ and therefore $\left(\Gamma_{n}^{1}\right)_{n}$ satisfies $\Gamma_{n}^{1} \uparrow \Gamma$, i.e. $\Gamma_{1}^{1} \subseteq \ldots \subseteq \Gamma_{n}^{1} \subseteq \Gamma_{n+1}^{1} \subseteq \ldots \subseteq \Gamma=\cup_{n \in \mathbb{N}} \Gamma_{n}^{1}$. Keep $n \in \mathbb{N}$ fixed. Then the sets $\Gamma_{n}^{1} \subset A_{k}^{1}$ can be used to find a set independent of $k \geq n$, so that the lower semicontinuity of the total variation functional can be exploited on $\Gamma_{n}^{1}$ for the sequence $z_{k} \stackrel{*}{\rightarrow} z$ in $\operatorname{SBV}(\Gamma ;\{0,1\})$. Indeed, for all $k \geq n$ we have

$$
-\liminf _{k \rightarrow \infty}\left|\mathrm{D} z_{k}\right|\left(A_{k}^{1}\right) \leq-\liminf _{k \rightarrow \infty}\left|\mathrm{D} z_{k}\right|\left(\Gamma_{n}^{1}\right) \leq-|\mathrm{D} z|\left(\Gamma_{n}^{1}\right) \rightarrow-|\mathrm{D} z|(\Gamma) \text { as } n \rightarrow \infty,
$$

where the last convergence as $n \rightarrow \infty$ follows from $\Gamma_{n}^{1} \uparrow \Gamma$ and the $\sigma$-additivity of $|\mathrm{D} z|$. This finishes the proof of (5.35). Thus we conclude that the mutual recovery sequence $\left(\tilde{z}_{k}\right)_{k}$ given by $(5.27)$ satisfies the lim supestimate (5.26).

\section{Support PROPERTy of SEMISTABLE SEQUENCES}

We now investigate fine properties of the sequence $\left(z_{k}\right)_{k}$, which are exploited for proving the convergence of the momentum equation as $k \rightarrow \infty$ in Section 5.1. We will deduce such properties from the sole feature of semistability of the sequence $\left(z_{k}\right)_{k}$ with respect to the functionals $\Phi_{k}\left(u_{k}, \cdot\right)$.

The statement of the main result of this section, Theorem 6.1 below, is given for a generic sequence $\left(z_{k}\right)_{k} \subset L^{\infty}(0, T ; \operatorname{SBV}(\Gamma ;\{0,1\}))$ fulfilling the semistability condition (3.30). We refer to Remark 6.11 for further comments in this connection.

Theorem 6.1 (Support convergence). Assume (3.7c). Let $\left(z_{k}\right)_{k} \subset L^{\infty}(0, T ; \operatorname{SBV}(\Gamma ;\{0,1\}))$ fulfill semistability (3.30) for all $k \in \mathbb{N}$. Suppose that

$$
z_{k}(t) \stackrel{*}{\rightarrow} z(t) \quad \text { in } \operatorname{SBV}(\Gamma ;\{0,1\}) \quad \text { for all } t \in[0, T]
$$

for some $z \in L^{\infty}(0, T ; \operatorname{SBV}(\Gamma ;\{0,1\}))$. Set

$$
\rho(k, t):=\inf \left\{\rho>0: \operatorname{supp} z_{k}(t) \subset \operatorname{supp} z(t)+B_{\rho}(0)\right\} \quad \text { for all } t \in[0, T] \text { and all } k \in \mathbb{N} .
$$

Then, for all $t \in[0, T]$ we have support convergence, i.e.

$$
\operatorname{supp} z_{k}(t) \subset \operatorname{supp} z(t)+B_{\rho(k, t)}(0) \quad \text { and } \quad \rho(k, t) \rightarrow 0 \quad \text { as } k \rightarrow \infty .
$$

Note that convergence (6.3) is one part of Hausdorff convergence. Indeed, recall that, for any fixed $t \in[0, T]$ the sequence $\left(\operatorname{supp} z_{k}(t)\right)_{k}$ Hausdorff converges to $\operatorname{supp} z(t)$ if, in addition to $(6.3)$, we also have

$$
\exists(\tilde{\rho}(k, t))_{k}: \quad \operatorname{supp} z(t) \subset \operatorname{supp} z_{k}(t)+B_{\tilde{\rho}(k, t)}(0) \quad \text { and } \quad \tilde{\rho}(k, t) \rightarrow 0 \text { as } k \rightarrow \infty .
$$

As we will see, (6.4) can be concluded directly from (6.1), so that we will obtain the Hausdorff convergence for the sequence of supports in Corollary 6.9.

Since the solutions $\left(z_{k}\right)_{k}$ of the thermal delamination problems satisfy the semistability (3.30) for all $t \in[0, T]$, hereafter in most of the arguments for proving Theorem 6.1 we will suppose $t \in(0, T)$ fixed and omit indicating the dependence of the functions and of the radii on $t$. Moreover, all the ensuing calculations only involve functions defined on the interface $\Gamma \subset \mathbb{R}^{d-1}$, hence we will use the abbreviation

$$
m:=d-1 .
$$

The main idea we will develop is the following: Thanks to the SBV-gradient term in the energies $\Phi_{k}\left(u_{k}, \cdot\right)$ and $\Phi_{\mathrm{b}}(u, \cdot)(c f .(3.19),(3.22))$, the delamination parameters $z_{k}, z$ in the adhesive and brittle SBV-models are characteristic functions $z_{k}, z \in \operatorname{SBV}(\Gamma ;\{0,1\})$ of sets $Z_{k}, Z \subset \Gamma$ with finite perimeter. Furthermore, since the bulk energy is independent of $z_{k}$ and since $\mathcal{J}_{k}\left(u_{k}, \tilde{z}\right) \leq \mathcal{J}_{k}\left(u_{k}, z_{k}\right)$ for all $\tilde{z} \leq z_{k}$, the semistability of 
$z_{k}$ for $\Phi_{k}\left(u_{k}, \cdot\right), k \in \mathbb{N} \cup\{\infty\}$, implies the semistability of the underlying set $Z_{k}$ for the energy term $\mathcal{S}(\cdot):=$ $\mathrm{b} P(\cdot, \Gamma)-a_{0} \mathcal{H}^{m}(\cdot)$, i.e.

$$
\mathcal{S}\left(Z_{k}\right) \leq \mathcal{S}(\tilde{Z})+\mathcal{R}_{1}\left(\tilde{z}-z_{k}\right) \quad \text { with } \quad \mathcal{S}(Z):=\mathrm{b} P(Z, \Gamma)-a_{0} \mathcal{H}^{m}(Z) .
$$

Therefore, in the following arguments we will confine ourselves to working with (6.5). In particular, from (6.5), we will deduce that stable sets satisfy the lower density estimate (6.6), which, in turn, will allow us to conclude the support convergence (6.3). Property (6.6) is a very weak type of regularity of sets, but which at least prevents a set from having outward cones. For open sets this notion of regularity was termed as property $\mathfrak{a}$ in the works by Campanato, see e.g. ([12], p. 177) or ([13], p. 138), and in this context also used in e.g. $[16,32,34,35]$. The lower density estimate, which we here prove for semistable sets of finite perimeter, is of the following form

$$
\begin{array}{lll}
\forall y \in \operatorname{supp} z_{k} \forall \rho_{\star} \in(0, R): & \mathcal{H}^{m}\left(Z_{k} \cap B_{\rho_{\star}}(y)\right) \geq \mathfrak{a}(\Gamma) \rho_{\star}^{m}, \\
\forall y \in \operatorname{supp} z_{k} \quad \forall \rho_{\star} \geq R: & \mathcal{H}^{m}\left(Z_{k} \cap B_{\rho_{\star}}(y)\right) \geq \mathfrak{a}(\Gamma) R^{m},
\end{array}
$$

where $R, \mathfrak{a}(\Gamma)>0$, given in (6.23), are constants depending only on $\Gamma, m$ and the parameters $\mathrm{b}, a_{0}, a_{1}$.

Let us remark that it well established, cf. e.g. $[4,14,33,43]$, that quasi-minimizers $E \subset \Gamma$ of the perimeter functional in $\Gamma$ satisfy lower density estimates of the form

$$
\exists R, \beta>0 \forall y \in \mathfrak{F} E \cap \Gamma, \forall r<R \text { s.th. } B_{r}(y) \subset \Gamma: \quad \mathcal{H}^{m}\left(E \cap B_{r}(y)\right) \geq \beta r^{m} .
$$

Observe that, conversely, the lower density estimate (6.6) does not impose the restriction that the balls have to be strictly contained in $\Gamma$. This feature of (6.6) is important since we will have to treat sequences $\left(z_{k}\right)_{k}$ with uniform $\rho_{\star}$ for all $k \in \mathbb{N}$ in order to conclude support convergence (6.3). This enhanced estimate relies on a family of isoperimetric inequalities relative to $\Gamma \cap B_{\rho_{\star}}(y)$ with a uniform isoperimetric constant, independent of $y$ and $\rho_{\star}, c f$. Theorem 6.3. Moreover, we do not prove it in the direct way as for the lower density results in $[33,43]$, but rather proceed by contradiction. Our method first reveals a general lower bound on the volume of (semistable) sets in dependence of $\Gamma$ and the parameters $a_{0}, a_{1}$, b. It prevents that a subset of $Z \cap B_{\rho_{\star}}(y)$ can be eliminated in such a way that the new surface created is smaller than the surface of the part cut off. From this, (6.6) is deduced for semistable sets in a second step.

Finally, let us mention that estimates of the type (6.6) were also proved in the context of image processing for the Mumford-Shah functional, see e.g. ([23], Lem. 3.14) or ([4], p. 351). However, there, it is established for the $(m-1)$-dimensional Hausdorff-measure of the jump sets of SBV-quasi-minimizers and the respective estimate is called a lower density estimate.

To prove support convergence (6.3) and the lower density estimate (6.6), we will resort to refined tools from geometric measure theory. Once again, we refer the reader for Appendix A.2 for the background.

Before starting with the proofs of Theorem 6.1 and (6.6), we first motivate heuristically the reasons why support convergence may fail in general and how the $\operatorname{SBV}(\Gamma ;\{0,1\})$-setting allows us to deduce the lower density estimate (6.6), as well as (6.3).

Preliminary considerations. First of all, one should be aware that elements $z \in \operatorname{SBV}(\Gamma ;\{0,1\})$ (or in general $z \in L^{1}(\Gamma)$ ) are given by equivalence classes of functions differing on $\mathcal{H}^{m}$ - null sets, only. Hence, in this setting, the support $\operatorname{supp} z$ and the null set $N_{z}$ are rather defined similarly to the context of measures ([18], p. 60) by

$$
\begin{aligned}
\operatorname{supp} z & :=\cap\left\{A \mid A \text { closed, } \mathcal{H}^{m}(Z \backslash A)=0\right\} \text { and } N_{z}:=\Gamma \backslash \operatorname{supp} z, \\
\text { where } Z & :=\{x \in \Gamma \mid z(x) \neq 0\} .
\end{aligned}
$$

This definition yields supp $z$ closed and $N_{z}$ open, and for continuous functions it coincides with the conventional definition. Further, observe that, for any $B \subset \mathbb{R}^{m}$ with $\mathcal{H}^{m}(B)>0$, denoting by $\mathcal{X}_{B}$ the characteristic function of $B$, there holds

$$
\mathcal{H}^{m}\left(B \cap B_{r}(y)\right)>0 \quad \text { for all } r>0 \text { and all } y \in \operatorname{supp} \mathcal{X}_{B} \text {. }
$$

Another consequence of (6.8) is the following result. 
Corollary 6.2. Let $z \in L^{1}(\Gamma)$. Then $\operatorname{supp} z+B_{\rho(k)}(0) \rightarrow \operatorname{supp} z$ as $\rho(k) \rightarrow 0$, in the sense that $\mathcal{H}^{m}((\operatorname{supp} z+$ $\left.\left.B_{\rho(k)}(0)\right) \backslash \operatorname{supp} z\right) \rightarrow 0$ as $\rho(k) \rightarrow 0$.

Proof. First assume that $\operatorname{supp} z=\emptyset$. Then $\emptyset+B_{\rho(k)}(0)=\emptyset$ so that the statement holds true. Now, assume that $x \in \operatorname{supp} z+B_{\rho(k)}(0)$ for all $\rho(k)>0$. Then $x \notin N_{z}$, because $N_{z}$ is an open set. The thesis follows, observing that by monotonicity $\left(\mathcal{H}^{m}\left(N_{z} \cap\left(\operatorname{supp} z+B_{\rho(k)}(0)\right)\right)\right)_{k}$ converges to $\mathcal{H}^{m}\left(N_{z} \cap \cap_{k \in \mathbb{N}}\left(\operatorname{supp} z+B_{\rho(k)}(0)\right)\right)$ as $\rho(k) \rightarrow 0$.

While for every fixed $z \in L^{1}(\Gamma)$ we have $\operatorname{supp} z+B_{\rho(k)}(0) \rightarrow \operatorname{supp} z$ as $\rho(k) \rightarrow 0$, support convergence (6.3) is in general not true for arbitrary sequences $z_{k} \rightarrow z$ in $L^{1}(\Gamma)$ with supp $z \neq \emptyset$. Clearly, for any sequence $z_{k} \rightarrow z$ in $L^{1}(\Gamma)$, which can attain values in the whole interval $[0,1]$, there is a sequence $(\rho(k))_{k}$ with $\rho(k) \geq 0$ such that supp $z_{k} \subset \operatorname{supp} z+B_{\rho(k)}(0)$. This is due to the boundedness of $\Gamma$. But not necessarily $\rho(k) \rightarrow 0$ as $k \rightarrow \infty$, as can be seen from the following counterexample:

Example 1. Let $z=1$ on a closed set $Z \subset$ int $\Gamma$ and $z=0$ otherwise, and for all $k \in \mathbb{N}$ let $z_{k}=z$ on $Z$ and $z_{k}=1 / k$ on $\Gamma \backslash Z$. Then $z_{k} \rightarrow z$ uniformly on $\Gamma$. But for all $k \in \mathbb{N}$ we have $\operatorname{supp} z_{k}=\Gamma \nrightarrow \operatorname{supp} z=Z$ and hence, $\inf (\rho)=\rho(k)=\operatorname{dist}(\operatorname{supp} z, \partial \Gamma)$ for all $k \in \mathbb{N}, c f$. (6.2). Thus, $\operatorname{supp} z_{k} \subset \operatorname{supp} z+B_{\rho(k)}(0)$, but $\rho(k) \not 0$.

To exclude situations as above it is essential that $z_{k}(x) \in\{0,1\}$ a.e. on $\Gamma$, which is indeed given by the set $\operatorname{SBV}(\Gamma ;\{0,1\})$. Hence, $z_{k}$ is the characteristic function of the finite-perimeter set $Z_{k}$ as in (6.9).

However, working in $\operatorname{SBV}(\Gamma ;\{0,1\})$ in general neither ensures

$$
Z_{k}=\operatorname{supp} z_{k} \quad \mathcal{H}^{m} \text {-a.e. on } \Gamma,
$$

nor support convergence (6.3). This can be seen from Example 2 below, which is constructed in the spirit of ([33], p. 24, Rem. 1.27) or ([4], p. 154, example 3.53). In fact, (6.11) and (6.3) will be deduced only by exploiting an additional qualification, namely the semistability (6.5).

Example 2. Let $Q:=(0,1)^{2}$. The set of points with rational coordinates $Q \cap \mathbb{Q}^{2}$ is countable and can be arranged in a sequence $\left(q_{j}\right)_{j}$. For every $j \in \mathbb{N}$ and every $k \in \mathbb{N}$ we define the open ball $B\left(q_{j}, r_{j k}\right)$ with radius $r_{j k}:=1 /\left(4 k \cdot 2^{j}\right)$ and center in $q_{j}$. Then, $\mathcal{L}^{2}\left(B\left(q_{j}, r_{j k}\right)\right)=\pi /\left(16 k^{2} \cdot 2^{2 j}\right)$ and $P\left(B\left(q_{j}, r_{j k}\right), Q\right)=\pi /\left(2 k \cdot 2^{j}\right)$. For all $k \in \mathbb{N}$ we set $Z_{k}:=\cup_{j \in \mathbb{N}} B\left(q_{j}, r_{j k}\right)$ and as $k \rightarrow \infty$ we obtain that

$$
\mathcal{L}^{2}\left(Z_{k}\right) \leq \sum_{j=1}^{\infty} \mathcal{L}^{2}\left(B\left(q_{j}, r_{j k}\right)\right)=\pi /\left(8 k^{2}\right) \rightarrow 0, \quad P\left(Z_{k}, Q\right) \leq \sum_{j=1}^{\infty} P\left(B\left(q_{j}, r_{j k}\right), \Gamma\right)=\pi / k \rightarrow 0 .
$$

Hence $z_{k} \rightarrow z$ in $L^{1}(Q)$, where $Z=\emptyset$ (which can be identified with $Q \cap \mathbb{Q}^{2}$, in the sense that the respective indicator functions differ on a set of null Lebesgue measure). The perimeters as well converge, since $P\left(Z_{k}, Q\right) \rightarrow$ $0=P\left(Q \cap \mathbb{Q}^{2}, Q\right)$. Notice that, since $Q \cap \mathbb{Q}^{2} \subset Z_{k} \subset Q$ the sets $Z_{k}$ are dense in $Q$ for all $k \in \mathbb{N}$. Hence, by formula (6.8) we have $\operatorname{supp} z_{k}=Q$ for all $k \in \mathbb{N}$, whereas $\operatorname{supp} z=\emptyset$. This discrepancy is due to the fact that $\left(Z_{k}\right)_{k}$ converge to a dense set of zero $\mathcal{L}^{2}$-measure, while $\mathcal{L}^{2}\left(Z_{k}\right)<\mathcal{L}^{2}\left(\operatorname{supp} z_{k}\right)$ because the topological boundary $\partial Z_{k}$ of the sets $Z_{k}$ is of positive $\mathcal{L}^{2}$-measure. Thus, $\operatorname{supp} z_{k} \nrightarrow \operatorname{supp} z$. In particular, support convergence (6.3) does not hold, because $\emptyset+B_{\rho(k)}(0)=\emptyset$ and hence $\operatorname{supp} z_{k} \not \subset \emptyset$ for any $\rho(k)>0$.

Examples 1 and 2 suggest that there are two reasons for the failure of support convergence (6.3) under convergence (6.1):

$$
\begin{aligned}
& \text { 1. } \operatorname{supp} z=\emptyset \text { and } \operatorname{supp} z_{k} \neq \emptyset \text { for all } k \in \mathbb{N} \text {. } \\
& \text { 2. } \operatorname{supp} z \neq \emptyset \text { and } \quad \rho(k) \not \rightarrow 0 \text { as } k \rightarrow \infty .
\end{aligned}
$$

Now, in the ensuing Propositions 6.7 and 6.8 we will exclude (6.12a) and (6.12b), respectively, for semistable sequences $\left(z_{k}\right)_{k}$. Let us now roughly outline our argument. Because of $z_{k} \rightarrow z$ in $L^{1}(\Gamma)$ by (6.1), we observe that $z_{k} \rightarrow 0$ in $L^{1}\left(N_{z}\right)$. For the associated finite-perimeter sets $Z_{k}$ as in (6.9) we have

$$
\mathcal{H}^{m}\left(Z_{k} \cap N_{z}\right) \rightarrow 0 \quad \text { as } k \rightarrow \infty .
$$




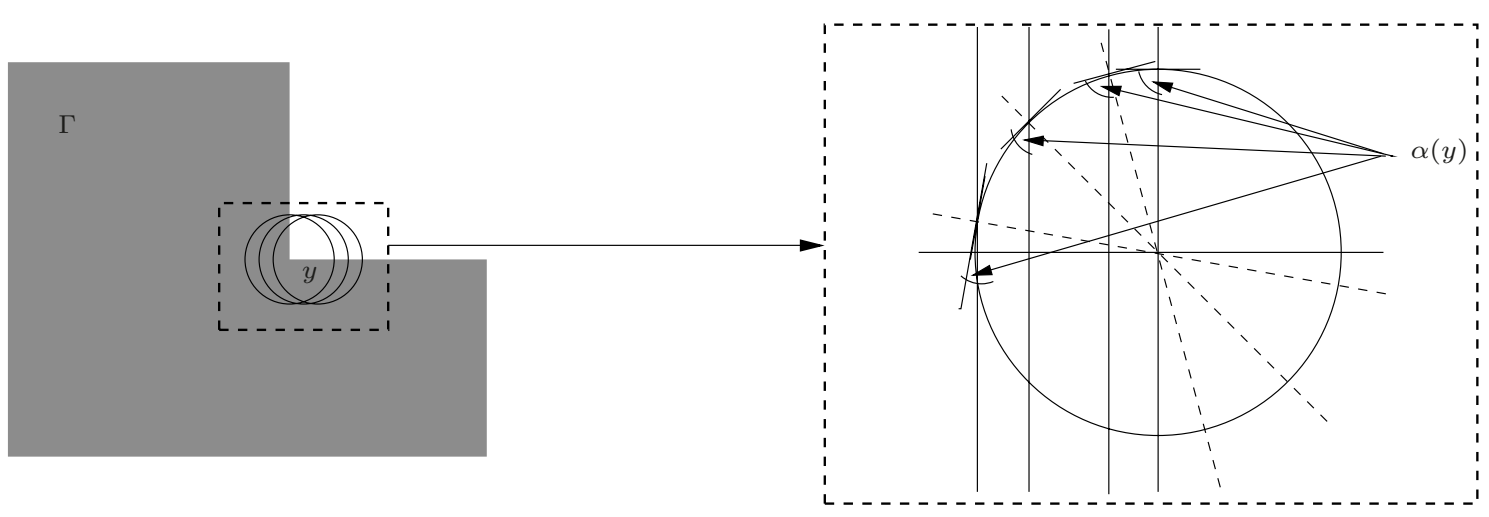

FiguRE 2. The intersection angle $\alpha(y) \rightarrow 0$ as $y$ moves from the reentrant corner to the right.

In other words, $Z_{k} \cap N_{z}$ turns into a set with arbitrarily small $\mathcal{H}^{m}$-measure. On the other hand, from (6.12a) and $(6.12 \mathrm{~b})$ we will deduce that there exist points $y_{k} \in \operatorname{supp} z_{k} \cap N_{z}$ and a fixed radius $\rho_{\star}<R$ such that $B_{\rho_{\star}}\left(y_{k}\right) \cap \Gamma \subset N_{z}$. Hence, $Z_{k} \cap B_{\rho_{\star}}\left(y_{k}\right) \subset Z_{k} \cap N_{z}$. Now, taking into account the lower density estimate (6.6), we will then arrive at a contradiction to (6.13). Hence, we will rule out (6.12a) and (6.12b).

Outline of the proof of Theorem 6.1. As the key ingredient we will prove the lower density estimate (6.6), for semistable sets in Theorem 6.6. The proof of Theorem 6.1 will be completed by deducing support convergence for the case $\operatorname{supp} z=\emptyset$ in Proposition 6.7 and for the case $\operatorname{supp} z \neq \emptyset$ in Proposition 6.8, by exploiting Theorem 6.6.

The main tool to prove (6.6) is a relative isoperimetric inequality in $\Gamma \cap B_{\rho}(y)$, i.e. in $\Gamma$ intersected with a ball $B_{\rho}(y)$ with center in $y \in \bar{\Gamma}$ and radius $\rho>0$. The isoperimetric constant $C_{\Gamma}$ solely depends on the cone defining the cone property of the convex domain $\Gamma \subset \mathbb{R}^{m}$ and on the space dimension $m$. In particular, $C_{\Gamma}$ is independent of the choice of $y \in \bar{\Gamma}$ and $\rho>0$. Thus, setting $\rho>0$ sufficiently large such that $\Gamma \cap B_{\rho}(y)=\Gamma$ yields a relative isoperimetric inequality in $\Gamma$.

Theorem 6.3 (Uniform relative isoperimetric inequality [63], Thm. 3.2).

Let $m>1$ be an integer and $\Gamma \subset \mathbb{R}^{m}$ a convex domain. Let $A \subset \Gamma$ be a set of finite perimeter in $\Gamma$. There exists a constant $C_{\Gamma}$, such that for all $y \in \bar{\Gamma}$ and for all $\rho>0$ it is

$$
\min \left\{\mathcal{H}^{m}\left(A \cap\left(\Gamma \cap B_{\rho}(y)\right)\right), \mathcal{H}^{m}\left(\left(\Gamma \cap B_{\rho}(y)\right) \backslash A\right)\right\}^{\frac{m-1}{m}} \leq C_{\Gamma} P\left(A,\left(\Gamma \cap B_{\rho}(y)\right)\right),
$$

where the constant $C_{\Gamma}$ solely depends on the cone defining the cone property of the convex domain $\Gamma \subset \mathbb{R}^{m}$, on its diameter and on the space dimension $m$.

Remark 6.4 (On the uniform relative isoperimetric inequality).

The assumption of convexity on the Lipschitz-domain $\Gamma$ is essential for the proof of the uniform relative isoperimetric inequality in Theorem 6.3 for the following reasons: The proof exploits that every domain $\Gamma \cap B_{\rho}(y)$ for $y \in \bar{\Gamma}$ satisfies the cone property with a cone of the same opening angle as the one of $\Gamma$. This is due to the fact that the intersection angle $\alpha(y)$ of the boundary $\partial \Gamma$ and a ball $B_{\rho}(y)$ with center $y \in \bar{\Gamma}$ is at least $90^{\circ}$ for a convex domain $\Gamma$. Hence, the cone defining the cone property for $\Gamma \cap B_{\rho}(y)$ may have a smaller height than the one for $\Gamma$, but the opening angles of the cones are the same. In this case the cones can be scaled to the same size by a suitable scaling of $\Gamma \cap B_{\rho}(y)$. In contrast, for a non-convex domain $\Gamma$, the intersection angle $\alpha(y)$ can degenerate to zero as the center $y$ moves along the boundary $\partial \Gamma$ away from a reentrant corner, see Figure 2. Therefore, the opening angle of the cone differs for every domain $\Gamma \cap B_{\rho}(y)$ in dependence of the location of $y \in \bar{\Gamma}$. Thus, in the non-convex case, the cones of $\Gamma$ and $\Gamma \cap B_{\rho}(y)$ cannot be transformed into each other simply by scaling. 
Proof of the lower density estimate (6.6) for semistable sets. In the following we deduce (6.6) for $z_{k}$ being semistable w.r.t. $\Phi_{k}\left(u_{k}, \cdot\right)$, hence w.r.t. $\mathcal{S}(\cdot)(c f .(6.5))$, for every $k \in \mathbb{N} \cup\{\infty\}$ fixed. The proof of (6.6) is developed by contradiction, i.e. instead of (6.6) we have

$$
\begin{array}{ll}
\exists y \in \operatorname{supp} z_{k} \exists \rho_{\star} \in(0, R): & \mathcal{H}^{m}\left(Z_{k} \cap B_{\rho_{\star}}(y)\right)<\mathfrak{a}(\Gamma) \rho_{\star}^{m}, \\
\exists y \in \operatorname{supp} z_{k} \quad \exists \rho_{\star}>R: & \mathcal{H}^{m}\left(Z_{k} \cap B_{\rho_{\star}}(y)\right)<\mathfrak{a}(\Gamma) R^{m},
\end{array}
$$

where the constants $R$ and $\mathfrak{a}(\Gamma)$ will be determined in what follows ( $c f .(6.23))$. In the following lines, we will drop the index $k$. In particular, our aim is to show that, assuming the validity of (6.15), the semistability (6.5) of a set $Z$ for $\mathcal{S}$ (resp. its characteristic function $z$ ), is violated for a particular test function $\tilde{z}$ being the characteristic function of a suitable set $\widetilde{Z}$. Since we are working in $\operatorname{SBV}(\Gamma ;\{0,1\})$ this test function $\tilde{z}$ can be constructed by "cutting off" a suitable subset of $Z \cap B_{\rho_{\star}}(y)$. More detailed, this cut-off will yield that $\widetilde{Z}^{1} \subset Z^{1}$. Additionally, it may generate a new surface and we have to ensure that this new surface is smaller than the surface of the part which is cut off. To show the existence of a suitable cut-off for $Z \cap B_{\rho_{\star}}(y)$ of sufficiently small $\mathcal{H}^{m}$-measure we are going to check that for a general set $A \subset \Gamma$ with $P(A, \Gamma)<\infty$ :

$$
\begin{aligned}
& 0<\mathcal{H}^{m}\left(A \cap B_{\rho_{\star}}(y)\right)<M_{\rho_{\star}} \Rightarrow \exists \rho \in\left[\rho_{\star} / 2, \rho_{\star}\right]: 0<\mathcal{H}^{m-1}\left(A \cap \partial B_{\rho}(y)\right)<\frac{1}{2} P\left(A, \Gamma \cap B_{\rho}(y)\right), \\
& \text { where } M_{\rho_{\star}}:=\min \left\{I\left(\Gamma, \rho_{\star}, y\right) / 2, I\left(\Gamma, \rho_{\star}, y\right) /\left(\omega_{m}\left(2 C_{\Gamma} m\right)^{m}\right), \mathrm{b}^{m} /\left(2 C_{\Gamma}\left(a_{0}+a_{1}\right)\right)^{m}\right\} \\
& \text { with } I\left(\Gamma, \rho_{\star}, y\right):=\mathcal{H}^{m}\left(\Gamma \cap B_{\rho_{\star} / 2}(y)\right) \text { and } \omega_{m}:=\mathcal{H}^{m}\left(B_{1}(0)\right),
\end{aligned}
$$

where $\rho_{\star}$ is from (6.15). In the proof of the lower density estimate ( $c f$. Thm. 6.6 below), we will then test the semistability of $Z$, in particular (6.5), with the characteristic function $\tilde{z}$ of the set $\widetilde{Z}:=Z \backslash B_{\rho}(y)$, with $\rho \in\left[\rho_{\star} / 2, \rho_{\star}\right]$ such that estimate (6.16a) holds, and arrive to a contradiction with the semistability. Hence we will deduce that (6.15) cannot hold true, whence the desired (6.6).

In order to verify implication (6.16a) we assume the contrary, i.e.

$$
\mathcal{H}^{m}\left(A \cap B_{\rho_{\star}}(y)\right)<M_{\rho_{\star}} \text { and } \forall \rho \in\left[\rho_{\star} / 2, \rho_{\star}\right]: \mathcal{H}^{m-1}\left(A \cap \partial B_{\rho}(y)\right) \geq \frac{1}{2} P\left(A, \Gamma \cap B_{\rho}(y)\right) .
$$

For the contradiction argument we will use the volume formula (cf. [17], Chap. 3.4.4, Prop. 1, p. 118)

$$
\forall R>0: y \in A \subset \mathbb{R}^{m}, A \subset B_{R}(y) \quad \Rightarrow \quad \mathcal{H}^{m}(A)=\int_{0}^{R} \mathcal{H}^{m-1}\left(A \cap \partial B_{\rho}(y)\right) \mathrm{d} \rho
$$

and we will exploit the uniform relative isoperimetric inequality in $\Gamma$ intersected with balls, $c f$. (6.14).

Lemma 6.5. Assume (3.7c). Let $A$ be a set with finite perimeter in $\Gamma$. Then implication (6.16a) is true.

Proof. We assume that (6.16a) is false, i.e. we have (6.17), instead. By (6.16b) it is $\mathcal{H}^{m}\left(A \cap B_{\rho_{\star}}(y)\right)<M_{\rho_{\star}} \leq$ $\mathcal{H}^{m}\left(\Gamma \cap B_{\rho_{\star} / 2}(y)\right) / 2$ and hence, as $A \subset \Gamma$, we have

$$
\forall \rho \in\left[\rho_{\star} / 2, \rho_{\star}\right]: \min \left\{\mathcal{H}^{m}\left(A \cap\left(\Gamma \cap B_{\rho}(y)\right)\right), \mathcal{H}^{m}\left(\left(\Gamma \cap B_{\rho}(y)\right) \backslash A\right)\right\}=\mathcal{H}^{m}\left(A \cap B_{\rho}(y)\right) .
$$

Moreover, applying the relative isoperimetric inequality (6.14) on the estimate in (6.17) yields that for all $\rho \in\left[\rho_{\star} / 2, \rho_{\star}\right]$ it is

$$
\mathcal{H}^{m-1}\left(A \cap \partial B_{\rho}(y)\right) \geq \frac{1}{2 C_{\Gamma}} \mathcal{H}^{m}\left(A \cap B_{\rho}(y)\right)^{\frac{m-1}{m}},
$$

where $\mathcal{H}^{m-1}\left(A \cap \partial B_{\rho}(y)\right)=\frac{\mathrm{d}}{\mathrm{d} \rho} \mathcal{H}^{m}\left(A \cap B_{\rho}(y)\right)$ by (6.18). Since $\mathcal{H}^{m}\left(A \cap B_{\rho}(y)\right)>0$ for all $\rho \in\left[\rho_{\star} / 2, \rho_{\star}\right]$, we can divide by $\mathcal{H}^{m}\left(A \cap B_{\rho}(y)\right)^{\frac{m-1}{m}}$ in (6.20). Integration over $\rho \in\left(\rho_{\star} / 2, \rho_{\star}\right)$ with the substitution $u=\mathcal{H}^{m}\left(A \cap B_{\rho}(y)\right)$, 
$\mathrm{d} u=\mathcal{H}^{m-1}\left(A \cap \partial B_{\rho}(y)\right) \mathrm{d} \rho$ then yields

$$
\begin{aligned}
I: & =\int_{\rho_{\star} / 2}^{\rho_{\star}} \mathcal{H}^{m}\left(A \cap B_{\rho}(y)\right)^{\frac{1-m}{m}} \mathcal{H}^{m-1}\left(A \cap \partial B_{\rho}(y)\right) \mathrm{d} \rho=\int_{a}^{b} u^{\frac{1-m}{m}} \mathrm{~d} u=\left[m u^{\frac{1}{m}}\right]_{a}^{b} \\
& =m\left(\mathcal{H}^{m}\left(A \cap B_{\rho_{\star}}(y)\right)^{\frac{1}{m}}-\mathcal{H}^{m}\left(A \cap B_{\rho_{\star} / 2}(y)\right)^{\frac{1}{m}}\right),
\end{aligned}
$$

where we have used $a=\mathcal{H}^{m}\left(A \cap B_{\rho_{\star} / 2}(y)\right)$ and $b=\mathcal{H}^{m}\left(A \cap B_{\rho_{\star}}(y)\right)$ for shorter notation. Altogether, (6.20) then implies $I \geq\left(2 C_{\Gamma}\right)^{-1} \int_{\rho_{\star} / 2}^{\rho_{\star}} \mathrm{d} \rho=\rho_{\star} /\left(4 C_{\Gamma}\right)$. This leads to the contradiction

$$
0<\frac{\rho_{\star}}{4 C_{\Gamma} m} \leq\left(\mathcal{H}^{m}\left(A \cap B_{\rho_{\star}}(y)\right)^{\frac{1}{m}}-\mathcal{H}^{m}\left(A \cap B_{\rho_{\star} / 2}(y)\right)^{\frac{1}{m}}\right)<\left(\frac{I\left(\Gamma, \rho_{\star}, y\right)}{\omega_{m}\left(2 C_{\Gamma} m\right)^{m}}\right)^{1 / m} \leq \frac{\rho_{\star}}{4 C_{\Gamma} m},
$$

where we neglected $-\mathcal{H}^{m}\left(A \cap B_{\rho_{\star} / 2}(y)\right)^{\frac{1}{m}}<0$ and exploited that $\mathcal{H}^{m}\left(A \cap B_{\rho_{\star}}(y)\right)<\frac{I\left(\Gamma, \rho_{\star}, y\right)}{\omega_{m}\left(2 C_{\Gamma} m\right)^{m}}$ by $(6.16 \mathrm{~b})$ and the prerequisite in (6.16a). The last inequality in the above chain is due to the very Definition (6.16c) of $I\left(\Gamma, \rho_{\star}, y\right)$. We conclude that $(6.17)$ is false, thus (6.16a) holds true.

In Theorem 6.6 below we derive (6.6) by leading its converse, i.e. (6.15), to a contradiction to the semistability of $Z$, with the aid of implication (6.16a). For this, we choose $\mathfrak{a}(\Gamma)$ and $R$ such that $\mathcal{H}^{m}\left(Z \cap B_{\rho_{\star}}(y)\right)<\mathfrak{a}(\Gamma) R^{m}$ implies $\mathcal{H}^{m}\left(Z \cap B_{\rho_{\star}}(y)\right)<M_{\rho_{\star}}$ : in fact, we estimate the constant $M_{\rho_{\star}}$, cf. formulae (6.16b) and (6.16c), from below in terms of $\mathfrak{a}(\Gamma) \rho_{\star}^{m}$. To do so, we exploit that the Lipschitz domain $\Gamma$ satisfies the uniform cone property with a cone $C(\theta, h, \xi)$ of opening angle $\theta$, height $h$ and axis $\xi$. In particular, there is a radius $r>0$ such that

$$
\forall x \in \partial \Gamma \forall y \in B_{r}(x): \quad y+C\left(\theta, h, \xi_{x}\right) \subset \Gamma .
$$

Since, for all $\rho_{\star}>0$, a cone $C\left(\theta, \rho_{\star}, \xi\right)$ of height $\rho_{\star}$ is proportional to the ball of radius $\rho_{\star}$ by a constant $k(\theta)>0$, we find

$$
\forall x \in \partial \Gamma \forall \rho_{\star} \in(0,2 h): \mathcal{H}^{m}\left(\Gamma \cap B_{\rho_{\star} / 2}(x)\right) \geq \mathcal{H}^{m}\left(C\left(\theta, \rho_{\star} / 2, \xi_{x}\right)\right)=2^{-m} k(\theta) \omega_{m} \rho_{\star}^{m} .
$$

In view of $(6.16 \mathrm{~b})$ and $(6.22)$ we choose

$$
\mathfrak{a}(\Gamma):=2^{-m} k(\theta) \omega_{m} / \max \left\{2, \omega_{m}\left(2 C_{\Gamma} m\right)^{m},\left(2 C_{\Gamma}\left(a_{0}+a_{1}\right)\right)^{m}\right\} \text { and } R \leq \min \left\{2 h, 2^{m} \omega_{m}^{-1 / m} \mathrm{~b}\right\} .
$$

Hence, (6.23) now allows us to verify (6.6).

Theorem 6.6 (Lower density estimate for semistable elements).

Assume (3.7c). Let $k \in \mathbb{N} \cup\{\infty\}$ and $z_{k}$ the characteristic function of the finite perimeter set $Z_{k}$, semistable for $\Phi\left(u_{k}, \cdot\right)$, hence for $\mathcal{S}(\cdot)$. Then the lower density estimate $(6.6)$ holds with the constants $\mathfrak{a}(\Gamma)$ and $R$ given by $(6.23)$.

Proof. For simpler notation we again drop the index $k$ within this proof. Let $Z$ be a set of finite perimeter and $z$ its characteristic function, semistable for $\mathcal{S}(\cdot)$, i.e. (6.5) holds true. Let $y \in \operatorname{supp} z$ be given by (6.15) with the respective constants $R>0$ and $\mathfrak{a}(\Gamma)$ determined by (6.23). Hence in both cases of (6.15) we have $\mathcal{H}^{m}\left(Z \cap B_{\rho_{\star}}(y)\right)<M_{\rho_{\star}}$ with $M_{\rho_{\star}}$ from (6.16b) and thus, implication (6.16a) is valid.

We test the semistability of $Z$, in particular (6.5), with the characteristic function $\tilde{z}$ of the set $\widetilde{Z}:=Z \backslash B_{\rho}(y)$, with $\rho \in\left[\rho_{\star} / 2, \rho_{\star}\right]$ such that estimate $(6.16 \mathrm{a})$ holds. In particular, the above construction of $\tilde{z}$ ensures that $\tilde{z} \leq z$, so that $\mathcal{R}_{1}(\tilde{z}-z)=\int_{\Gamma} a_{1}(z-\tilde{z}) \mathrm{d} \mathcal{H}^{d-1}$. Moreover, in view of Definitions A.10, A.15 and (A.45), it yields $\mathrm{J}_{\tilde{z}}=\mathfrak{F} Z \backslash\left(\mathfrak{F} Z \cap B_{\rho}(y)\right) \cup\left(Z \cap \partial B_{\rho}(y)\right)$. Hence, (6.5) leads to the following relation

$$
\mathrm{b} P\left(Z, \Gamma \cap B_{\rho}(y)\right) \leq\left(a_{0}+a_{1}\right) \mathcal{H}^{m}\left(Z \cap B_{\rho}(y)\right)+\mathrm{b} \mathcal{H}^{m-1}\left(Z \cap \partial B_{\rho}(y)\right) .
$$


Property (6.16a) implies that $P\left(Z, \Gamma \cap B_{\rho}(y)\right)>0$. Hence, (6.24) is equivalent to

$$
\mathrm{b} \leq\left(a_{0}+a_{1}\right) \frac{\mathcal{H}^{m}\left(Z \cap B_{\rho}(y)\right)}{P\left(Z, \Gamma \cap B_{\rho}(y)\right)}+\mathrm{b} \frac{\mathcal{H}^{m-1}\left(Z \cap \partial B_{\rho}(y)\right)}{P\left(Z, \Gamma \cap B_{\rho}(y)\right)}
$$

where $\mathcal{H}^{m-1}\left(Z \cap \partial B_{\rho}(y)\right) / P\left(Z, \Gamma \cap B_{\rho}(y)\right)<\frac{1}{2}$ by (6.16a). Also note that the relative isoperimetric inequality (6.14) ensures

$$
0<\mathcal{H}^{m}\left(Z \cap B_{\rho}(y)\right)=\min \left\{\mathcal{H}^{m}\left(Z \cap\left(\Gamma \cap B_{\rho}(y)\right)\right), \mathcal{H}^{m}\left(\left(B_{\rho}(y) \cap \Gamma\right) \backslash Z\right)\right\} .
$$

Hence, one more application of (6.14) in (6.25), together with (6.26) and (6.16b) yields

$$
\left(a_{0}+a_{1}\right) \frac{\mathcal{H}^{m}\left(Z \cap B_{\rho}(y)\right)}{P\left(Z, \Gamma \cap B_{\rho}(y)\right)} \leq\left(a_{0}+a_{1}\right) C_{\Gamma} \mathcal{H}^{m}\left(Z \cap B_{\rho}(y)\right)^{\frac{1}{m}}<\mathrm{b} / 2 .
$$

Inserting these estimates into (6.25) then generates the contradiction $1<1$, which concludes the proof.

Proof of Theorem 6.1. With the aid of Theorem 6.6 we are now in a position to verify the support convergence. In the case of $\operatorname{supp} z=\emptyset$ we will apply estimate $(6.6 \mathrm{~b})$ with $\rho_{\star}:=\operatorname{diam} \Gamma$ (the diameter of $\Gamma$ ), while the case $\operatorname{supp} z \neq \emptyset$ will follow from estimate (6.6a). We start with the case $\operatorname{supp} z=\emptyset$ and show that (6.12a) is excluded.

Proposition 6.7 (Support convergence of semistable sequences if $\operatorname{supp} z=\emptyset$ ).

Assume (3.7c). Let $\left(z_{k}\right)_{k}, z \subset L^{\infty}(0, T ; \operatorname{SBV}(\Gamma ;\{0,1\}))$ be as in Theorem 6.1. Assume that $\operatorname{supp} z(t)=\emptyset$ at some $t \in(0, T)$. Then, there is an index $k_{0}(t) \in \mathbb{N}$ such that also $\operatorname{supp} z_{k}(t)=\emptyset$ for all $k \geq k_{0}(t)$.

Proof. Since $\operatorname{supp} z(t)=\emptyset$ and $z_{k}(t) \stackrel{*}{\rightarrow} z(t)$ in $\operatorname{SBV}(\Gamma ;\{0,1\})$, we have $z_{k}(t) \rightarrow 0$ in $L^{1}(\Gamma)$. For every $k \in \mathbb{N}$ we choose a point $y_{k} \in \operatorname{supp} z_{k}$. Moreover, we fix $\rho_{\star}:=\operatorname{diam} \Gamma$. This choice ensures that $\Gamma \subset B_{\rho_{\star}}\left(y_{k}\right)$ for all $k \in \mathbb{N}$. Hence, $z_{k}(t) \rightarrow 0$ in $L^{1}(\Gamma)$ is equivalent to $\mathcal{H}^{m}\left(Z_{k} \cap B_{\rho_{\star}}\left(y_{k}\right)\right) \rightarrow 0$. Thus, there is an index $k_{0} \in \mathbb{N}$ such that the lower density estimate $(6.6 \mathrm{~b})$ is violated for all $k \geq k_{0}$, which is in contradiction to the semistability of $z_{k}$ for $\Phi_{k}$. Therefore, we conclude that $z_{k} \equiv 0$ for all $k \geq k_{0}$.

Proposition 6.8 (Support convergence for semistable sequences if $\operatorname{supp} z \neq \emptyset$ ).

Assume (3.7c) and $\operatorname{supp} z \neq \emptyset$. Let $\left(z_{k}\right), z \subset L^{\infty}(0, T ; \operatorname{SBV}(\Gamma ;\{0,1\}))$ be as in Theorem 6.1. Then the support convergence (6.3) holds true.

Proof. Fix $t \in(0, T)$ outside a set of null Lebesgue measure, such that convergence (6.1) and semistability (6.5) hold for $\left(z_{k}(t)\right)_{k}$. For shorter notation we omit to indicate the time-dependence of $z_{k}$ and $\rho(k)$. Let us proceed by contradiction and assume that $(6.3)$ does not hold. The sequence $(\rho(k))_{k}$ is uniformly bounded by the boundedness of $\Gamma$. Hence, we can find a subsequence converging to the limsup of the whole sequence $\lim \sup _{k \rightarrow \infty} \rho(k)=: \rho_{0}$. Moreover, due to $z_{k} \rightarrow z$ in $L^{1}(\Gamma)$, there is a further subsequence which converges pointwise a.e. on $\Gamma$. For this subsequence the contradiction to (6.3) reads

$$
\operatorname{supp} z_{k} \subset \operatorname{supp} z+B_{\rho(k)}(0) \text { for all } k \in \mathbb{N} \text { and } \rho(k) \rightarrow \rho_{0}>0 \text { as } k \rightarrow \infty .
$$

Because of $\rho_{0}>0$ we can find a further subsequence and an index $k_{0}$ such that

$$
\rho(k)>\rho_{0} / 2 \text { for all } k \geq k_{0} .
$$

Assume that $\mathcal{H}^{m}\left(\operatorname{supp} z_{k} \cap N_{z}\right)>0$ (otherwise $\rho(k)=0$ ). Then, also $\mathcal{H}^{m}\left(Z_{k} \cap N_{z}\right)>0$. Thus, in view of (6.10) for every $k \geq k_{0}$ there is a point $y_{k} \in \operatorname{supp} z_{k} \cap N_{z}$ with the property

$$
\operatorname{dist}\left(y_{k}, \operatorname{supp} z\right) \geq \rho_{0} / 2 \text { and thus } B_{\rho_{0} / 4}\left(y_{k}\right) \cap \Gamma \subset N_{z} .
$$

Hereby, $\rho_{0} / 4>0$ satisfies either $\rho_{0} / 4 \in(0, R)$ or $\rho_{0} / 4>R$ for $R$ as in (6.23). Correspondingly, since the functions $z_{k}$ are semistable, the sets $Z_{k} \cap B_{\rho_{0} / 4}\left(y_{k}\right)$ satisfy the lower density estimate with either (6.6a) or (6.6b). 
However, the convergence $z_{k} \rightarrow z$ pointwise $\mathcal{H}^{m}$-a.e. implies that for every $\varepsilon \in(0,1)$ and $\mathcal{H}^{m}$-a.a. $y \in N_{z}$ there is an index $k(y, \varepsilon)$ such that for all $k \geq k(y, \varepsilon)$ it holds $\left|z_{k}(y)\right|=\left|z_{k}(y)-z(y)\right|<\varepsilon$, hence

$$
z_{k} \stackrel{*}{\rightarrow} z \text { in } \operatorname{SBV}(\Gamma ;\{0,1\}) \Rightarrow \mathcal{H}^{m}\left(Z_{k} \cap B_{\rho_{0} / 4}\left(y_{k}\right)\right) \leq \mathcal{H}^{m}\left(Z_{k} \cap N_{z}\right) \rightarrow 0 \text { as } k_{0} \leq k \rightarrow \infty
$$

for the finite perimeter sets $Z_{k}$ underlying the characteristic functions $z_{k}$. Therefore, necessarily, there is an index $k_{\star}>k_{0}$ such that for all $k \geq k_{\star}$ the lower density estimate (6.6) is violated, which is in contradiction to the semistability of $z_{k}$. Therefore, $\rho_{0}>0$ does not hold. This implies that $\rho(k) \rightarrow 0$ for the whole sequence $(\rho(k))_{k}$ and hence support convergence holds for the whole sequence $\left(z_{k}\right)_{k}$.

Combining the results on support convergence with the strong $L^{1}$-convergence of semistable sequences finally allows us to conclude the Hausdorff convergence of the respective supports.

Corollary 6.9 (Hausdorff convergence of the supports).

Let the assumptions of Theorem 6.1 hold true. Then, for all $t \in[0, T]$ the sequence of closed sets $\left(\operatorname{supp} z_{k}(t)\right)_{k}$ Hausdorff converges to $\operatorname{supp} z(t)$.

Proof. Again, within this proof we omit to indicate the variable $t$. Since support convergence (6.3) holds true by Propositions 6.7 and 6.8, it remains to verify convergence (6.4). For this, note that $\operatorname{supp} z_{k}=\left(\operatorname{supp} z_{k} \cap\right.$ $\operatorname{supp} z) \cup\left(\operatorname{supp} z_{k} \backslash \operatorname{supp} z\right)$ and for the latter term we have convergence according to (6.3). Thus, only the first term is relevant for (6.4) and thereto we may w.l.o.g. consider the case $\operatorname{supp} z_{k} \subset \operatorname{supp} z$ for all $k \in \mathbb{N}$.

Let $x \in \partial \operatorname{supp} z$ be arbitrary but fixed. Hence, for all $\varepsilon>0$ we have $\mathcal{H}^{m}\left(Z \cap B_{\varepsilon}(x)\right)>0$. Moreover, due to $z_{k} \rightarrow z$ in $L^{1}(\Gamma)$ there holds $\mathcal{H}^{m}\left(\left(\left(Z \backslash Z_{k}\right) \cup\left(Z_{k} \backslash Z\right)\right) \cap B_{\varepsilon}(x)\right) \rightarrow 0$. Thus, there is an index $k(x, \varepsilon)$ such that, for all $k \geq k(x, \varepsilon)$ it is $\mathcal{H}^{m}\left(Z_{k} \cap B_{\varepsilon}(x)\right)>0$. But this implies $\operatorname{dist}\left(\partial \operatorname{supp} z_{k}, \partial \operatorname{supp} z\right) \rightarrow 0$ as $k \rightarrow \infty$ and hence (6.4).

Remark 6.10. We note that Propositions 6.7 and 6.8 only require the semistability of the delamination variables $\left(z_{k}\right)_{k}$ of the SBV-adhesive contact systems: The semistability of the limit function $z$ is not needed. Nonetheless, the proof of the semistability for the limit function is completely independent from the support convergence property (6.3).

Remark 6.11 (Generality).

The results of Theorem 6.6 and Propositions 6.7 and 6.8 are solely based on semistability and strong $L^{1}$-convergence. In other words, further properties of the delamination models such as temperature dependence or visco-elasticity have no influence. In particular, Propositions 6.7 and 6.8 also hold for energetic solutions in the fully rate-independent setting, where solutions are characterized as satisfying an energy balance and a global stability condition, see (1.6).

Remark 6.12 (Open problem: from brittle SBV-delamination to Griffith-type delamination).

It remains an open problem if it is possible to get rid of the SBV-gradient regularization, like in [49] in the limit passage from Sobolev gradient to Griffith-type delamination. In the present context, this would mean passing to zero with the coefficient $\mathrm{b}$ in the gradient term $\mathcal{G}_{\mathrm{b}}(z):=\mathrm{b} P(Z, \Gamma)$ contributing to the energy $\Phi_{\mathrm{b}}$.

Seemingly, the main difficulty attached to the limit passage as $\mathrm{b} \rightarrow 0$ is the proof of the support convergence (5.3), which in turn would be crucial for passing to the limit in the momentum equation in this case as well. More specifically, we highlight that Theorem 6.6 excludes the presence of subsets $Z_{k} \cap B_{\rho_{\star}}\left(y_{k}\right)$ with $\mathcal{H}^{m}\left(Z_{k} \cap B_{\rho_{\star}}\left(y_{k}\right)\right)<M_{\rho_{\star}}$. The bound $M_{\rho_{\star}}$ on the measure of $Z_{k} \cap B_{\rho_{\star}}\left(y_{k}\right)$ explicitly involves the constant $\mathrm{b}>0$, see $(6.16 \mathrm{~b})$. In fact, the passage from SBV-brittle delamination to Griffith-type delamination as $\mathrm{b} \rightarrow 0$ would bring along a loss of uniform boundedness in $\operatorname{SBV}(\Gamma)$ for the sequence $\left(z_{\mathrm{b}}\right)_{\mathrm{b}}$ of delamination variables for the SBV-brittle delamination systems. Indeed, only the uniform bound in $L^{\infty}(\Gamma)$ would remain. Hence, the limit of a semistable sequence $\left(z_{\mathrm{b}}\right)_{\mathrm{b}} \subset \operatorname{SBV}(\Gamma ;\{0,1\})$ with $z_{\mathrm{b}} \stackrel{*}{\rightarrow} z$ in $L^{\infty}(\Gamma)$ would be an $L^{\infty}$-function, only, which can of course contain concentrating subsets. Indeed, for $\mathrm{b} \rightarrow 0$, the uniform lower bound on the measure of subsets $Z_{k} \cap B_{\rho_{*}}\left(y_{k}\right)$ is lost and the larger the perimeters of the approximating functions may get, the smaller the subsets preventing support convergence may become. 


\section{A DifFERENT SCALING FOR THE PASSAGE FROM ADHESIVE TO BRITTLE}

In what follows we briefly discuss an alternative scaling, in the mechanical energy and in the dissipation potential, for taking the brittle limit as $k \rightarrow \infty$ in the SBV-adhesive contact system. Namely, we scale the dissipation density $\mathrm{R}_{1}$ by the factor $\frac{1}{k}$, and accordingly introduce the dissipation distance $\widetilde{\mathcal{R}}_{1}^{k}: L^{1}(\Gamma) \times L^{1}(\Gamma) \rightarrow$ $[0,+\infty]$,

For $k \in \mathbb{N}: \quad \widetilde{\mathcal{R}}_{1}^{k}(\tilde{z}-z):=\int_{\Gamma} \mathrm{R}_{1}^{k}(\tilde{z}-z) \mathrm{d} \mathcal{H}^{d-1}= \begin{cases}\int_{\Gamma} \frac{a_{1}}{k}|\tilde{z}-z| \mathrm{d} \mathcal{H}^{d-1} & \text { if } \tilde{z} \leq z \text { a.e. in } \Gamma, \\ +\infty & \text { otherwise, }\end{cases}$

for $k=\infty: \quad \widetilde{\mathcal{R}}_{1}^{\infty}(\tilde{z}-z):= \begin{cases}0 & \text { if } \tilde{z} \leq z \text { a.e. in } \Gamma, \\ +\infty & \text { otherwise. }\end{cases}$

We also consider the scaled mechanical energies

For $k \in \mathbb{N}: \quad \widetilde{\Phi}_{k}(u, z):=\Phi^{\mathrm{bulk}}(u)+\widetilde{\Phi}_{k}^{\text {surf }}(\llbracket u \rrbracket, z) \quad$ with $\Phi^{\mathrm{bulk}}$ from $(3.15)$ and

$$
\widetilde{\Phi}_{k}^{\text {surf }}(\llbracket u \rrbracket, z):=\int_{\Gamma}\left(I_{\mathrm{C}(x)}(\llbracket u \rrbracket)+J_{k}(\llbracket u \rrbracket, z)+I_{[0,1]}(z)-\frac{1}{k} a_{0} z\right) \mathrm{d} \mathcal{H}^{d-1}+\frac{1}{k} \mathcal{G}_{\mathrm{b}}(z),
$$

for $k=\infty: \quad \widetilde{\Phi}_{\infty}(u, z):=\Phi^{\text {bulk }}(u)+\widetilde{\Phi}_{\infty}^{\text {surf }}(\llbracket u \rrbracket, z)$ with

$$
\widetilde{\Phi}_{\infty}^{\text {surf }}(\llbracket u \rrbracket, z):=\int_{\Gamma}\left(I_{\mathrm{C}(x)}(\llbracket u \rrbracket)+J_{\infty}(\llbracket u \rrbracket, z)+I_{[0,1]}(z)\right) \mathrm{d} \mathcal{H}^{d-1},
$$

Comparing (7.2a) with (3.19), note that the terms $-a_{0} z$ and $\mathcal{G}_{\mathrm{b}}(z)$ now are also scaled by $1 / k$, so that, in the new limit energy $(7.2 \mathrm{~b})$ they are premultiplied by the factor 0 . Observe that $\widetilde{\Phi}_{\infty}$ is now defined on the space $W^{1, p}\left(\Omega \backslash \Gamma ; \mathbb{R}^{d}\right) \times L^{\infty}(\Gamma)$.

We shall refer to the systems associated with $\left(\widetilde{\mathcal{R}}_{1}^{k}, \widetilde{\Phi}_{k}\right)$, and with $\left(\widetilde{\mathcal{R}}_{1}^{\infty}, \widetilde{\Phi}_{\infty}\right)$ as the rescaled SBV-adhesive contact system, and rescaled SBV-brittle delamination system, respectively.

The ultimate reason for this new scaling resides in the semistability condition arising in the limit as $k \rightarrow \infty$. In fact, in the latter context the semistability may encompass additional information on the mechanism triggering crack initiation, as we expound below.

Remark 7.1 (The different scalings and interpretation of the semistability).

The semistability condition for the SBV-brittle system defined in Section 3.3, i.e. with the dissipation potential $\mathcal{R}_{1}$ from (3.23) and the mechanical energy $\Phi_{\mathrm{b}}$ from (3.15) and (3.22), with $\mathcal{J}_{\infty}(\llbracket u(t) \rrbracket, z(t))=0$ ultimately reads

$$
\forall \tilde{z} \in \mathcal{Z}_{\mathrm{SBV}} \text { with } \tilde{z} \leq z: \quad \mathcal{G}_{\mathrm{b}}(z(t))-\int_{\Gamma} a_{0} z(t) \mathrm{d} \mathcal{H}^{d-1} \leq \mathcal{G}_{\mathrm{b}}(\tilde{z})-\int_{\Gamma} a_{0} \tilde{z} \mathrm{~d} \mathcal{H}^{d-1}+\mathcal{R}_{1}(\tilde{z}-z(t)) .
$$

In other words, a decrease of the semistable function $z$ in time, i.e. crack growth, seems to be rather induced by the perimeter regularization than by the attempt of reducing the mechanical stresses in the specimen.

With the alternative scaling from (7.1a) and (7.2a) leading to $\widetilde{\mathcal{R}}_{1}^{\infty}$ and $\widetilde{\Phi}_{k}$, the semistability condition (3.30) of the rescaled SBV-adhesive contact system, with $k \in \mathbb{N}$ fixed, is equivalent to

$$
\begin{aligned}
\forall \tilde{z} \in \mathcal{Z}_{\mathrm{SBV}}: & \int_{\Gamma} k^{2} z_{k}(t)\left|\llbracket u_{k}(t) \rrbracket\right|^{2} \mathrm{~d} \mathcal{H}^{d-1}+\mathcal{G}_{\mathrm{b}}\left(z_{k}(t)\right)-\int_{\Gamma} a_{0} z_{k}(t) \mathrm{d} \mathcal{H}^{d-1} \\
& \leq \int_{\Gamma} k^{2} \tilde{z}\left|\llbracket u_{k}(t) \rrbracket\right|^{2} \mathrm{~d} \mathcal{H}^{d-1}+\mathcal{G}_{\mathrm{b}}(\tilde{z})-\int_{\Gamma} a_{0} \tilde{z} \mathrm{~d} \mathcal{H}^{d-1}+\mathcal{R}_{1}\left(\tilde{z}-z_{k}(t)\right) .
\end{aligned}
$$

Testing (7.4) with $\tilde{z}=0$ and exploiting that $\mathcal{R}_{1}\left(0-z_{k}(t)\right)+a_{0} \int_{\Gamma} z_{k}(t) \mathrm{d} s \leq\left(a_{0}+a_{1}\right) \mathcal{H}^{d-1}(\Gamma)=C$ we find the following estimates

$$
P\left(Z_{k}(t), \Omega\right) \leq C / \mathrm{b} \quad \text { and } \quad \int_{\Gamma} k^{2} z_{k}(t)\left|\llbracket u_{k}(t) \rrbracket\right|^{2} \mathrm{~d} s \leq C
$$


with a constant $C$ uniform for all $k \in \mathbb{N}$; again, $Z_{k}$ is the set associated to the indicator function $z_{k}$. By compactness we can thus conclude the existence of a subsequence $\left(z_{k}\right)_{k} \subset L^{\infty}((0, T) \times \Gamma) \cap \mathrm{BV}\left([0, T] ; L^{1}(\Gamma)\right)$ with $\left(z_{k}(t)\right)_{k} \subset \operatorname{SBV}(\Gamma ;\{0,1\})$ for all $t \in[0, T]$, such that $z_{k}(t) \stackrel{*}{\rightarrow} z(t)$ in $\operatorname{SBV}(\Gamma ;\{0,1\})$ for all $t \in[0, T]$. This provides the additional regularity information $z(t) \in \operatorname{SBV}(\Gamma ;\{0,1\})$ for all $t \in[0, T]$, for the function $z$ which is approximated by the sequence $\left(z_{k}\right)_{k}$, semistable for the rescaled SBV-adhesive systems. Passing to the limit $k \rightarrow \infty$ in these semistability inequalities results in

$$
\widetilde{\Phi}_{\infty}^{\text {surf }}(\llbracket u(t) \rrbracket, z(t)) \leq \widetilde{\Phi}_{\infty}^{\text {surf }}(\llbracket u(t) \rrbracket, \tilde{z})+\widetilde{\mathcal{R}}_{1}^{\infty}(\tilde{z}-z) \text { for all } \tilde{z} \in L^{\infty}(\Gamma),
$$

Clearly, (7.6) for semistable $z(t)$ and competitors $\tilde{z} \in L^{\infty}(\Gamma)$ trivially reads $0 \leq 0$ for all $\tilde{z} \leq z(t)$ and $0 \leq \infty$ otherwise. Nevertheless, at least formally, (7.6) inherits a non-trivial meaning by the very fact that it arises in the limit of (7.4). Indeed, in view of the complementarity conditions (2.11) for Signorini contact, and taking into account that $z_{k} \in\{0,1\}$, we find for every $k \in \mathbb{N}$

$$
\int_{\Gamma} k^{2} z_{k}\left|\llbracket u_{k} \rrbracket\right|^{2} \mathrm{~d} \mathcal{H}^{d-1}=\int_{\left.Z_{k} \cap|| \llbracket u_{k} \rrbracket \mid>0\right]}\left|\sigma\left(u_{k}(t), \dot{u}_{k}(t), \theta_{k}(t)\right) \mathrm{n}\right|^{2} \mathrm{~d} \mathcal{H}^{d-1} \leq C,
$$

provided that we dispose of sufficiently smooth solutions such that the term on the right-hand side makes sense. From (7.7) we read that the adhesive model accounts for the magnitude of the normal stresses. Now, under the assumption of convergence and sufficient regularity of the solutions, and taking into account that $\mathcal{H}^{d-1}\left(\left[z_{k}(t)\left|\llbracket u_{k}(t) \rrbracket\right|>0\right]\right) \rightarrow 0$, the rescaled brittle model in the limit as $k \rightarrow \infty$ therefore may contain an information of the form

$$
\int_{Z \cap \partial[|\llbracket u \rrbracket|>0]}|\sigma(u(t), \dot{u}(t), \theta(t)) \mathrm{n}|^{2} \mathrm{~d} \mathcal{H}^{d-1} .
$$

Roughly speaking, this conveys the information that, a decrease of the semistable function $z$ is not only triggered by the perimeter regularization but possibly also by the mechanical stresses.

Clearly, for every $k \in \mathbb{N}$ there exists an energetic solution $\left(u_{k}, z_{k}, w_{k}\right)$ to the the rescaled SBV-adhesive contact system. Concerning the limiting behavior of the sequence $\left(u_{k}, z_{k}, w_{k}\right)_{k}$ as $k \rightarrow \infty$, the analogue of Theorem 5.2 holds.

Theorem 7.2. Assume (3.7), (3.8) and (3.12). Let $\left(u_{k}, w_{k}, z_{k}\right)_{k}$ be a sequence of approximable solutions of the rescaled SBV-adhesive contact system, supplemented with initial data $\left(u_{k}^{0}, \theta_{k}^{0}, z_{k}^{0}\right)_{k}$ fulfilling (3.14) (4.1), (5.7), and

$$
\widetilde{\Phi}_{k}\left(u_{k}^{0}, z_{k}^{0}\right) \rightarrow \widetilde{\Phi}_{\infty}\left(u_{0}, z_{0}\right) \quad \text { as } k \rightarrow \infty .
$$

Then, there exist a (not relabeled) subsequence, and a triple $(u, w, z)$, such that convergences (4.6) hold for $\left(u_{k}, w_{k}, z_{k}\right)_{k}$ as $k \rightarrow \infty$, and $(u, w, z)$ is an energetic solution to the rescaled SBV-brittle delamination system. In addition, the analogue of (5.9) and the positivity property (4.2) hold.

Proof. We just outline how the proof for Theorem 5.2 can be adapted to the present setting, following the scheme presented in Section 3.5.

First of all, observe that the compactness argument for the sequence $\left(u_{k}, w_{k}, z_{k}\right)_{k}$ is the same as in the proof of Theorems 4.3 and 5.2: indeed, as already observed in (7.5), the sequence $\left(z_{k}\right)_{k}$ has a uniformly bounded perimeter, i.e. $\left(z_{k}\right)_{k} \subset L^{\infty}(0, T ; \operatorname{SBV}(\Gamma ;\{0,1\}))$ is bounded.

The limit passage as $k \rightarrow \infty$ in the momentum balance is obtained by adapting the arguments from Sections 5.1, which in turn hinge on the support convergence for semistable sequences. The latter can be proved arguing in the very same way as in Section 6, starting from the following basic observation: Also in the rescaled setting, the delamination parameters $z_{k}$ fulfill

$$
\mathcal{S}\left(Z_{k}\right) \leq \mathcal{S}(\tilde{Z})+\mathcal{R}_{1}\left(\tilde{z}-z_{k}\right) \text { with } \mathcal{S}(Z):=\mathrm{b} P(Z, \Gamma)-a_{0} \mathcal{H}^{m}(Z),
$$

(where $Z_{k}$ is the set of finite perimeter associated to the characteristic function $z_{k}$ ). 
The limit passage in the semistability condition follows the lines of Section 5.2, and the analogue of Proposition 5.9 holds. We just discuss how to adapt the mutual recovery sequence: in the rescaled setting, the state space for $z$ is $\mathcal{Z}=L^{\infty}(\Gamma)$. Again $\widetilde{\mathcal{R}}_{1}^{\infty}(\tilde{z}-z)<\infty$ only if $\tilde{z} \leq z$ a.e. in $\Gamma$. For such $\tilde{z} \in \mathcal{Z}$ we define the recovery sequence as follows:

$$
\tilde{z}_{k}:=\left\{\begin{array}{l}
r_{k}\left(\tilde{z}_{k}\right) \text { if } \tilde{z} \in \mathcal{Z}_{\mathrm{SBV}}, \\
r_{k}(z) \text { if } \tilde{z} \in \mathcal{Z} \backslash \mathcal{Z}_{\mathrm{SBV}},
\end{array} \quad \text { with } r_{k}(\zeta):=\zeta \mathcal{X}_{A_{k}}+z_{k}\left(1-\mathcal{X}_{A_{k}}\right)\right. \text { as in (5.27) }
$$

The construction of $\tilde{z}_{k}$ from (5.27) ensures that both $\int_{\Gamma} a_{0}\left(z_{k}-\tilde{z}_{k}\right) \mathrm{d} \mathcal{H}^{d-1}+\mathcal{R}_{1}\left(\tilde{z}_{k}-z_{k}\right) \leq\left(a_{0}+a_{1}\right) \mathcal{H}^{d-1}(\Gamma)$ and $P\left(\widetilde{Z}_{k}, \Gamma\right)=P\left(A_{k}, \Gamma\right) \leq C$. Thus we conclude that $\lim _{k \rightarrow \infty} \frac{1}{k}\left(\int_{\Gamma} a_{0}\left(z_{k}-\tilde{z}_{k}\right) \mathrm{d} \mathcal{H}^{d-1}+\mathcal{R}_{1}\left(\tilde{z}_{k}-z_{k}\right)+P\left(\widetilde{Z}_{k}, \Gamma\right)-\right.$ $\left.P\left(Z_{k}, \Gamma\right)\right)=0$. Combining these observations with (5.28) yields (5.26) for the rescaled SBV-adhesive and SBV-brittle systems.

Finally, the passages to the limit in the mechanical energy and in the weak enthalpy equality are the same as in the proofs of Theorems 4.3 and 5.2.

\section{ApPendix A}

\section{A.1. Time-discretization for the Modica-Mortola adhesive system}

In this section we outline the proof of Theorem 4.2. We perform a semi-implicit time-discretization: for a given time-step $\tau>0$, we consider the equidistant partition $\left\{t_{\tau}^{0}=0<\ldots<t_{\tau}^{j}=j \tau<\ldots<t_{\tau}^{J_{\tau}}=T\right\}$ of $[0, T]$. Hereafter, given any family $\left\{\phi^{j}\right\}_{j=1}^{J_{\tau}}$, we will denote the backward difference operator by

$$
\mathrm{D}_{t} \phi^{j}:=\frac{\phi^{j}-\phi^{j-1}}{\tau}
$$

We approximate the data $F, f$ by local means, i.e. setting $F_{\tau}^{j}:=\frac{1}{\tau} \int_{t_{\tau}^{j-1}}^{t_{\tau}^{j}} F(s) \mathrm{d} s$ and $f_{\tau}^{j}:=\frac{1}{\tau} \int_{t_{\tau}^{j-1}}^{t_{\tau}^{j}} f(s) \mathrm{d} s$ for all $j=1, \ldots, J_{\tau}$. Then, from $F_{\tau}^{j}$ and $f_{\tau}^{j}$ we define $\mathrm{F}_{\tau}^{j} \in W^{1, p}\left(\Omega \backslash \Gamma ; \mathbb{R}^{d}\right)^{*}$ as in (3.13). Furthermore, for technical reasons related to the existence proof of Problem 1 below, we need to approximate $H$ and $h$ by means of discrete data $\left\{H_{\tau}^{j}\right\}_{j=1}^{J_{\tau}},\left\{h_{\tau}^{j}\right\}_{j=1}^{J_{\tau}}$ with

$$
H_{\tau}^{j} \in W^{1,2}(\Omega)^{*}, \quad h_{\tau}^{j} \in H^{1 / 2}(\partial \Omega)^{*} \quad \text { for all } j=1, \ldots, J_{\tau},
$$

and analogously define $\mathrm{H}_{\tau}^{j} \in W^{1,2}(\Omega)^{*}$ as in (3.13). Finally, we approximate the initial datum $u_{0}$ with a sequence $\left\{u_{0, \tau}\right\} \subset W_{\Gamma_{\mathrm{D}}}^{1, \gamma}\left(\Omega \backslash \Gamma ; \mathbb{R}^{d}\right)$ (with $\gamma>\max \left\{p, \frac{2 \omega}{\omega-1}\right\}$, see Problem 1) such that

$$
\lim _{\tau \downarrow 0} \sqrt[\gamma]{\tau}\left\|e\left(u_{0, \tau}\right)\right\|_{L^{\gamma}\left(\Omega ; \mathbb{R}^{d}\right)}=0, \quad u_{0, \tau} \rightarrow u_{0} \quad \text { in } W^{1, p}\left(\Omega ; \mathbb{R}^{d}\right) \text { as } \tau \rightarrow 0 .
$$

We consider the following time-discrete approximation of the Modica-Mortola adhesive system. Therein, we add to the momentum equation the regularizing term $\tau|e(u)|^{\gamma-2} e(u)$, with $\gamma>\max \left\{p, \frac{2 \omega}{\omega-1}\right\}$ and $\omega>\frac{2 d}{d+2}$ as in (3.8b): this enables us to apply to system (A.5) and (A.6) below some existence results from the theory of pseudo-monotone operators, see the proof of Lemma A.2. Equations (A.5) and (A.6) are coupled with the time-incremental minimization Problem (A.7), whose solutions in particular fulfill the discrete flow rule (A.8). However, (A.7) contains more information than (A.8). It will enable us to prove the discrete mechanical energy inequality (A.11) and semistability (A.12) in Lemma A.4, which in turn play a crucial role in the proof of the a priori estimates of Proposition A.5. For further comments on Problem 1, we refer to Remark A.1 below.

Problem 1. Let $\gamma>\max \left\{p, \frac{2 \omega}{\omega-1}\right\}$. Given

$$
u_{\tau}^{0}=u_{0, \tau}, \quad u_{\tau}^{-1}=u_{0, \tau}-\tau \dot{u}_{0}, \quad z_{\tau}^{0}=z_{0}, \quad w_{\tau}^{0}=w_{0},
$$


find $\left\{\left(u_{\tau}^{j}, w_{\tau}^{j}, z_{\tau}^{j}\right)\right\}_{j=1}^{J_{\tau}}$, with $u_{\tau}^{j} \in W^{1, \gamma}\left(\Omega \backslash \Gamma ; \mathbb{R}^{d}\right), w_{\tau}^{j} \in W^{1,2}(\Omega \backslash \Gamma)$, and $z_{\tau}^{j} \in H^{1}(\Gamma)$, fulfilling for all $j=$ $1, \ldots, J_{\tau}$ the recursive scheme consisting of

- the (boundary-value problem for the) discrete momentum equation:

$$
\begin{gathered}
\int_{\Omega \backslash \Gamma}\left(\mathrm{DR}_{2}\left(e\left(\mathrm{D}_{t} u_{\tau}^{j}\right)\right)+\mathrm{DW}_{2}\left(e\left(u_{\tau}^{j}\right)\right)-\mathbb{B} \Theta\left(w_{\tau}^{j}\right)+\mathrm{DW}_{p}\left(e\left(u_{\tau}^{j}\right)\right)+\tau \mathrm{DW}_{\gamma}\left(e\left(u_{\tau}^{j}\right)\right)\right): e\left(v-u_{\tau}^{j}\right) \mathrm{d} x \\
+\int_{\Gamma} k z_{\tau}^{j} \llbracket u_{\tau}^{j} \rrbracket \cdot \llbracket v-u_{\tau}^{j} \rrbracket \mathrm{d} S \geq\left\langle\mathrm{F}_{\tau}^{j}, v-u_{\tau}^{j}\right\rangle
\end{gathered}
$$

for all $v \in W^{1, p}\left(\Omega \backslash \Gamma ; \mathbb{R}^{d}\right)$ with $\llbracket v(x) \rrbracket \in \mathrm{C}(x)$ for a.a. $x \in \Gamma$,

where we use the notation $\mathrm{W}_{\gamma}(e):=\frac{1}{\gamma}|e|^{\gamma-2} e: \mathbb{I}: e$, with $\mathbb{I} \in \mathbb{R}^{d \times d}$ the identity tensor;

- the (boundary-value problem for the) discrete enthalpy equation: for all $\zeta \in W^{1,2}(\Omega \backslash \Gamma)$

$$
\begin{aligned}
& \int_{\Omega \backslash \Gamma} \mathrm{D}_{t} w_{\tau}^{j} \zeta \mathrm{d} x+\int_{\Omega \backslash \Gamma} \mathcal{K}\left(e\left(u_{\tau}^{j}\right), w_{\tau}^{j}\right) \nabla w_{\tau}^{j} \cdot \nabla \zeta \mathrm{d} x+\int_{\Gamma} \eta\left(\llbracket u_{\tau}^{j-1} \rrbracket, z_{\tau}^{j}\right) \llbracket \Theta\left(w_{\tau}^{j}\right) \rrbracket \llbracket \zeta \rrbracket \mathrm{d} \mathcal{H}^{d-1} \\
& \quad=\int_{\Omega \backslash \Gamma}\left(2 \mathrm{R}_{2}\left(e\left(\mathrm{D}_{t} u_{\tau}^{j}\right)\right)-\Theta\left(w_{\tau}^{j}\right) \mathbb{B}: e\left(\mathrm{D}_{t} u_{\tau}^{j}\right)\right) \zeta \mathrm{d} x-\int_{\Gamma} \frac{\left.\zeta\right|_{\Gamma} ^{+}+\left.\zeta\right|_{\Gamma} ^{-}}{2} a_{1} \mathrm{D}_{t} z_{\tau}^{j} \mathrm{~d} \mathcal{H}^{d-1}+\left\langle\mathrm{H}_{\tau}^{j}, \zeta\right\rangle ;
\end{aligned}
$$

- the time-incremental minimization problem for the delamination parameter

$$
z_{\tau}^{j} \in \operatorname{Argmin}_{z \in H^{1}(\Gamma)}\left\{\tau \mathcal{R}_{1}\left(\frac{z-z_{\tau}^{j-1}}{\tau}\right)+\Phi_{k, m}\left(u_{\tau}^{j-1}, z\right)\right\} .
$$

Remark A.1. We highlight that the time-incremental minimization (A.7) is decoupled from equations (A.5) and (A.6): indeed, starting from $\left(u_{\tau}^{j-1}, z_{\tau}^{j-1}\right)$ one first solves (A.7) and then plugs $z_{\tau}^{j}$ in system (A.5) and (A.6), which can be handled via the theory of pseudo-monotone operators. The carefully designed coupling between (A.5)-(A.7) will be heavily exploited in the proof of Lemma A.15 below. Observe that the Euler-Lagrange equation for (A.7) yields the discrete version of the flow rule (2.14), i.e.

$$
\partial \mathscr{F}\left(z_{\tau}^{j-1} ; z_{\tau}^{j}\right)+\frac{1}{2} k\left|\llbracket u_{\tau}^{j} \rrbracket\right|^{2}+\frac{m}{2} g^{\prime}\left(z_{\tau}^{j}\right)-\frac{1}{m} \Delta z_{\tau}^{j}-a_{0}-a_{1} \ni 0 \quad \text { a.e. in } \Gamma,
$$

with $\mathscr{F}\left(z_{\tau}^{j-1} ; z\right)=\int_{\Gamma}\left(I_{(-\infty, 0]}\left(\frac{z-z_{\tau}^{j-1}}{\tau}\right)+I_{[0,1]}(z)\right) \mathrm{d} \mathcal{H}^{d-1}$ and $\partial \mathscr{F}\left(z_{\tau}^{j-1} ; \cdot\right): L^{2}(\Gamma) \rightrightarrows L^{2}(\Gamma)$ its subdifferential. However, (A.7) and (A.8) are not equivalent because of the nonconvexity of $g$, which brings about additional analytical difficulties with respect to the adhesive contact systems considered in [54,55].

Lemma A.2. Assume (3.7), (3.8), (3.12), (3.14). Then, Problem 1 admits at least one solution.

Sketch of the proof. The existence of a solution $z_{\tau}^{j}$ to (A.7) follows from the lower semicontinuity and coercivity properties of the functional $\Phi_{k, m}$, via the direct method in the Calculus of Variations. We then plug $z_{\tau}^{j}$ in (A.5) and (A.6) and prove the existence of solutions by suitably adapting the argument for [54], Lemma 7.4, where the time-discretization scheme for a thermal adhesive contact model similar to the Modica-Mortola system was analyzed.

The key idea is to apply to the elliptic system (A.5) and (A.6) a Leray-Lions type existence theorem (see e.g., [56], Chap. 2). To do so, one needs to verify that the main part of the (pseudo-monotone) operator involved in (A.5) and (A.6), is strictly monotone, and that said operator is coercive in the space $W^{1, \gamma}\left(\Omega \backslash \Gamma ; \mathbb{R}^{d}\right) \times$ $W^{1,2}(\Omega \backslash \Gamma)$ for the unknown $(u, w)$. For this coercivity property, the term $\tau \mathrm{W}_{\gamma}(e(u))=\tau|e(u)|^{\gamma-2} e(u)$ in the discrete momentum equation plays a crucial role, as it compensates the growth of the quadratic terms on the left-hand side of (A.6), with the right-hand side of (A.5). Indeed, in order to prove the coercivity of the operator underlying (A.5) and (A.6), it is necessary to test (A.6) by $w_{\tau}^{j}$, and from this derive a bound for $\left\|w_{\tau}^{j}\right\|_{W^{1,2}(\Omega \backslash \Gamma)}$. 
The related calculations involve an estimate for the term $\left|\int_{\Omega} 2 R_{2}\left(e\left(D_{t} u_{\tau}^{j}\right)\right) w_{\tau}^{j} \mathrm{~d} x\right|$, as well as the following estimate

$$
\begin{aligned}
\left|\int_{\Omega \backslash \Gamma} \Theta\left(w_{\tau}^{j}\right) \mathbb{B}: e\left(\mathrm{D}_{t} u_{\tau}^{j}\right) w_{\tau}^{j} \mathrm{~d} x\right| & \leq \frac{1}{16 \tau}\left\|w_{\tau}^{j}\right\|_{L^{2}(\Omega)}^{2}+\frac{C}{\tau} \int_{\Omega \backslash \Gamma}\left|e\left(u_{\tau}^{j}\right)-e\left(u_{\tau}^{j-1}\right)\right|^{2}\left|\left(w_{\tau}^{j}\right)^{2 / \omega}+1\right| \mathrm{d} x \\
& \leq \frac{1}{8 \tau}\left\|w_{\tau}^{j}\right\|_{L^{2}(\Omega)}^{2}+\frac{C}{\tau}\left(\left\|e\left(u_{\tau}^{j}\right)\right\|_{L^{p_{\omega}\left(\Omega ; \mathbb{R}^{d \times d}\right)}}^{p_{\omega}}+\left\|e\left(u_{\tau}^{j-1}\right)\right\|_{L^{p_{\omega}\left(\Omega ; \mathbb{R}^{d \times d}\right)}}^{p_{\omega}}+1\right) \\
& \leq \frac{1}{8 \tau}\left\|w_{\tau}^{j}\right\|_{L^{2}(\Omega)}^{2}+\frac{\tau}{8 C}\left\|u_{\tau}^{j}\right\|_{W^{1, \gamma}\left(\Omega \backslash \Gamma ; \mathbb{R}^{d}\right)}^{\gamma}+\tau C\left\|u_{\tau}^{j-1}\right\|_{W^{1, \gamma}\left(\Omega \backslash \Gamma ; \mathbb{R}^{d}\right)}^{\gamma}+C_{\tau},
\end{aligned}
$$

where we have used the placeholder $p_{\omega}:=\frac{2 \omega}{\omega-1}$. In (A.9), the first estimate is due to Hölder's inequality and to the growth condition (3.9) for $\Theta$, the second one again derives from Hölder's and Young's inequalities. For the third estimate (where $C_{\tau}$ is a positive constant depending on $\tau$ ), we have also exploited the fact that $\gamma>p_{\omega}$ which yields, via the Young inequality, that

$$
\frac{1}{\tau}\left(\left\|e\left(u_{\tau}^{j}\right)\right\|_{L^{p_{\omega}\left(\Omega ; \mathbb{R}^{d \times d}\right)}}^{p_{\omega}}+\left\|e\left(u_{\tau}^{j-1}\right)\right\|_{L^{p_{\omega}\left(\Omega ; \mathbb{R}^{d \times d}\right)}}^{p_{\omega}}\right) \leq \nu\left\|u_{\tau}^{j}\right\|_{W^{1, \gamma}\left(\Omega \backslash \Gamma ; \mathbb{R}^{d}\right)}^{\gamma}+C_{\nu} \tilde{\nu} C\left\|u_{\tau}^{j-1}\right\|_{W^{1, \gamma}\left(\Omega \backslash \Gamma ; \mathbb{R}^{d}\right)}^{\gamma}+C_{\tilde{\nu}}
$$

for every $\nu, \tilde{\nu}>0$, and suitable constants $C_{\nu}$ and $C_{\tilde{\nu}}$. Then, choosing $\nu=\frac{\tau}{8 C}$ we can absorb the second term on the right-hand side of (A.9) into the left-hand side of the discrete momentum equation tested by $u_{\tau}^{j}$, whereas the first summand is estimated by the left-hand side of (A.6) tested by $w_{\tau}^{j}$. The term involving $\left\|u_{\tau}^{j-1}\right\|_{W^{1, \gamma}\left(\Omega \backslash \Gamma ; \mathbb{R}^{d}\right)}^{\gamma}$ is estimated from the previous step. With analogous calculations one deals with the term $\left|\int_{\Omega \backslash \Gamma} 2 \mathrm{R}_{2}\left(e\left(\mathrm{D}_{t} u_{\tau}^{j}\right)\right) w_{\tau}^{j} \mathrm{~d} x\right|$. The reader is referred to the proof of ([54], Lem. 7.4) for all details. We now introduce the interpolants of the discrete solutions $\left\{\left(u_{\tau}^{j}, w_{\tau}^{j}, z_{\tau}^{j}\right)\right\}_{j=1}^{J_{\tau}}$.

Notation A.3 (Interpolants).

For $\tau>0$ fixed, the left-continuous and right-continuous piecewise constant, and the piecewise linear interpolants of the family $\left\{u_{\tau}^{j}\right\}_{j=1}^{J_{\tau}}$ are respectively the functions $\bar{u}_{\tau}, \underline{u}_{\tau}, u_{\tau}:(0, T) \rightarrow W_{\Gamma_{\mathrm{D}}}^{1, \gamma}\left(\Omega \backslash \Gamma ; \mathbb{R}^{d}\right)$ defined by

$$
\bar{u}_{\tau}(t)=u_{\tau}^{j}, \quad \underline{u}_{\tau}(t)=u_{\tau}^{j-1}, \quad u_{\tau}(t)=\frac{t-t_{\tau}^{j-1}}{\tau} u_{\tau}^{j}+\frac{t_{\tau}^{j}-t}{\tau} u_{\tau}^{j-1} \quad \text { for } t \in\left(t_{\tau}^{j-1}, t_{\tau}^{j}\right] .
$$

In the same way, we denote by $\bar{w}_{\tau}, \underline{w}_{\tau}, \bar{z}_{\tau}$ and $\underline{z}_{\tau}$, the piecewise constant interpolants of the elements $\left\{w_{\tau}^{j}\right\}_{j=1}^{J_{\tau}}$ and $\left\{z_{\tau}^{j}\right\}_{j=1}^{J_{\tau}}$, and by $w_{\tau}$ and $z_{\tau}$ the related piecewise linear interpolants. We shall also consider the interpolants $\overline{\mathrm{F}}_{\tau}$ and $\overline{\mathrm{H}}_{\tau}$ of the $J_{\tau}$-tuples $\left\{\mathrm{F}_{\tau}^{j}\right\}_{j=1}^{J_{\tau}}$ and $\left\{\mathrm{H}_{\tau}^{j}\right\}_{j=1}^{J_{\tau}}$. Finally, we use the notation $\overline{\mathrm{t}}_{\tau}$ for the left-continuous piecewise constant interpolant associated with the partition, i.e. $\overline{\mathrm{t}}_{\tau}(t)=t_{\tau}^{j}$ if $t_{\tau}^{j-1}<t \leq t_{\tau}^{j}$.

Lemma A.4. Assume (3.7), (3.8), (3.12) and (3.14). Define $\Phi_{\tau}(u, z):=\Phi_{k, m}(u, z)+\tau \int_{\Omega \backslash \Gamma} \mathrm{W}_{\gamma}(e(u)) \mathrm{d} x$. Then, for all $\tau>0$ the approximate solutions $\left(\bar{u}_{\tau}, \underline{u}_{\tau}, \bar{w}_{\tau}, \bar{z}_{\tau}, u_{\tau}, w_{\tau}, z_{\tau}\right)$ fulfill the "discrete mechanical energy" inequality

$$
\begin{gathered}
\Phi_{\tau}\left(\bar{u}_{\tau}(t), \bar{z}_{\tau}(t)\right)+\int_{0}^{\overline{\mathrm{t}}_{\tau}(t)}\left(\int_{\Omega \backslash \Gamma} 2 \mathrm{R}_{2}\left(e\left(\dot{u}_{\tau}\right)\right)+\int_{\Gamma} a_{1}\left|\dot{z}_{\tau}\right| \mathrm{d} \mathcal{H}^{d-1}\right) \mathrm{d} s \\
\leq \Phi_{\tau}\left(u_{0, \tau}, z_{0}\right)+\int_{0}^{\overline{\mathrm{t}}_{\tau}(t)}\left(\int_{\Omega \backslash \Gamma} \Theta\left(\bar{w}_{\tau}\right) \mathbb{B}: e\left(\dot{u}_{\tau}\right) \mathrm{d} x+\left\langle\overline{\mathrm{F}}_{\tau}, \dot{u}_{\tau}\right\rangle\right) \mathrm{d} s,
\end{gathered}
$$

and the "discrete semistability" for a.a. $t \in(0, T)$

$$
\Phi_{\tau}\left(\underline{u}_{\tau}(t), \bar{z}_{\tau}(t)\right) \leq \Phi_{\tau}\left(\underline{u}_{\tau}(t), \tilde{z}\right)+\mathcal{R}_{1}\left(\tilde{z}-\bar{z}_{\tau}(t)\right) \text { for all } \tilde{z} \in \mathcal{Z}_{\mathrm{MM}} \text { with } \tilde{z} \leq \underline{z}_{\tau}(t) \text { on } \Gamma \text {. }
$$


Proof. For notational simplicity we will develop the calculations in terms of the discrete solutions $\left\{\left(u_{\tau}^{j}, w_{\tau}^{j}, z_{\tau}^{j}\right)\right\}_{j=1}^{J_{\tau}}$. It follows from the time-incremental minimization (A.7) and the definition (3.17) of $\Phi_{k, m}$ that $z_{\tau}^{j} \leq z_{\tau}^{j-1}$ a.e. on $\Gamma$, and

$$
\mathcal{R}_{1}\left(z_{\tau}^{j}-z_{\tau}^{j-1}\right)+\int_{\Gamma}\left(\frac{k}{2} z_{\tau}^{j}\left|\llbracket u_{\tau}^{j-1} \rrbracket\right|^{2}-a_{0} z_{\tau}^{j}\right) \mathrm{d} \mathcal{H}^{d-1}+\mathcal{G}_{m}\left(z_{\tau}^{j}\right) \leq \int_{\Gamma}\left(\frac{k}{2} z_{\tau}^{j-1}\left|\llbracket u_{\tau}^{j-1} \rrbracket\right|^{2}-a_{0} z_{\tau}^{j-1}\right) \mathrm{d} \mathcal{H}^{d-1}+\mathcal{G}_{m}\left(z_{\tau}^{j-1}\right) .
$$

Now, let us choose in (A.5) the (admissible) test function $v=u_{\tau}^{j-1}$ and change sign in the inequality. Then, we use the elementary estimates $\mathrm{DR}_{2}\left(e\left(\mathrm{D}_{t} u_{\tau}^{j}\right)\right): e\left(u_{\tau}^{j}-u_{\tau}^{j-1}\right)=\tau 2 \mathrm{R}_{2}\left(e\left(\mathrm{D}_{t} u_{\tau}^{j}\right)\right)$ as well as $\mathrm{DW}_{n}\left(e\left(u_{\tau}^{j}\right)\right): e\left(u_{\tau}^{j}-u_{\tau}^{j-1}\right) \geq$ $\mathrm{W}_{n}\left(e\left(u_{\tau}^{j}\right)\right)-\mathrm{W}_{n}\left(e\left(u_{\tau}^{j-1}\right)\right)$ for $n=2, p, \gamma$, and $k z_{\tau}^{j} \llbracket u_{\tau}^{j} \rrbracket \cdot \llbracket u_{\tau}^{j}-u_{\tau}^{j-1} \rrbracket \geq \frac{k}{2} z_{\tau}^{j}\left|\llbracket u_{\tau}^{j} \rrbracket\right|^{2}-\frac{k}{2} z_{\tau}^{j}\left|\llbracket u_{\tau}^{j-1} \rrbracket\right|^{2}$. Thus, we obtain

$$
\begin{aligned}
& \int_{\Omega \backslash \Gamma}\left(\mathrm{W}_{2}\left(e\left(u_{\tau}^{j}\right)\right)+\mathrm{W}_{p}\left(e\left(u_{\tau}^{j}\right)\right)+\tau \mathrm{W}_{\gamma}\left(e\left(u_{\tau}^{j}\right)\right)\right) \mathrm{d} x+\tau \int_{\Omega \backslash \Gamma} 2 \mathrm{R}_{2}\left(e\left(\mathrm{D}_{t} u_{\tau}^{j}\right)\right) \mathrm{d} x+\int_{\Gamma} \frac{k}{2} z_{\tau}^{j}\left|\llbracket u_{\tau}^{j} \rrbracket\right|^{2} \\
& \left.\leq \int_{\Omega \backslash \Gamma}\left(\mathrm{W}_{2}\left(e\left(u_{\tau}^{j-1}\right)\right)+\mathrm{W}_{p}\left(e\left(u_{\tau}^{j-1}\right)\right)+\tau \mathrm{W}_{\gamma}\left(e\left(u_{\tau}^{j-1}\right)\right)\right) \mathrm{d} x+\tau \int_{\Omega \backslash \Gamma} \Theta\left(w_{\tau}^{j}\right) \mathbb{B}: e\left(\mathrm{D}_{t} u_{\tau}^{j}\right)\right) \mathrm{d} x \\
& \quad+\int_{\Gamma} \frac{k}{2} z_{\tau}^{j}\left|\llbracket u_{\tau}^{j-1} \rrbracket\right|^{2}+\tau\left\langle\mathrm{F}_{\tau}^{j}, \mathrm{D}_{t} u_{\tau}^{j}\right\rangle .
\end{aligned}
$$

Hence, we add (A.13) and (A.14), observing that the term $\int_{\Gamma} \frac{k}{2} z_{\tau}^{j}\left|\llbracket u_{\tau}^{j-1} \rrbracket\right|^{2} \mathrm{~d} \mathcal{H}^{d-1}$ cancels out. Upon summing over the index $j$, we thus arrive at the discrete mechanical energy inequality (A.11).

From (A.7) it also follows that

$$
\mathcal{R}_{1}\left(z_{\tau}^{j}-z_{\tau}^{j-1}\right)+\Phi_{k, m}\left(u_{\tau}^{j-1}, z_{\tau}^{j}\right) \leq \mathcal{R}_{1}\left(\tilde{z}-z_{\tau}^{j-1}\right)+\Phi_{k, m}\left(u_{\tau}^{j-1}, \tilde{z}\right)
$$

for all $\tilde{z} \in H^{1}(\Gamma)$ with $\tilde{z} \leq z_{\tau}^{j-1}$ on $\Gamma$, whence we immediately conclude (A.12).

As a consequence of Lemma A.4, we have the following result.

Proposition A.5 (A priori estimates).

Assume (3.7), (3.8), (3.12), and let $\left(u_{0}, z_{0}, \theta_{0}\right)$ be a triple of initial data complying with (3.14) and the semistability condition (4.3). Then, there exist constants $S^{0}>0$ and, for every $1 \leq r<\frac{d+2}{d+1}, S_{r}^{0}>0$, such that for all $\tau, m, k>0$ and for all approximate solutions $\left(\bar{u}_{\tau}, \bar{w}_{\tau}, \bar{z}_{\tau}, u_{\tau}, w_{\tau}, z_{\tau}\right)$ the following estimates hold

$$
\begin{aligned}
& \left\|\bar{u}_{\tau}\right\|_{L^{\infty}\left(0, T ; W_{\Gamma_{\mathrm{D}}}^{1, p}\left(\Omega \backslash \Gamma ; \mathbb{R}^{d}\right)\right)}+\left\|u_{\tau}\right\|_{L^{\infty}\left(0, T ; W_{\Gamma_{\mathrm{D}}}^{1, p}\left(\Omega \backslash \Gamma ; \mathbb{R}^{d}\right)\right)}+\left\|u_{\tau}\right\|_{W^{1,2}\left(0, T ; W_{\Gamma_{\mathrm{D}}}^{1,2}\left(\Omega \backslash \Gamma ; \mathbb{R}^{d}\right)\right)} \leq S_{0}, \\
& \left\|\bar{u}_{\tau}\right\|_{L^{\infty}\left(0, T ; W_{\Gamma_{\mathrm{D}}}^{1, \gamma}\left(\Omega \backslash \Gamma ; \mathbb{R}^{d}\right)\right)} \leq \frac{S_{0}}{\sqrt[\gamma]{\tau}}, \\
& \sup _{t \in[0, T]} \Phi_{\tau}\left(\bar{u}_{\tau}(t), \bar{z}_{\tau}(t)\right) \leq S_{0}, \\
& \left\|\bar{z}_{\tau}\right\|_{L^{\infty}((0, T) \times \Gamma)}+\left\|z_{\tau}\right\|_{\mathrm{BV}\left([0, T] ; L^{1}(\Gamma)\right)} \leq S_{0}, \\
& \left\|\bar{w}_{\tau}\right\|_{L^{\infty}\left(0, T ; L^{1}(\Omega)\right)}+\left\|w_{\tau}\right\|_{\mathrm{BV}\left([0, T] ; W^{1, r^{\prime}}(\Omega \backslash \Gamma)^{*}\right)} \leq S_{0}, \\
& \left\|w_{\tau}\right\|_{L^{r}\left(0, T ; W^{1, r}(\Omega \backslash \Gamma)\right)} \leq S_{r} \quad \text { for any } 1 \leq r<\frac{d+2}{d+1}
\end{aligned}
$$

where $r^{\prime}=\frac{r}{r-1}$ is the conjugate exponent of $r$. Estimates (A.15d), (A.15e) and (A.15f), respectively hold for $z_{\tau}$, $\bar{z}_{\tau}, w_{\tau}$ and $\bar{w}_{\tau}$, as well.

The proof relies on the energy inequality (A.11) and on a suitable test of the discrete enthalpy equation (A.6). The calculations are identical to those performed for ([54], Lem. 7.7), to which the reader is referred. We can now develop the 
Proof of Theorem 4.2. We follow the steps outlined in Section 3.5. However, we only detail the passage to the limit in the discrete semistability condition (A.12), since the remaining steps can be performed as in the proof of [54], Theorem 6.1, see also the arguments developed here in Section 4.

Step 0. Selection of converging subsequences. Let $\left(\tau_{j}\right)_{j}$ be a vanishing sequence of time-steps. Arguing in the very same way as in the proof of Theorem 4.3 , it can be checked that there exists a triple $(u, w, z)$ such that, up to a (not relabeled) subsequence, for the approximate solutions of Problem 1 ( $c f$. Notation A.10), the following convergences hold as $j \rightarrow \infty$ :

$$
\begin{aligned}
& u_{\tau_{j}} \rightarrow u \quad \text { in } L^{\infty}\left(0, T ; W_{\Gamma_{\mathrm{D}}}^{1, p}\left(\Omega \backslash \Gamma ; \mathbb{R}^{d}\right)\right) \cap W^{1,2}\left(0, T ; W_{\Gamma_{\mathrm{D}}}^{1,2}\left(\Omega \backslash \Gamma ; \mathbb{R}^{d}\right)\right), \\
& u_{\tau_{j}} \rightarrow u \text { in } \mathrm{C}^{0}\left([0, T] ; W_{\Gamma_{\mathrm{D}}}^{1-\epsilon, p}\left(\Omega \backslash \Gamma ; \mathbb{R}^{d}\right)\right) \\
& \bar{u}_{\tau_{j}} \stackrel{*}{\rightarrow} u \text { in } L^{\infty}\left(0, T ; W_{\Gamma_{\mathrm{D}}}^{1, p}\left(\Omega \backslash \Gamma ; \mathbb{R}^{d}\right)\right), \quad \bar{u}_{\tau_{j}} \rightarrow u \text { in } L^{\infty}\left(0, T ; W_{\Gamma_{\mathrm{D}}}^{1-\epsilon, p}\left(\Omega \backslash \Gamma ; \mathbb{R}^{d}\right)\right), \\
& \left.\bar{u}_{\tau_{j}}(t) \rightarrow u(t) \text { in } W_{\Gamma_{\mathrm{D}}}^{1-\epsilon, p}\left(\Omega \backslash \Gamma ; \mathbb{R}^{d}\right)\right) \text { for all } t \in[0, T]
\end{aligned}
$$

and for all $\epsilon \in(0,1]$. Besides, (A.15b) yields that

$$
\tau_{j}\left\|\left|e\left(\bar{u}_{\tau_{j}}\right)\right|^{\gamma-2} e\left(\bar{u}_{\tau_{j}}\right)\right\|_{L^{\gamma /(\gamma-1)}\left((0, T) \times(\Omega \backslash \Gamma) ; \mathbb{R}^{d \times d}\right)} \leq S_{0} \tau_{j}^{1 / \gamma} \rightarrow 0 \quad \text { as } \quad \tau_{j} \rightarrow 0 .
$$

Furthermore, taking into account estimate (A.15c) and the fact that $z \mapsto \Phi_{\tau}(u, z)$ has bounded sublevels in $H^{1}(\Gamma)$, and using an infinite-dimensional version of Helly's principle (see, e.g. [47], Thm. 6.1), we find that there exists $z \in L^{\infty}\left(0, T ; H^{1}(\Gamma)\right) \cap \mathrm{BV}\left([0, T] ; L^{1}(\Gamma)\right)$, with $0 \leq z(t, x) \leq 1$ for almost all $(t, x) \in(0, T) \times \Gamma$, such that

$$
\bar{z}_{\tau_{j}}, \underline{z}_{\tau_{j}} \stackrel{*}{*} z \text { in } L^{\infty}\left(0, T ; H^{1}(\Gamma)\right), \quad \bar{z}_{\tau_{j}}(t) \stackrel{*}{\rightarrow} z(t) \text { in } H^{1}(\Gamma) \text { for all } t \in[0, T] .
$$

On account of the compact embedding $H^{1}(\Gamma) \Subset L^{q}(\Gamma)$ for all $1 \leq q<\infty$, we also have

$$
\begin{aligned}
& \bar{z}_{\tau_{j}}(t) \rightarrow z(t) \text { in } L^{q}(\Gamma) \text { for all } t \in[0, T] \text { and } 1 \leq q<\infty, \text { whence } \\
& \operatorname{Var}_{\mathcal{R}_{1}}(z ;[s, t])=\lim _{\tau_{j} \rightarrow 0} \int_{s}^{t} \int_{\Gamma} a_{1}\left|\dot{z}_{\tau_{j}}(r)\right| \mathrm{d} \mathcal{H}^{d-1} \mathrm{~d} r \quad \text { for all } 0 \leq s \leq t \leq T
\end{aligned}
$$

(recall Def. (3.32) of $\operatorname{Var}_{\mathcal{R}_{1}}$ ). Thirdly, by the same tokens we conclude that there exists $w \in$ $L^{r}\left(0, T ; W^{1, r}(\Omega \backslash \Gamma)\right) \cap \mathrm{BV}\left([0, T] ; W^{1, r^{\prime}}(\Omega \backslash \Gamma)^{*}\right)$ such that

$$
\begin{array}{ll}
\bar{w}_{\tau_{j}}, w_{\tau_{j}} \rightarrow w & \text { in } L^{r}\left(0, T ; W^{1, r}(\Omega \backslash \Gamma)\right), \\
\bar{w}_{\tau_{j}}, w_{\tau_{j}} \rightarrow w & \text { in } L^{r}\left(0, T ; W^{1-\epsilon, r}(\Omega \backslash \Gamma)\right) \cap L^{q}\left(0, T ; L^{1}(\Omega)\right) \forall \epsilon \in(0,1], 1 \leq q<\infty, \\
w_{\tau_{j}}(t) \stackrel{*}{\rightarrow} w(t) \quad \text { in } W^{1, r^{\prime}}(\Omega \backslash \Gamma)^{*} \text { for all } t \in[0, T] .
\end{array}
$$

Finally, let us observe that, thanks to (A.19) and (A.20), we have $\mathcal{G}_{m}(z(t)) \leq \liminf _{\tau_{j} \rightarrow 0} \mathcal{G}_{m}\left(\bar{z}_{\tau_{j}}(t)\right)$ for all $t \in[0, T]$. Therefore, also in view of the previous convergences (A.16)-(A.18), we conclude

$$
\Phi_{k, m}(u(t), z(t)) \leq \liminf _{\tau_{j} \rightarrow 0} \Phi_{\tau_{j}}\left(\bar{u}_{\tau_{j}}(t), \bar{z}_{\tau_{j}}(t)\right) .
$$

Step 1. Momentum equation. Relying on convergences (A.16)-(A.22), as well as on the convergence $\overline{\mathrm{F}}_{\tau} \rightarrow \mathrm{F}$ in $L^{2}\left(0, T ; W^{1,2}\left(\Omega \backslash \Gamma ; \mathbb{R}^{d}\right)^{*}\right) \cap W^{1,1}\left(0, T ; W^{1, p}\left(\Omega \backslash \Gamma ; \mathbb{R}^{d}\right)^{*}\right)$, and arguing in the very same way as in the proof of Step 1 for Theorem 4.3, it is possible to pass to the limit in the discrete momentum inclusion (A.5) for the approximating solutions. For this, we also need to construct suitable recovery sequences (see [54], (8.7b), and also [65]) for the test functions of the weak formulation (3.29a) of the momentum inclusion in the adhesive case. Hence we conclude that $(u, w, z)$ comply with (3.29a). 
Step 2. Semistability condition. Like in the proof of Theorems 4.3 and 5.2, in order to show that the pair $(u, z)$ fulfills the semistability condition (3.30), we need to verify for the sequence $\left(\underline{u}_{\tau_{j}}, \bar{z}_{\tau_{j}}\right)_{j}$ the mutual recovery sequence condition. Viz., that for all $t \in[0, T]$ and for all $\tilde{z} \in \mathcal{Z}_{\mathrm{MM}}=H^{1}(\Gamma)$ with $\mathcal{R}_{1}(\tilde{z}-z)<\infty$, there is a sequence $\left(\tilde{z}_{j}\right)_{j}$ (t-dependence omitted) so that $\tilde{z}_{j} \rightarrow \tilde{z}$ in $H^{1}(\Gamma)$ as $j \rightarrow \infty$ and

$$
\limsup _{\tau_{j} \rightarrow 0}\left(\Phi_{\tau_{j}}\left(\underline{u}_{\tau_{j}}(t), \tilde{z}_{j}\right)+\mathcal{R}_{1}\left(\tilde{z}_{j}-\bar{z}_{\tau_{j}}(t)\right)-\Phi_{\tau_{j}}\left(\underline{u}_{\tau_{j}}(t), \bar{z}_{\tau_{j}}(t)\right)\right) \leq \Phi_{k, m}(u(t), \tilde{z})+\mathcal{R}_{1}(\tilde{z}-z(t))-\Phi_{k, m}(u(t), z(t)) .
$$

Notice that, for (A.25) to hold, it is necessary that $\tilde{z}_{j} \in H^{1}(\Gamma) \cap L^{\infty}(\Gamma)$ and

$$
0 \leq \tilde{z}_{j} \leq \underline{z}_{\tau_{j}}(t) \leq 1 \quad \text { a.e. in } \Gamma \text {. }
$$

For $\left(\tilde{z}_{j}\right)_{j}$, we use the construction from the proof of [64], Theorem 3.14, and set

$$
\tilde{z}_{j}:=\min \left\{\left(\tilde{z}-\delta_{j}\right)^{+}, \underline{z}_{\tau_{j}}(t)\right\}=\left\{\begin{array}{l}
\tilde{z}-\delta_{j} \text { if }\left(\tilde{z}-\delta_{j}\right)^{+} \leq \underline{z}_{\tau_{j}}(t), \\
\underline{z}_{\tau_{j}}(t) \text { if }\left(\tilde{z}-\delta_{j}\right)^{+}>\underline{z}_{\tau_{j}}(t)
\end{array} \quad \text { with } \delta_{j}:=\left\|\underline{z}_{\tau_{j}}(t)-z(t)\right\|_{L^{2}(\Gamma)}^{1 / 2} .\right.
$$

Clearly, $\left(\tilde{z}_{j}\right)_{j}$ fulfill (A.26). In view of (A.20), $\delta_{j} \rightarrow 0$ as $j \rightarrow \infty$. Let us now verify that $\left(\tilde{z}_{j}\right)_{j}$ complies with (A.25). First of all, the very same argument as in [64] yields that $\left(\tilde{z}_{j}\right)_{j} \subset H^{1}(\Gamma)$, and that $\tilde{z}_{j} \rightarrow \tilde{z}$ in $H^{1}(\Gamma)$, hence $\tilde{z}_{j} \rightarrow \tilde{z}$ in $L^{q}(\Gamma)$ for all $1 \leq q<\infty$. Therefore, on account of (A.19) we immediately have that $\lim _{\tau_{j} \rightarrow 0} \mathcal{R}_{1}\left(\tilde{z}_{j}-\bar{z}_{\tau_{j}}(t)\right)=\mathcal{R}_{1}(\tilde{z}-z(t))$. Furthermore, also in view of (A.17) we have

$$
\left\{\begin{array}{l}
\left.\lim _{\tau_{j} \rightarrow 0} \int_{\Gamma} \frac{k}{2}\left(\tilde{z}_{j}-\bar{z}_{\tau_{j}}(t)\right)|\llbracket| \underline{u}_{\tau_{j}}(t) \rrbracket\right|^{2} \mathrm{~d} \mathcal{H}^{d-1}=\int_{\Gamma} \frac{k}{2}(\tilde{z}-z(t))|\llbracket u(t) \rrbracket|^{2} \mathrm{~d} \mathcal{H}^{d-1}, \\
\lim _{\tau_{j} \rightarrow 0} \int_{\Gamma} a_{0}\left(\bar{z}_{\tau_{j}}(t)-\tilde{z}_{j}\right) \mathrm{d} S=\int_{\Gamma} a_{0}(z(t)-\tilde{z}) \mathrm{d} S \\
\lim _{\tau_{j} \rightarrow 0} \int_{\Gamma} \frac{m}{2}\left(g\left(\tilde{z}_{j}\right)-g\left(\bar{z}_{\tau_{j}}(t)\right)\right) \mathrm{d} \mathcal{H}^{d-1}=\int_{\Gamma} \frac{m}{2}(g(\tilde{z})-g(z(t))) \mathrm{d} \mathcal{H}^{d-1}
\end{array}\right.
$$

with $g(z)=z^{2}(1-z)^{2}$. Repeating the very same calculations as for ([64], Thm. 3.14), it can also be checked that

$$
\limsup _{\tau_{j} \rightarrow 0} \int_{\Gamma} \frac{1}{2 m}\left(\left|\nabla \tilde{z}_{j}\right|^{2}-\left|\nabla \bar{z}_{\tau_{j}}(t)\right|^{2}\right) \mathrm{d} \mathcal{H}^{d-1} \leq \int_{\Gamma} \frac{1}{2 m}\left(|\nabla \tilde{z}|^{2}-|\nabla z(t)|^{2}\right) \mathrm{d} \mathcal{H}^{d-1}
$$

Then, (A.25) ensues from (A.28) and (A.29).

Step 3. Mechanical energy inequality. The mechanical energy inequality (3.31) can be obtained via the very same lower semicontinuity argument as in Step 3 of the proof of Theorem 4.3.

Steps 4. Enthalpy inequality. The previously proved convergences, as well as the fact that $\overline{\mathrm{H}}_{\tau_{j}} \rightarrow \mathrm{H}$ in $L^{1}\left(0, T ; W^{1, r}\left(\Omega \backslash \Gamma \mathbb{R}^{d}\right)^{*}\right)$, allow us to take the limit of the discrete enthalpy equation (A.6) with positive test functions $\zeta$. Arguing in the very same way as in Step 4 of the proof of Theorem 4.3, we prove the weak enthalpy inequality (3.33).

Positivity of the temperature. Repeating the comparison argument from the proof of Lemma 7.4 from [54], (the related calculations rely in particular on (3.10) in the present paper), it is possible to show that, if there exists $\theta^{*}>0$ such that $\theta_{0}(x) \geq \theta^{*}$ for almost all $x \in \Omega$, then

$$
w(x, t) \geq \frac{1}{C^{\prime} T+h\left(\theta^{*}\right)+1} \quad \text { for a.a. }(x, t) \in \Omega \times(0, T),
$$

where the constant $C^{\prime}$ only depends on the problem data. Then, (4.2) ensues.

We are now in the position to briefly sketch the proof of Proposition 3.14: estimates (3.48)-(3.53) follow by lower semicontinuity arguments. Indeed, we start from the time-discretization of the Modica-Mortola system. For the related approximate solutions, the estimates of Proposition A.5 hold, with a constant independent of 
the time-step $\tau$, and of the parameters $m$ and $k$. In view of the convergences (A.16)-(A.24) of the approximate solutions, such estimates are inherited by the approximable energetic solutions of the Modica-Mortola delamination system. This yields the bounds (3.48)-(3.53), with a constant independent of $m$ and $k$. Then, the convergences stated in Theorem 4.3 and again lower semicontinuity arguments ensure that (3.48)-(3.53) are also valid for the approximable energetic solutions of the SBV-adhesive system, uniformly w.r.t. the parameter $k$. This concludes the proof.

\section{A.2. Tools from the theory of BV-functions}

In order to make this paper as self-contained as possible, below we collect all the measure-theoretic definitions and tools from the theory of BV-functions which have been used. In what follows, $D \subset \mathbb{R}^{m}$ will denote a bounded set and $\mathcal{X}_{D}$, with $\mathcal{X}_{D}(x)=1$ if $x \in D, \mathcal{X}_{D}(x)=0$ otherwise, its characteristic function. In Sections 1-6, all the statements below apply to $D=\Gamma$ and $m=d-1$.

Definition A.6 (BV-functions and sets of finite perimeter [4], Defs. 3.4, 3.35, Prop. 3.6). Let $D$ be an open subset in $\mathbb{R}^{m}$ and $v \in L_{\mathrm{loc}}^{1}(D)$. The variation $V(v, D)$ of $v$ in $D$ is defined by

$$
V(v, D):=\sup \left\{\int_{D} v \operatorname{div} \varphi \mathrm{d} x \mid \varphi \in \mathrm{C}_{c}^{1}(D)^{m},\|\varphi\|_{L^{\infty}(D)} \leq 1\right\} .
$$

Here, $\mathrm{C}_{c}^{1}(D)^{m}$ is the space of continuously differentiable functions $\phi: D \rightarrow \mathbb{R}^{m}$ with compact support in $D$. For $v \in L^{1}(D)$ it holds $v \in \mathrm{BV}(D)$ if and only if $V(v, D)<\infty$ and then, $V(v, D)=|\mathrm{D} v|(D)$.

Let $E$ be an $\mathcal{L}^{m}$-measurable subset of $\mathbb{R}^{m}$. The perimeter of $E$ in $D$, denoted by $P(E, D)$, is the variation of the characteristic function $\mathcal{X}_{E}$ in $D$, i.e.

$$
P(E, D)=V\left(\mathcal{X}_{E}, D\right) \text {. }
$$

We say that $E$ is a set of finite perimeter in $D$ if $P(E, D)<\infty$.

Theorem A.7 ([4], Thm. 3.36).

For any set $E$ of finite perimeter in $D$ the distributional derivative $\mathrm{D} \mathcal{X}_{E}$ is an $\mathbb{R}^{m}$-valued finite Radon measure in D. Moreover, $P(E, D)=\left|\mathrm{D} \mathcal{X}_{E}\right|(D)$ and a generalized Gauss-Green formula holds:

$$
\int_{E} \operatorname{div} \varphi \mathrm{d} x=-\int_{D} \nu_{E} \cdot \varphi \mathrm{d}\left|\mathrm{D} \mathcal{X}_{E}\right| \quad \text { for all } \mathrm{C}_{c}^{1}(D)^{m}
$$

where $\mathrm{D} \mathcal{X}_{E}=\nu_{E}\left|\mathrm{D} \mathcal{X}_{E}\right|$ is the polar decomposition of $\mathrm{D} \mathcal{X}_{E}$, i.e. $\nu_{E} \in L^{1}\left(D,\left|\mathrm{D} \mathcal{X}_{E}\right|\right)^{m}$ is the Radon-Nikodým density for the measure $\mathrm{D} \mathcal{X}_{E}$ with respect to the measure $\left|\mathrm{D} \mathcal{X}_{E}\right|$.

Proposition A.8 ([4], Prop. 3.38, Properties of the perimeter).

1. The mapping $E \mapsto P(E, D)$ is lower semicontinuous w.r.t. local convergence in measure in $D$.

2. The mapping $E \mapsto P(E, D)$ is local, i.e. $P(E, D)=P(F, D)$ whenever $\mid D \cap((E \backslash F) \cup(F \backslash E) \mid=0$.

3. It holds $P(E, D)=P\left(\mathbb{R}^{m} \backslash E, D\right)$ and

$$
P(E \cup F, D)+P(E \cap F, D) \leq P(E, D)+P(F, D) .
$$

Theorem A.9 ([4], Thm. 3.40, Coarea formula in BV). Let $v \in L_{\mathrm{loc}}^{1}(D)$ and $D \subset \mathbb{R}^{m}$ an open set. For the variation of $v$ in $D$ it holds

$$
V(v, D)=\int_{-\infty}^{\infty} P(\{x \in D \mid v(x)>t\}, D) \mathrm{d} t .
$$


In particular, if $v \in \mathrm{BV}(D)$ the set $\{v>t\}$ has finite perimeter for $\mathcal{L}^{1}$-a.a. $t \in \mathbb{R}$ and

$$
|\mathrm{D} v|(B)=\int_{-\infty}^{\infty}\left|\mathrm{D} \mathcal{X}_{\{v>t\}}\right|(B) \mathrm{d} t, \quad \mathrm{D} v(B)=\int_{-\infty}^{\infty} \mathrm{D} \mathcal{X}_{\{v>t\}}(B) \mathrm{d} t
$$

for any Borel set $B \subset D$.

Definition A.10 ([4], Def. 3.54, Reduced boundary).

Let $E$ be an $\mathcal{L}^{m}$-measurable subset of $\mathbb{R}^{m}$ and $D$ the largest open set such that $E$ is locally of finite perimeter in $D$. We define the reduced boundary $\mathfrak{F} E$ as the collection of all points $x \in \operatorname{supp}\left|D \mathcal{X}_{E}\right| \cap D$ such that the limit

$$
\nu_{E}(x):=\lim _{\varrho \rightarrow 0} \frac{\mathrm{D} \mathcal{X}_{E}\left(B_{\varrho}(x)\right)}{\left|\mathrm{D} \mathcal{X}_{E}\right|\left(B_{\varrho}(x)\right)}
$$

exists in $\mathbb{R}^{m}$ and satisfies $\left|\nu_{E}(x)\right|=1$. The function $\nu_{E}: \mathfrak{F} E \rightarrow \mathbb{S}^{m-1}$ is called the generalized inner normal to $E ;$ here, $\mathbb{S}^{m-1}$ denotes the unit sphere in $\mathbb{R}^{m}$.

Definition A.11 ([4], Def. 3.60, Points of density $t$, essential boundary).

For all $t \in[0,1]$ and every $\mathcal{L}^{m}$-measurable set $E \subset \mathbb{R}^{m}$ we denote by $E^{t}$ the set

$$
\left\{x \in \mathbb{R}^{m} \mid \lim _{\varrho \rightarrow 0} \frac{\mathcal{L}^{m}\left(E \cap B_{\varrho}(x)\right)}{\mathcal{L}^{m}\left(B_{\varrho}(x)\right)}=t\right\}
$$

of all points where $E$ has density $t$. We denote by $\partial^{*} E$ the essential boundary of $E$, i.e. the set $\mathbb{R}^{m} \backslash\left(E^{0} \cup E^{1}\right)$ of points where the density is either 0 or 1 . Moreover, $E^{1}$ can be considered as the measure-theoretic interior and $E^{0}$ as the measure-theoretic exterior of the set $E$.

Corollary A.12. The measure-theoretic interior has the following properties:

1. Let $N \subset D$ with $\mathcal{L}^{m}(N)=0$. Then $N^{1}=\emptyset$ and $(D \backslash N)^{1}=D^{1}$.

2. Let $A \subset B \subset D$. Then $A^{1} \subset B^{1} \subset D^{1}$.

The next theorem, which is due to Federer, states that $\mathfrak{F} E$ is the relevant part of the boundary, since $D \backslash\left(E^{0} \cup\right.$ $\mathfrak{F} E \cup E^{1}$ ) is a $\mathcal{H}^{m-1}$-negligible set.

Theorem A.13 ([4], Thm. 3.61, Federer).

Let $E$ be a set of finite perimeter in $D$. Then

$$
\mathfrak{F} E \cap D \subset E^{1 / 2} \subset \partial^{*} E \quad \text { and } \quad \mathcal{H}^{m-1}\left(D \backslash\left(E^{0} \cup \mathfrak{F} E \cup E^{1}\right)\right)=0 .
$$

In particular, $E$ has density either 0 or $1 / 2$ or 1 at $\mathcal{H}^{m-1}$-a.a. $x \in D$ and $\mathcal{H}^{m-1}$-a.a. $x \in \partial^{*} E \cap D$ belongs to $\mathfrak{F} E$.

Definition A.14 ([4], Def. 3.63, Approximate limit).

Let $v \in L_{\mathrm{loc}}^{1}(D)^{m}$. We say that $v$ has an approximate limit at $x \in D$ if there exists $\bar{v} \in \mathbb{R}^{m}$ such that

$$
\lim _{\varrho \rightarrow 0} f_{B_{\varrho}(x)}|v(y)-\bar{v}| \mathrm{d} y=0 .
$$

The set $S_{v}$ of points where this property does not hold is called the approximate discontinuity set. For any $x \in D \backslash S_{v}$ the vector $\bar{v}$, uniquely determined by (A.40), is called approximate limit of $v$ at $x$ and denoted by $\tilde{v}(x)$. 
We will use the notation

$$
B_{\varrho}^{+}(x, \nu):=\left\{y \in B_{\varrho}(x) \mid\langle y-x, \nu\rangle>0\right\}, \quad B_{\varrho}^{-}(x, \nu):=\left\{y \in B_{\varrho}(x) \mid\langle y-x, \nu\rangle<0\right\} .
$$

Definition A.15 ([4], Def. 3.67, Approximate jump points).

Let $v \in L_{\text {loc }}^{1}(D)^{m}$ and $x \in D$. We say that $x$ is an approximate jump point of $v$ if there exist $a, b \in \mathbb{R}^{m}$ and $\nu \in \mathbb{S}^{m-1}$ so that $a \neq b$ and

$$
\lim _{\varrho \rightarrow 0} f_{B_{\varrho}^{+}(x, \nu)}|v(y)-a| \mathrm{d} y=0, \quad \lim _{\varrho \rightarrow 0} f_{B_{\varrho}^{-}(x, \nu)}|v(y)-b| \mathrm{d} y=0 .
$$

The triple $(a, b, \nu)$, uniquely determined by (A.41) up to a permutation of $(a, b)$ and a change of sign of $\nu$, is denoted by $\left(v^{+}, v^{-}, \nu_{v}(x)\right)$. The set of approximate jump points of $v$ is denoted by $\mathrm{J}_{v}$.

Definition A.16 ([4], Def. 2.57, Rectifiable sets).

Let $E \subset \mathbb{R}^{m}$ be an $\mathcal{H}^{k}$-measurable set. The set $E$ is countably $k$-rectifiable if there exist countably many Lipschitz functions $f_{i}: \mathbb{R}^{k} \rightarrow \mathbb{R}^{m}$ such that

$$
E \subset \cup_{i=0}^{\infty} f_{i}\left(\mathbb{R}^{k}\right)
$$

$E$ is countably $\mathcal{H}^{k}$-rectifiable if there are countably many Lipschitz functions $f_{i}: \mathbb{R}^{k} \rightarrow \mathbb{R}^{m}$ so that

$$
\mathcal{H}^{k}\left(E \backslash \cup_{i=0}^{\infty} f_{i}\left(\mathbb{R}^{k}\right)\right)=0 .
$$

Clearly, $k$-rectifiability implies $\mathcal{H}^{k}$-rectifiability.

Theorem A.17 ([4], Thm. 3.59, De Giorgi).

Let $E$ be an $\mathcal{L}^{m}$-measurable subset of $\mathbb{R}^{m}$. Then $\mathfrak{F} E$ is countably $(m-1)$-rectifiable and $\left|\mathrm{D} \mathcal{X}_{E}\right|=\mathcal{H}^{m-1}\lfloor\mathfrak{F} E$.

By the Besicovitch derivation theorem ([4], Thm. 2.22) one obtains that for any set of finite perimeter $E$ that $\left|\mathrm{D} \mathcal{X}_{E}\right|$ is concentrated on $\mathfrak{F} E$. Hence, in this case, by Theorem A.17 the Gauss-Green formula (A.33) can be rewritten as

$$
\int_{E} \operatorname{div} \varphi \mathrm{d} x=-\int_{\mathfrak{F} E} \nu_{E} \cdot \varphi \mathrm{d} \mathcal{H}^{m-1} \quad \text { for all } \varphi \in C_{c}^{1}(D)^{m} .
$$

Due to Theorem A.17 the perimeter of $E$ can be computed by

$$
P(E, D)=\mathcal{H}^{m-1}\left(D \cap \partial^{*} E\right)=\mathcal{H}^{m-1}\left(D \cap E^{1 / 2}\right) .
$$

This can be used to rewrite the coarea formula (A.35) using the essential boundary of level sets

$$
|\mathrm{D} u|(B)=\int_{-\infty}^{\infty} \mathcal{H}^{m-1}\left(B \cap \partial^{*}\{u>t\}\right) \mathrm{d} t \quad \text { for all Borel sets } B \subset D .
$$

Theorem A.18 ([4], Thm. 3.77, Traces on interior rectifiable sets).

Let $v \in \mathrm{BV}(D)^{m}$ and let $\Gamma \subset D$ be a countably $\mathcal{H}^{m-1}$-rectifiable set oriented by $\nu$. Then, for $\mathcal{H}^{m-1}$-a.a. $x \in \Gamma$ there exist $v_{\Gamma}^{+}(x), v_{\Gamma}^{-}(x) \in \mathbb{R}^{m}$ such that

$$
\lim _{\varrho \rightarrow 0} f_{B_{\varrho}^{+}(x, \nu(x))}\left|v(y)-v_{\Gamma}^{+}(x)\right| \mathrm{d} y=0, \quad \lim _{\varrho \rightarrow 0} f_{B_{\varrho}^{-}(x, \nu(x))}\left|v(y)-v_{\Gamma}^{-}(x)\right| \mathrm{d} y=0 .
$$

Moreover, $\mathrm{D} v\left\lfloor\Gamma=\left(v_{\Gamma}^{+}-v_{\Gamma}^{-}\right) \otimes \nu \mathcal{H}^{m-1}\lfloor\Gamma\right.$. 
Acknowledgements. We would like to thank T. Roubíček for proposing this problem to us and for fruitful discussions. R.R. gratefully acknowledges the kind hospitality of the Weierstrass Institute for Applied Analysis and Stochastics, where most of this research was carried out. M.T. gratefully acknowledges the kind hospitality of the Universities of Pavia and Brescia during several stays in the course of this research. M.T. also wants to thank J.A. Griepentrog, D. Knees and G. Dal Maso for some inspiring discussions about Section 6. Finally, we are grateful to the anonymous referee for plenty of useful remarks and suggestions.

\section{REFERENCES}

[1] V. Acary and B. Brogliato, Numerical Methods for Nonsmooth Dynamical Systems. Springer (2008).

[2] G. Alberti, Variational models for phase transitions, an approach via $\Gamma$-convergence, in Calculus of variations and partial differential equations (Pisa 1996). Springer (2000) 95-114.

[3] L. Ambrosio and G. Dal Maso, A general chain rule for distributional derivatives. In vol. 108. Proc. of Amer. Math. Soc. (1990) 691-702.

[4] L. Ambrosio, N. Fusco and D. Pallara, Functions of Bounded Variation and Free Discontinuity Problems. Oxford University Press (2005).

[5] H. Attouch, Variational convergence for functions and operators. Appl. Math. Ser. Pitman (Advanced Publishing Program), Boston, MA (1984).

[6] J.F. Bell, Mechanics of Solids. Vol. 1: The Experimental Foundations of Solid Mechanics. Springer (1984).

[7] L. Boccardo and T. Gallouët, Non-linear elliptic and parabolic equations involving measure data. J. Funct. Anal. 87 (1989) 149-169.

[8] E. Bonetti, G. Bonfanti and R. Rossi, Global existence for a contact problem with adhesion. Math. Meth. Appl. Sci. 31 (2008) 1029-1064.

[9] E. Bonetti, G. Bonfanti and R. Rossi, Thermal effects in adhesive contact: Modelling and analysis. Nonlinearity 22 (2009) $2697-2731$.

[10] E. Bonetti, G. Schimperna and A. Segatti, On a doubly nonlinear model for the evolution of damaging in viscoelastic materials. J. Differ. Equ. 218 (2005) 91-116.

[11] B. Bourdin, G. Francfort and J.-J. Marigo, The variational approach to fracture. J. Elasticity 91 (2008) 5-148.

[12] S. Campanato, Proprietà di hölderianità di alcune classi di funzioni. Annali della Scuola Normale Superiore di Pisa 17 (1963) $175-188$.

[13] S. Campanato, Proprietà di una famiglia di spazi funzionali. Annali della Scuola Normale Superiore di Pisa 18 (1964) $137-160$.

[14] V. Caselles, A. Chambolle and M. Novaga, Regularity for solutions of the total variation denoising problem. Revista Matemática Iberoamericana 27 (2011) 729-1098.

[15] G. Dal Maso, G. Francfort and R. Toader, Quasistatic crack growth in nonlinear elasticity. Arch. Rat. Mech. Anal. 176 (2005) $165-225$.

[16] G. Dolzmann, N. Hungerbühler and S. Müller, Uniqueness and maximal regularity for nonlinear elliptic systems of $n$-Laplace type with measure valued right hand side. J. für die reine und angewandte Mathematik 520 (2000) 1-35.

[17] L.C. Evans and R.F. Gariepy, Measure Theory and Fine Properties of Functions. CRC Press (1992).

[18] H. Federer, Geometric Measure Theory. Springer (1969).

[19] E. Feireisl, Dynamics of viscous compressible fluids, Vol. 26 of Oxford Lect. Ser. Math. Appl. Oxford University Press, Oxford (2004).

[20] E. Feireisl, Mathematical theory of compressible, viscous, and heat conducting fluids. Comput. Math. Appl. 53 (2007) 461-490.

[21] E. Feireisl, H. Petzeltová and E. Rocca, Existence of solutions to a phase transition model with microscopic movements. Math. Methods Appl. Sci. 32 (2009) 1345-1369.

[22] E. Feireisl, M. Frémond, E. Rocca and G. Schimperna, A new approach to non-isothermal models for nematic liquid crystals. Arch. Ration. Mech. Anal. 205 (2012) 651-672.

[23] I. Fonseca and N. Fusco, Regularity results for anisotropic image segmentation models. Annali della Scuola Normale Superiore di Pisa 24 (1997) 463-499.

[24] G. Francfort and A. Mielke, Existence results for a class of rate-independent material models with nonconvex elastic energies. J. reine angew. Math. 595 (2006) 55-91.

[25] F. Freddi and M. Frémond, Damage in domains and interfaces: A coupled predictive theory. J. Mech. Mat. Struct. 1 (2006) $1205-1233$.

[26] M. Frémond, Contact with adhesion, in Topics in Nonsmooth Mechanics. Edited by J.J. Moreau, P.D. Panagiotopoulos and G. Strang. Birkhäuser (1988) 157-186.

[27] M. Frémond, Non-Smooth Thermomechanics. Springer-Verlag, Berlin, Heidelberg (2002).

[28] M. Frémond and B. Nedjar, Damage, gradient of damage and principle of virtual power. Int. J. Solids Struct. 33 (1996) 1083-1103.

[29] Y.C. Fung, Foundations of solid mechanics. Prentice-Hall, Inc. (1965).

[30] P. Gérard, Microlocal defect measures. Commun. Partial Differ. Equ. 16 (1991) 1761-1794. 
[31] A. Giacomini, Ambrosio-Tortorelli approximation of quasi-static evolution of brittle fracture. Calc. Var. Partial Differ. Equ. 22 (2005) 129-172.

[32] M. Giaquinta, Multiple Integrals in the Calculus of Variations and Nonlinear Elliptic Systems. Princeton University Press (1983).

[33] E. Giusti, Minimal Surfaces and Functions of Bounded Variation. Birkhäuser, Boston (1984).

[34] J.A. Griepentrog, Linear elliptic boundary value problems with non-smooth data: Campanato spaces of functionals. Math. Nachrichten 243 (2002) 19-42.

[35] J.A. Griepentrog and L. Recke, Linear elliptic boundary value problems with non-smooth data: Normal solvability on SobolevCampanato spaces. Math. Nachrichten 225 (2001) 39-74.

[36] B. Halphen and Q.S. Nguyen, Sur les matériaux standards généralisés. J. Mécanique 14 (1975) 39-63.

[37] C.O. Horgan and J.K Knowles, The effect of nonlinearity on a principle of Saint-Venant type. J. Elasticity 11 (1981) $271-291$.

[38] A.D. Ioffe and V.M. Tihomirov, Theory of extremal problems, vol. 6 of Stud. Math. Appl. Translated from the Russian by Karol Makowski. North-Holland Publishing Co., Amsterdam (1979).

[39] D. Knees, A. Mielke and C. Zanini, On the inviscid limit of a model for crack propagation. Math. Models Methods Appl. Sci. 18 (2008) 1529-1569.

[40] J.K. Knowles, The finite anti-plane shear near the tip of a crack for a class of incompressible elastic solids. Int. J. Fracture $\mathbf{1 3}$ (1977) 611-639.

[41] M. Kočvara, A. Mielke and T. Roubíček, A rate-independent approach to the delamination problem. Math. Mech. Solids 11 (2006) 423-447.

[42] J.L. Lewis, Uniformly fat sets. Trans. Amer. Math. Soc. 308 (1988) 177-196.

[43] F. Maggi, Sets of finite perimeter and geometric variational problems. Cambridge (2012).

[44] M. Marcus and V.J. Mizel, Absolute continuity on tracks and mappings of Sobolev spaces. Arch. Rational Mech. Anal. 45 (1972) 294-320.

[45] A. Mielke, Evolution in rate-independent systems, in vol. 2 of Handbook Differ. Equ. Evol. Equ., edited by C.M. Dafermos and E. Feireisl. Elsevier B.V., Amsterdam (2005) 461-559.

[46] A. Mielke and T. Roubíček, Rate-independent damage processes in nonlinear elasticity. Math. Models Methods Appl. Sci. 16 (2006) 177-209.

[47] A. Mielke and F. Theil, On rate-independent hysteresis models. Nonlin. Differ. Equ. Appl. 11 (2004) $151-189$.

[48] A. Mielke, T. Roubíček and U. Stefanelli, $\Gamma$-limits and relaxations for rate-independent evolutionary problems. Calc. Var. Partial Differ. Equ. 31 (2008) 387-416.

[49] A. Mielke, T. Roubíček and M. Thomas, From damage to delamination in nonlinearly elastic materials at small strains. J. Elasticity 109 (2012) 235-273.

[50] L. Modica, The gradient theory of phase transitions and the minimal interface criterion. Arch. Rational Mech. Anal. 98 (1987) 123-142.

[51] L. Modica and S. Mortola, Un esempio di $\Gamma$-convergenza. Boll. U. Mat. Ital. B 14 (1977) 285-299.

[52] J. Naumann, On the existence of weak solutions to the equations of non-stationary motion of heat-conducting incompressible viscous fluids. Math. Methods Appl. Sci. 29 (2006) 1883-1906.

[53] M. Negri and C. Ortner, Quasi-static crack propagation by Griffith's criterion. Math. Models Methods Appl. Sci. 18 2008 1895-1925.

[54] R. Rossi and T. Roubíček, Thermodynamics and analysis of rate-independent adhesive contact at small strains. Nonlinear Anal. 74 (2011) 3159-3190.

[55] R. Rossi and T. Roubíček, Adhesive contact delaminating at mixed mode, its thermodynamics and analysis. Interfaces Free Bound. 14 (2013) 1-37.

[56] T. Roubíček, Nonlinear Partial Differential Equations with Applications. Birkhäuser (2005).

[57] T. Roubíček, Thermodynamics of rate-independent processes in viscous solids at small strains. SIAM J. Math. Anal. 42 (2010) $256-297$.

[58] T. Roubíček, L. Scardia and C. Zanini, Quasistatic delamination problem. Contin. Mech. Thermodyn. 21 (2009) $223-235$.

[59] J. Simon, Compact sets in the space $L^{p}(0, T ; B)$. Ann. Mat. Pura Appl. (1987).

[60] M. Thomas, Griffith formula for mode-III-interface-cracks in strainhardening compounds. Mech. Adv. Mater. Struct. 12 (2008) $428-437$.

[61] M. Thomas, Rate-independent damage processes in nonlinearly elastic materials. Ph.D. thesis, Humboldt-Universität zu Berlin (2010).

[62] M. Thomas, Quasistatic damage evolution with spatial BV-regularization. Discrete Contin. Dyn. Syst. Ser. S 6 (2013) $235-255$.

[63] M. Thomas, Uniform Poincaré-Sobolev and relative isoperimetric inequalities for classes of domains. Accepted for publication in Discrete Contin. Dyn. Syst. WIAS-Preprint 1797 (2013).

[64] M. Thomas and A. Mielke, Damage of nonlinearly elastic materials at small strain: existence and regularity results. Zeit. angew. Math. Mech. 90 (2010) 88-112.

[65] M. Thomas and R. Rossi, Rate-independent Systems with viscosity and inertia: existence and evolutionary T-convergence. In preparation (2014). 